Part of Journal of Research of the National Bureau of Standards, Volume 28, April 1942

\title{
TENSILE AND COMPRESSIVE PROPERTIES OF SOME STAINLESS-STEEL SHEETS
}

\author{
By C. S. Aitchison, Walter Ramberg, L. B. Tuckerman, \\ and Herbert L. Whittemore
}

\section{ABSTRACT}

Tensile and compressive tests were made on specimens from chromium-nickel (17-7 and 18-7) stainless-steel sheets, with cold-reductions from zero percent (annealed) to 50 percent, and thicknesses from 0.01 to 0.06 in. The tensile yield strengths ranged from 34 to $200 \mathrm{kips} / \mathrm{in}^{2}$. The effect of a stress-relieving treatment at $300^{\circ} \mathrm{C}$ for 24 hours was investigated for one of the compositions.

The tensile tests were made on standard specimens. The compressive tests were made by the pack method developed at the National Bureau of Standards and by the cylinder method developed by Russell Franks, of the Union Carbide \& Carbon Research Laboratories. Tests were made on both longitudinal and transverse specimens from each sheet.

The results are given in tables and stress-strain curves to facilitate application in the design of light-weight structures from these materials. The effect of the degree of cold-reduction and of the stress-relieving treatment on the shape of the stress-strain curves and on the tensile and compressive properties is discussed.

\section{CONTENTS}

I. Introduction

II. Material

III. Method of testing

1. Tensile tests

2. Pack compressive tests

3 . Cylinder compressive tests.

IV. Results and discussion

V. Conclusions

VI. References

\section{INTRODUCTION}

There is a definite trend in structural design to use less material and so design the members that they are more efficient under load. Although this trend is noticeable in stationary structures, it is particularly marked in mobile structures, such as aircraft, where reduction in weight contributes directly to increased pay load, increased cruising range, and higher speed.

Stainless steel, available in sheets of a wide range of thickness, has, when cold-worked, a high strength-weight ratio, and since it is quite highly resistant to corrosion, little, if any, loss of strength results during its service life. 
To make the most efficient use of a material, particularly where thin sheets are employed, the designer must have accurate and reliable data on the tensile and compressive properties. Many aircraft members are subjected to high compressive stress-obviously compression members should not fail at loads below those required to produce tensile failure in some other part of the structure.

To provide data on the tensile and compressive properties of stainless-steel sheets, the following manufacturers sponsored an investigation at the National Bureau of Standards under the Research Associate Plan [1]: ${ }^{1}$
Allegheny Ludlum Steel Corporation.
American Rolling Mill Co.
American Steel \& Wire Co.
Carnegie-Illinois Steel Corporation.
Crucible Steel Company of America.
Electro Metallurgical Co.
International Nickel Co.
Republic Steel Corporation.
Rustless Iron \& Steel Corporation.

The members of the sponsors' committee representing the manufacturers were:

Milton Male, chairman, United States Steel Corporation.

L. S. Bergen, Crucible Steel Company of America.

V. B. Browne, Allegheny Ludlum Steel Corporation.

A. L. Feild, Rustless Iron \& Steel Corporation.

Russell Franks, Union Carbide \& Carbon Research Laboratories.

H. J. French, International Nickel Co.

T. F. Olt, American Rolling Mill Co.

E. C. Smith, Republic Steel Corporation.

The purpose of this investigation was to determine the tensile and compressive properties of stainless-steel sheets of two chemical compositions which are commercially available and particularly suitable for aircraft structures. Compressive tests were to be made on flat sheet by the pack method [2] developed at the National Bureau of Standards and on sheet rolled into cylinders by the cylinder method [3] developed by Franks and Binder.

\section{MATERIAL}

The stainless steel used in this investigation was obtained from two of the cooperating manufacturers. In this report the heat of steel from one manufacturer will be called " $17-7$ sheet" and the heat from the second manufacturer " $18-7$ sheet." The chemical compositions, given in table 1, were furnished by the manufacturers.

The sheets were cold-rolled to the nominal thickness after the final anneal in order to increase the mechanical properties. "Coldreduction" is defined as the percentage decrease in thickness after the final anneal. The thickness and the cold-reduction for the 17-7 sheets are given in table 2 and for the $18-7$ sheets in table 3 . For indentification, the sheets were numbered consecutively as they were received.

1 Numbers in brackets indicate the literature references at the end of this paper. 


\section{METHOD OF TESTING}

\section{TENSILE TESTS}

From each 17-7 sheet, two tensile specimens were taken longitudinally and two transversely to the direction in which the sheets had been rolled. One specimen of each was stress-relieved by heating to $300^{\circ} \mathrm{C}$ for 24 hours, followed by air-cooling. From each 18-7 sheet, one tensile specimen was taken longitudinally and one transversely. None of the specimens of the 18-7 sheet was stress-relieved. The specimens were ASTM standard test specimens [4], 1/2 in. wide and 2 -in. gage length. The cross-sectional area of each specimen was computed from its measured thickness and width.

The specimens were tested in a Baldwin-Southwark testing machine having a fluid-support, a Tate-Emery load-indicating mechanism, and scale ranges of 1,10 , and $20 \mathrm{kips}^{2}$ Templin grips were used to hold the specimens.

The strain was measured by a pair of Tuckerman 2-in. optical strain gages, having 0.4-in. knife-edges and lozenges. Each division of the vernier scale for these gages corresponded to a strain of 0.000004 . The readings were estimated to one-half a division. The strain gages were attached to opposite faces of the reduced section when the specimen was under an initial load, not exceeding $4.2 \mathrm{kips} / \mathrm{in}^{2}$.

For stresses up to the yield strength, the loading rate was about $\left(2 \mathrm{kips} / \mathrm{in}^{2}\right) / \mathrm{min}$. The speed of testing from yield strength to failure corresponded to a movement of the testing-machine head of 0.03 in./min. The value of initial strain, corresponding to the stress at the initial load, was obtained by a least-square extrapolation to zero stress.

Marks were spaced $1 / 4$ in. apart in the gage length, and the elongation was measured in accordance with AST Mspecifications [4].

\section{PACK COMPRESSIVE TESTS}

Pack compressive tests were made on both longitudinal and transverse specimens taken from all sheets having a thickness of 0.020 in. or greater. These tests were not made on the 0.010 -in. sheet nor on any sheets which had been stress-relieved. Each pack consisted of several specimens taken from the same sheet and in the same direction. The specimens for the packs from 0.020-, 0.035-, and 0.040-in. sheet were cemented together with fused shellac, but the specimens from the 0.060 -in. sheet were not cemented together. The fusing temperature was not higher than $66^{\circ} \mathrm{C}$ and the fusing time not more than 8 hours. The number of specimens and the lengths of the packs are given in tables 4 and 5 . There was a steel clamp at each end of each pack.

For the packs tested in the subpress (see tables), the middle specimen was about 0.03 in. wider than the others. For all the other packs, all the specimens were of the same width to facilitate machining.

The cross-sectional area of each pack was determined by the weight method [5]. The length was measured before and the weight and density determined after the pack was tested and the shellac removed.

${ }^{2} \mathrm{~A}$ kip is $1,000 \mathrm{lb}$. 
The packs were loaded in Baldwin-Southwark testing machines having fluid-supports, Tate-Emery load-indicating mechanisms, and scale ranges of 6,24 , and 120 , or 1,10 , and 20 kips. They were tested between hardened and ground steel blocks. To apply the load uniformly, a shim of plaster of paris was cast between the upper block and the head of the testing machine.

The strain was measured by a pair of 1-in. Tuckerman optical strain gages, having 0.2 in. knife-edges and lozenges. Each division of the vernier scale of these gages corresponded to a strain of 0.000004 . The readings were estimated to one-half a division. The strain gages were attached to the middle of the opposite edge faces when the pack was under the initial load, not exceeding $2.3 \mathrm{kip} / \mathrm{in}^{2}$.

The rate of loading was about $\left(2 \mathrm{kips} / \mathrm{in} .^{2}\right) / \mathrm{min}$. The value of strain corresponding to the stress at the initial load was obtained by a least-square extrapolation to zero stress.

\section{CYLINDER COMPRESSIVE TESTS}

The specimens for the cylinder compressive tests were taken both longitudinally and transversely from all sheets except those having a thickness of $0.060 \mathrm{in}$. The specimens were rolled to shape and the axial butt joints soldered under the direction of Russell Franks at the Union Carbide and Carbon Research Laboratories.

Additional longitudinal and transverse cylinders from 17-7 sheets were given a stress-relieving treatment, before they were soldered, by heating at $300^{\circ} \mathrm{C}$ for 24 hours followed by air-cooling.

The dimensions of the cylinders are given in tables 4 and 5 . The bases of the cylinders were plane and were parallel within 0.0004 in. The ends of the cylinders were restrained against crinkling by annular castings of Wood's metal having a thickness of about $1 / 4$ in. along the length of the cylinder. A clearance was maintained between the end face of the casting and the end face of the cylinder, to assure that the compressive load would act only on the cylinder. The cross-sectional area was determined by the weight method [5] after the specimens were machined but before they were rolled into cylinders.

The procedure for loading and measuring strain was the same for the cylinders as for the packs. The strain gages were attached to opposite elements of the outside cylindrical surface when the cylinder was under the initial load. Each of those elements was about an equal distance from the butt joint.

\section{RESULTS AND DISCUSSION}

The stress-strain curves for each specimen are shown in figures 1 to 55 . Figure 56 shows the individual points for the $18-7,0.060$-in. sheet having 47.7 percent cold-reduction (fig. 55 ) to indicate the order of agreement between the points and the curves drawn through them. The other stress-strain curves are drawn without indicating the individual points in order to facilitate the ease of obtaining tangent moduli. They are all plotted to the same scales, and a straight-line projection of these curves in all cases would pass through the origin at zero stress and zero strain. The slope of the straight line was determined from the low-load data by the method of least squares, 
which, although requiring a certain amount of computation, is less dependent upon judgment than is the drawing of a line through the points. The reason for obtaining this low-load (or initial) slope is that it permits the determination of the yield-strength value (offset $=0.2$ percent) for each specimen individually.

The tensile and compressive properties of the specimens are given in tables 4 and 5. For all sheets, except those that were stressrelieved, both the longitudinal and the transverse tensile strengths were significantly greater for the 18-7 stainless steel than for the 17-7 stainless steel with comparable cold-reduction. The elongations for the longitudinal specimens were consistently somewhat less for the 18-7 material than for the 17-7, but the elongations for the transverse specimens were nearly the same. There were no consistent differences between the yield strengths. In considering the elongations, it should be noted that the rates of loading are much lower than those customary in commercial practice and that, consequently, the elongations generally will be higher than those obtained commercially. For example, an increase in the rate of movement of the testing machine head from 0.03 to $0.10 \mathrm{in}$. $/ \mathrm{min}$ lowered the elongation of a representative sample from 32.0 to 15.5 percent.

Certain differences were found in the shape of the stress-strain curves, depending upon the orientation of the specimens in the sheet and the nature of the loading; that is, tension or compression. For the annealed sheets (zero cold-reduction), the tensile and pack compressive stress-strain curves were almost the same. The ordinates of each cylinder compressive stress-strain curve were in all cases larger than the corresponding ordinates of both the tensile and pack compressive stress-strain curves. This was shown by the cylinder compressive yield strengths, which exceeded the pack compressive yield strengths by more than 14 percent.

For the cold-reduced sheets, regardless of thickness, the tensile stress-strain curves for the longitudinal specimens and for the transverse specimens were significantly different in shape. However, the tensile yield strengths for the longitudinal specimens were about the same as for the transverse specimens. There were large differences in shape between the compressive stress-strain curves for the longitudinal packs, or cylinders, and for the transverse packs, or cylinders. These differences were greater with increased cold-reduction.

For the cold-reduced sheets, the ordinates of the compressive stress-strain curves for transverse packs, or cylinders, were significantly larger than the corresponding ordinates of the tensile curves for either the longitudinal or transverse specimens. However, the ordinates of the compressive stress-strain curves for the longitudinal packs, or cylinders, were markedly smaller than the corresponding ordinates of the tensile curves for either the longitudinal or the transverse specimens.

For the cold-reduced sheets, the compressive yield strengths for the transverse cylinders and for the transverse packs were nearly the same. However, the compressive yield strengths for the longitudinal cylinders exceeded those for the longitudinal packs by more than 7 percent.

The stress-strain curves for stress-relieved specimens from the coldreduced sheets were significantly different in shape from the curves from corresponding specimens which were not stress-relieved. The 
curves for the stress-relieved specimens did not change in slope as rapidly over the lower portion of the curve but, particularly for coldreductions up to 20 percent, changed more rapidly over the upper portion of the curve in the neighborhood of the yield strength.

The curves of strengths and elongations plotted against coldreductions (figs. 57 to 66 ) were not smooth, but they were remarkably regular considering that they were obtained from only one specimen for each cold-reduction. They suggest an approximate relationship between strength or elongation and cold-reduction independent of thickness. They show that the strengthening effect of cold-reduction is much greater on the yield strength than upon the tensile strength and that this effect is more pronounced in compression for transverse packs or cylinders than for longitudinal packs or cylinders.

For the 17-7 sheets, the tensile strengths for stress-relieved specimens were nearly the same as those for the specimens not stressrelieved. However, the elongations for the former were significantly greater than for the latter. For cold-reductions less than 20 percent, stress-relieving had very little effect on the tensile yield strength. For cold-reductions greater than 20 percent, stress-relieving significantly increased the tensile yield strengths. Stress-relieving caused no difference in compressive yield strength for transverse cylinders when the cold-reduction was small. At 20-percent coldreduction, however, the yield strength of stress-relieved transverse cylinders was significantly greater than that of the nonstress-relieved cylinders, and the difference increased with the degree of cold-reduction.

\section{CONCLUSIONS}

The stress-strain graphs and the tensile and compressive properties of 17-7 and 18-7 stainless steel sheets, with longitudinal tensile yield strengths from 34 to $200 \mathrm{kips} / \mathrm{in}^{2}{ }^{2}$ (corresponding to a range of sheets from annealed to 50-percent cold-reduction) and with thicknesses from 0.01 to 0.06 in., justify the following conclusions:

1. For annealed sheet, the stress-strain curves and the yield strengths from both tensile and pack compressive tests were about the same. The ordinates of the compressive stress-strain curves for either longitudinal or transverse cylinders were in all cases larger than the corresponding ordinates for tensile or pack compressive stressstrain curves.

2. For cold-reduced sheet, the tensile stress-strain curves for longitudinal specimens differed in shape from the tensile stress-strain curves for transverse specimens but the yield strengths for the longitudinal and transverse specimens were about the same.

3 . For cold-reduced sheet, the compressive stress-strain curves for the longitudinal packs, or cylinders, and for the transverse packs, or cylinders, differed widely in shape. The yield strengths for the longitudinal packs, or cylinders, were markedly less than those for the transverse packs, or cylinders.

4. For cold-reduced sheet, the ordinates of the compressive stressstrain curves for transverse packs, or cylinders, were significantly larger than the corresponding ordinates of the tensile stress-strain curves for either longitudinal or transverse specimens. But the ordinates of the compressive stress-strain curves for longitudinal packs, or cylinders, were appreciably smaller than the corresponding ordinates 
of the tensile stress-strain curves for either longitudinal or transverse specimens.

5. With comparable cold-reduction, the tensile strengths and the tensile and compressive yield strengths were about the same for all thicknesses of sheet investigated.

6. Stress-relieving increased the tensile and compressive yield strengths in both longitudinal and transverse directions. This effect was more marked the greater the amount of cold-reduction.

The authors express their appreciation to R. W. Gerby, G. E. Marquis, and W. H. Mather for their work in carrying out the tests and their helpful comments and suggestions.

\section{REFERENCES}

[1] Research associates at the Bureau of Standards, NBS Circular C296 (1926).

[2] C. S. Aitchison and L. B. Tuckerman, The "Pack" Method for Compressive Tests of Thin Specimens of Materials Used in Thin-Wall Structures, NACA Rep. 649 (1939).

[3] Russell Franks and W. O. Binder, The stress-strain characteristics of cold-rolled austenitic stainless steels in compression as determined by the cylinder test method, Proc. ASTM 41, 629 (1941).

[4] Tentative Methods of Tension Testing of Metallic Materials, E8-40T. ASTM Standards, 1940 supplement, pt. 1, Metals, p. 453.

[5] James A. Miller, Determination of cross-sectional areas of structural members, J. Research NBS 23, 329 (1939) RP1237.

TABLE 1.-Chemical compositions of sheet

[Analysis as determined by the producers]

\begin{tabular}{|c|c|c|}
\hline Elements & 17-7 sheet & 18-7 sheet \\
\hline $\begin{array}{l}\text { Carbon } \\
\text { Manganese } \\
\text { Phosphorus. } \\
\text { Sulfur } \\
\text { Silicon } \\
\text { Chromium } \\
\text { Nickel }\end{array}$ & $\begin{array}{r}\text { Percent } \\
0.12 \\
1.26 \\
0.015 \\
.009 \\
.36 \\
17.44 \\
7.18\end{array}$ & $\begin{array}{c}\text { Percent } \\
0.11 \\
.56 \\
.014 \\
.015 \\
.272 \\
17.90 \\
6.72\end{array}$ \\
\hline
\end{tabular}

TABLE 2.-Thickness and cold-reduction of $17-\gamma$ sheets

\begin{tabular}{|c|c|c|}
\hline Sheet number & $\begin{array}{c}\text { Thickness, } \\
\text { nominal }\end{array}$ & $\begin{array}{l}\text { Cold- } \\
\text { reduction } 1\end{array}$ \\
\hline $\begin{array}{l}225 \\
214 \\
219 \\
221 \\
223\end{array}$ & $\begin{array}{c}\text { Inch } \\
0.01 \\
.01 \\
.01 \\
.01 \\
.01\end{array}$ & $\begin{array}{r}\text { Percent } \\
0.0 \\
8.2 \\
18.0 \\
28.0 \\
37.0\end{array}$ \\
\hline $\begin{array}{l}218 \\
216 \\
204 \\
202 \\
200\end{array}$ & $\begin{array}{l}.02 \\
.02 \\
.02 \\
.02 \\
.02\end{array}$ & $\begin{array}{r}0.0 \\
8.2 \\
18.0 \\
30.5 \\
37.5\end{array}$ \\
\hline $\begin{array}{l}197 \\
209 \\
207 \\
205\end{array}$ & $\begin{array}{l}.04 \\
.04 \\
.04 \\
.04 \\
.04\end{array}$ & $\begin{array}{r}0.0 \\
9.2 \\
23.6 \\
28.5 \\
38.8\end{array}$ \\
\hline
\end{tabular}

1 Values reported by the producer. 
506 Journal of Research of the National Bureau of Standards

TABLE 3.-Thickness and cold-reduction of 18-7 sheet

\begin{tabular}{|c|c|c|c|c|c|}
\hline Sheet number & $\begin{array}{c}\text { Thickness, } \\
\text { nominal }\end{array}$ & $\begin{array}{l}\text { Cold- } \\
\text { reduction } 1\end{array}$ & Sheet number & $\begin{array}{c}\text { Thickness, } \\
\text { nominal }\end{array}$ & $\begin{array}{l}\text { Cold- } \\
\text { reduction } 1\end{array}$ \\
\hline $\begin{array}{l}51 \\
50 \\
52 \\
53\end{array}$ & $\begin{array}{c}\text { Inch } \\
0.01 \\
.01 \\
.01 \\
.01 \\
.01\end{array}$ & $\begin{array}{r}\text { Percent } \\
0.0 \\
12.0 \\
19.6 \\
31.5 \\
40.0\end{array}$ & $\begin{array}{l}36-125 \\
26- \\
27 \\
86\end{array}$ & $\begin{array}{c}\text { Inch } \\
0.035 \\
.035 \\
.035 \\
.035 \\
.035\end{array}$ & $\begin{array}{r}\text { Percent } \\
0.0 \\
7.5 \\
7.5 \\
19.6 \\
29.2\end{array}$ \\
\hline $\begin{array}{l}59 \\
55 \\
58 \\
68 \\
58\end{array}$ & $\begin{array}{l}.01 \\
.01 \\
.02 \\
.02 \\
.02\end{array}$ & $\begin{array}{r}50.0 \\
52.5 \\
0.0 \\
.0 \\
5.3\end{array}$ & $\begin{array}{l}37 \\
38 \\
39 \\
41 \\
7\end{array}$ & $\begin{array}{l}.035 \\
.035 \\
.035 \\
.06 \\
.06\end{array}$ & $\begin{array}{r}39.7 \\
47.0 \\
47.0 \\
0.0 \\
6.7\end{array}$ \\
\hline $\begin{array}{l}69 \\
445 \\
46 \\
47\end{array}$ & $\begin{array}{l}.02 \\
.02 \\
.02 \\
.02 \\
.02\end{array}$ & $\begin{array}{r}5.3 \\
18.3 \\
18.3 \\
29.8 \\
29.8\end{array}$ & 8 & $\begin{array}{l}.06 \\
.06 \\
.06 \\
.06\end{array}$ & $\begin{array}{l}18.1 \\
30.5 \\
36.9 \\
47.7\end{array}$ \\
\hline $\begin{array}{l}56 \\
66 \\
49 \\
45\end{array}$ & $\begin{array}{l}.02 \\
.02 \\
.02 \\
.02 \\
.035\end{array}$ & $\begin{array}{r}37.5 \\
37.5 \\
50.0 \\
50.0 \\
0.0\end{array}$ & & . & \\
\hline
\end{tabular}

1 Values reported by the producer. 
TABLE 4.-Results of tensile and compressive tests on 17-7 stainless stee

\begin{tabular}{|c|c|c|c|c|c|c|c|c|c|c|c|c|c|c|c|c|}
\hline \multirow[b]{2}{*}{$\begin{array}{l}\text { Cold- } \\
\text { reduc- } \\
\text { tion }\end{array}$} & \multicolumn{6}{|c|}{ Tension } & \multicolumn{5}{|c|}{ Pack compression } & \multicolumn{5}{|c|}{ Cylinder compression } \\
\hline & $\begin{array}{l}\text { Specimen } \\
\text { number }\end{array}$ & $\begin{array}{c}\text { Slope of } \\
\text { straight } \\
\text { line } 1\end{array}$ & $\begin{array}{c}\text { Yield } \\
\text { strength;2 } \\
\text { offset }= \\
0.2 \%\end{array}$ & $\begin{array}{c}\text { Tensile } \\
\text { strength }\end{array}$ & $\begin{array}{l}\text { Elonga- } \\
\text { tion in } \\
2 \text { in. }\end{array}$ & Remarks $^{3}$ & Pack number & Length & $\begin{array}{l}\text { Num- } \\
\text { ber of } \\
\text { speci- } \\
\text { mens } \\
\text { in pack }\end{array}$ & $\begin{array}{l}\text { Slope of } \\
\text { straight } \\
\text { line } 1\end{array}$ & $\begin{array}{c}\text { Yield } \\
\text { strength;2 } \\
\text { offset= } \\
0.2 \%\end{array}$ & $\begin{array}{l}\text { Cylinder } \\
\text { number }\end{array}$ & Length & $\begin{array}{c}\text { Out- } \\
\text { side di- } \\
\text { ameter, } \\
\text { nom- } \\
\text { inal }\end{array}$ & $\begin{array}{l}\text { Slope of } \\
\text { straight } \\
\text { line 1 }\end{array}$ & $\begin{array}{c}\text { Yield } \\
\text { strength; } \\
\text { offset }= \\
0.2 \%\end{array}$ \\
\hline \multicolumn{17}{|c|}{0.010 IN. THICK, AS-ROLLED, LONGITUDINAL } \\
\hline $\begin{array}{r}\text { Percent } \\
0.0 \\
8.2 \\
18.0 \\
28.0 \\
37.0\end{array}$ & $\begin{array}{l}225-\mathrm{B} 1 \mathrm{~L} \\
214-\mathrm{B} 1 \mathrm{~L} \\
219-\mathrm{B} 2 \mathrm{~L} \\
221-\mathrm{B} 1 \mathrm{~L} \\
223-\mathrm{B} 2 \mathrm{~L}\end{array}$ & $\begin{array}{r}\text { Kips/in. } .^{2} \\
29,100 \\
27,400 \\
28,500 \\
27,300 \\
26,500\end{array}$ & \begin{tabular}{|r|} 
Kips/in. \\
39.0 \\
77.9 \\
103.8 \\
133.1 \\
150.3
\end{tabular} & $\begin{array}{r}\text { Kips/in. } .^{2} \\
136.3 \\
155.6 \\
167.3 \\
174.7 \\
187.5\end{array}$ & $\begin{array}{l}\% \\
72.0 \\
60.0 \\
49.0 \\
46.0 \\
40.0\end{array}$ & $\begin{array}{l}\mathrm{d}(71.0) \\
\mathrm{d}(58.0) \\
\mathrm{b} \\
\mathrm{b} \\
\mathrm{c}\end{array}$ & $\begin{array}{c}\text { None_- } \\
\text { do } \\
\text { do }\end{array}$ & \begin{tabular}{|c|} 
Inches \\
\end{tabular} & $\mid \begin{array}{l} \\
\\
\end{array}$ & Kips/in. ${ }^{2}$ & Kips/in. ${ }^{2}$ & $\begin{array}{l}\text { 225-CX3L } \\
214-C X 3 L \\
219-C X 3 L \\
221-C X 3 L \\
223-C X 3 L\end{array}$ & $\begin{array}{r}\text { Inches } \\
1.749 \\
1,744 \\
1,750 \\
1.745 \\
1.753\end{array}$ & $\begin{array}{r}\text { Inches } \\
0.40 \\
.40 \\
.40 \\
.40 \\
.40\end{array}$ & \begin{tabular}{|c} 
Kips/in. \\
28,900 \\
26,100 \\
27,700 \\
25,700 \\
26,400
\end{tabular} & $\begin{array}{r}\text { Kips/in. }{ }^{2} \\
43.7 \\
60.5 \\
73.7 \\
90.2 \\
98.3\end{array}$ \\
\hline \multicolumn{17}{|c|}{ 0.010 IN. THICK, AS-ROLLED, TRANSVERSE } \\
\hline $\begin{array}{r}0.0 \\
8.2 \\
18.0 \\
28.0 \\
37.0\end{array}$ & $\begin{array}{l}\text { 225-B1T } \\
214-\mathrm{B} 1 \mathrm{~T} \\
219-\mathrm{B} 1 \mathrm{~T} \\
221-\mathrm{B} 1 \mathrm{~T} \\
223-\mathrm{B} 1 \mathrm{~T}\end{array}$ & $\begin{array}{l}29,100 \\
28,500 \\
28,400 \\
29,000 \\
28,900\end{array}$ & $\begin{array}{r}38.5 \\
76.7 \\
100.8 \\
121.0 \\
138.8\end{array}$ & $\begin{array}{l}120.9 \\
152.7 \\
161.9 \\
176.9 \\
186.4\end{array}$ & $\begin{array}{l}53.0 \\
53.5 \\
40.5 \\
42.0 \\
39.0\end{array}$ & $\begin{array}{l}\mathrm{b} \\
\mathrm{b} \\
\mathrm{b} \\
\mathrm{c} \\
\mathrm{d}(35.5)\end{array}$ & \begin{tabular}{|l} 
None \\
\\
\end{tabular} & 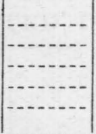 & - & 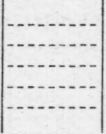 & 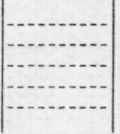 & $\begin{array}{l}225-\mathrm{CX} 3 T \\
214-\mathrm{CX} 3 T \\
219-\mathrm{CX} 3 T \\
221-\mathrm{CX} 3 T \\
223-\mathrm{CX} 3 \mathrm{~T}\end{array}$ & $\begin{array}{l}\text { 1. } 746 \\
\text { 1. } 750 \\
1.752 \\
1.740 \\
1.746\end{array}$ & $\begin{array}{l}0.40 \\
.40 \\
.40 \\
.40 \\
.40\end{array}$ & $\begin{array}{l}33,200 \\
30,000 \\
30,500 \\
29,400 \\
28,600\end{array}$ & $\begin{array}{r}42.6 \\
81.5 \\
104.4 \\
137.8 \\
150.0\end{array}$ \\
\hline \multicolumn{17}{|c|}{ 0.010 IN. THICK, STRESS-RELIEVED, LONGITUDINAL } \\
\hline $\begin{array}{r}0.0 \\
8.2 \\
18.0 \\
28.0 \\
37.0\end{array}$ & $\begin{array}{l}225-Y 3 L \\
214-Y 3 L \\
219-Y 4 L \\
221-Y 3 L \\
223-Y 4 L\end{array}$ & $\begin{array}{l}30,300 \\
28,800 \\
28,400 \\
27,900 \\
29,400\end{array}$ & $\begin{array}{r}39.2 \\
78.2 \\
108.4 \\
141.5 \\
171.7\end{array}$ & $\begin{array}{l}135.5 \\
149.7 \\
162.9 \\
174.4 \\
198.2\end{array}$ & $\begin{array}{l}84.5 \\
67.0 \\
54.0 \\
53.0 \\
37.0\end{array}$ & $\begin{array}{l}\mathrm{c} \\
\mathrm{c} \\
\mathrm{c} \\
\mathrm{b} \\
\mathrm{b}\end{array}$ & \begin{tabular}{|c|} 
None \\
\\
\end{tabular} & $\mid \begin{array}{l}{[-\ldots . .} \\
\cdots\end{array}$ & \begin{tabular}{|c|} 
\\
\hdashline
\end{tabular} & $\mid \begin{array}{l} \\
\end{array}$ & \begin{tabular}{|c|} 
\\
\end{tabular} & $\begin{array}{l}\text { 225-CX4L } \\
214-C X 4 L \\
219-C X 4 L \\
221-C X 4 L \\
223-C X 4 L\end{array}$ & $\begin{array}{l}1.749 \\
1.744 \\
\text { 1. } 747 \\
1.750 \\
1.749\end{array}$ & $\begin{array}{l}0.40 \\
.40 \\
.40 \\
.40 \\
.40\end{array}$ & $\begin{array}{l}29,700 \\
28,100 \\
31,500 \\
28,600 \\
29,200\end{array}$ & $\begin{array}{r}43.5 \\
71.6 \\
89.7 \\
109.7 \\
123.4\end{array}$ \\
\hline
\end{tabular}

1 Obtai ned by least-square fit to lower portion of stress-strain graph.

3 Letters describe location of fracture in accordance with section 24 and figure 19, page 462 of ASTM Specification E8-40T. Numbers in parentheses are alternate elongation values determined from sum of the distances $A$ to $F$ and $B$ to $E$ shown in that figure. 
TABLE 4.-Results of tensile and compressive tests on 17-7 stainless steel-Continued

\begin{tabular}{|c|c|c|c|c|c|c|c|c|c|c|c|c|c|c|c|c|}
\hline \multirow[b]{2}{*}{$\begin{array}{l}\text { Cold- } \\
\text { reduc- } \\
\text { tion }\end{array}$} & \multicolumn{6}{|c|}{ Tension } & \multicolumn{5}{|c|}{ Pack compression } & \multicolumn{5}{|c|}{ Cylinder compression } \\
\hline & $\begin{array}{l}\text { Specimen } \\
\text { number }\end{array}$ & $\begin{array}{l}\text { Slope of } \\
\text { straight } \\
\text { line }^{1}\end{array}$ & $\begin{array}{c}\text { Yield } \\
\text { strength; }{ }^{2} \\
\text { offiset }= \\
0.2 \%\end{array}$ & $\begin{array}{l}\text { Tensile } \\
\text { strength }\end{array}$ & $\begin{array}{c}\text { Elonga- } \\
\text { tion in } \\
2 \text { in. }\end{array}$ & Remarks $^{3}$ & Pack number & Length & $\begin{array}{c}\text { Num- } \\
\text { ber of } \\
\text { speci- } \\
\text { mens } \\
\text { in pack }\end{array}$ & $\begin{array}{c}\text { Slope of } \\
\text { straight } \\
\text { line }^{1}\end{array}$ & $\begin{array}{c}\text { Yield } \\
\text { strength; } \\
\text { offset= } \\
0.2 \%\end{array}$ & $\begin{array}{l}\text { Cylinder } \\
\text { number }\end{array}$ & Length & $\begin{array}{c}\text { Out- } \\
\text { side di- } \\
\text { ameter, } \\
\text { nom- } \\
\text { inal }\end{array}$ & $\begin{array}{c}\text { Slope of } \\
\text { straight } \\
\text { line }^{1}\end{array}$ & $\begin{array}{c}\text { Yield } \\
\text { strength; } 2 \\
\text { offset }= \\
0.2 \%\end{array}$ \\
\hline \multicolumn{17}{|c|}{ 0.010 IN. THICK, STRESS-RELIEVED, TRANSVERSE } \\
\hline $\begin{array}{r}\text { Percent } \\
0.0 \\
8.2 \\
18.0 \\
28.0 \\
37.0\end{array}$ & $\begin{array}{l}225-Y 3 T \\
214-Y 3 T \\
219-Y 3 T \\
221-Y 4 T \\
223-Y 3 T\end{array}$ & $\begin{array}{r}\text { Kips/in. } \\
29,400 \\
28,000 \\
29,000 \\
28,800 \\
29,600\end{array}$ & $\begin{array}{r}\text { Kips/in. } \\
39.0 \\
77.7 \\
109.5 \\
139.8 \\
156.5\end{array}$ & $\begin{array}{r}\text { Kips/in. } \\
135.9 \\
148.6 \\
161.0 \\
176.6 \\
189.2\end{array}$ & $\begin{array}{l}\% \\
81.5 \\
67.5 \\
55.0 \\
58.0 \\
41.5\end{array}$ & $\begin{array}{l}c \\
b \\
b \\
c \\
c\end{array}$ & $\begin{array}{l}\text { None } \\
\text { do } \\
\end{array}$ & \begin{tabular}{|c|} 
Inches \\
\end{tabular} & \begin{tabular}{|c|} 
\\
\hdashline$\ldots$ \\
\end{tabular} & \begin{tabular}{|l} 
Kips/in. \\
\end{tabular} & \begin{tabular}{|c|} 
Kips/in. \\
\end{tabular} & $\begin{array}{l}225-\mathrm{CX} 4 \mathrm{~T} \\
214-\mathrm{CX} 4 \mathrm{~T} \\
219-\mathrm{CX} 4 \mathrm{~T} \\
221-\mathrm{CX} 4 \mathrm{~T} \\
223-\mathrm{CX} 4 \mathrm{~T}\end{array}$ & $\begin{array}{r}\text { Inches } \\
1.750 \\
1.750 \\
1.750 \\
1.749 \\
1.754\end{array}$ & $\begin{array}{r}\text { Inches } \\
0.40 \\
.40 \\
.40 \\
.40 \\
.40\end{array}$ & $\begin{array}{r}\text { Kips/in. } \\
26,900 \\
26,600 \\
29,400 \\
29,900 \\
31,300\end{array}$ & $\begin{array}{r}\text { Kips/in. } \\
42.9 \\
80.3 \\
112.7 \\
146.6 \\
170.4\end{array}$ \\
\hline \multicolumn{17}{|c|}{ 0.020 IN. THICK, AS-ROLLED, LONGITUDINAL } \\
\hline $\begin{array}{r}0.0 \\
8.2 \\
18.0 \\
30.5 \\
37.5\end{array}$ & $\begin{array}{l}218-\mathrm{B} 2 \mathrm{~L} \\
216-\mathrm{B} 2 \mathrm{~L} \\
204-\mathrm{B} 2 \mathrm{~L} \\
202-\mathrm{B} 2 \mathrm{~L} \\
200-\mathrm{B} 2 \mathrm{~L}\end{array}$ & $\begin{array}{l}29,000 \\
28,600 \\
27,600 \\
27,800 \\
26,700\end{array}$ & $\begin{array}{r}36.7 \\
78.3 \\
111.0 \\
129.8 \\
151.9\end{array}$ & $\begin{array}{l}136.4 \\
158.0 \\
167.7 \\
175.7 \\
185.3\end{array}$ & $\begin{array}{l}77.0 \\
55.0 \\
43.5 \\
47.0 \\
37.0\end{array}$ & $\begin{array}{l}\mathrm{b} \\
\mathrm{b} \\
\mathrm{b} \\
\mathrm{b} \\
\mathrm{b}\end{array}$ & $\begin{array}{l}\text { 218-CN2L } \\
216-C N 1 L \\
204-C N 1 L \\
202-C N 1 L \\
200-C N 1 L \\
\end{array}$ & $\begin{array}{l}2.502 \\
2.501 \\
2.501 \\
2.501 \\
2.501\end{array}$ & $\begin{array}{l}21 \\
21 \\
21 \\
21 \\
21\end{array}$ & $\begin{array}{l}27,400 \\
28,400 \\
28,600 \\
28,200 \\
27,600\end{array}$ & $\begin{array}{l}37.9 \\
49.4 \\
67.2 \\
75.4 \\
83.8\end{array}$ & $\begin{array}{l}\text { 218-CX1L } \\
216-C X 3 L \\
204-C X 1 L \\
202-C X 1 L \\
200-C X 1 L\end{array}$ & $\begin{array}{l}1.998 \\
1.995 \\
1.997 \\
1.998 \\
1.998\end{array}$ & $\begin{array}{r}0.80 \\
.80 \\
.80 \\
.80 \\
.80\end{array}$ & $\begin{array}{l}32,400 \\
27,700 \\
28,700 \\
26,800 \\
27,900\end{array}$ & $\begin{array}{l}43.3 \\
64.0 \\
77.7 \\
90.6 \\
96.7\end{array}$ \\
\hline \multicolumn{17}{|c|}{0.020 IN. THICK, AS-ROLLED, TRANSVERSE } \\
\hline $\begin{array}{r}0.0 \\
8.2 \\
18.0 \\
30.5 \\
37.5\end{array}$ & $\begin{array}{l}218-\mathrm{B} 2 \mathrm{~T} \\
216-\mathrm{B} 2 \mathrm{~T} \\
204-\mathrm{B} 2 \mathrm{~T} \\
202-\mathrm{B} 2 \mathrm{~T} \\
200-\mathrm{B} 2 \mathrm{~T}\end{array}$ & $\begin{array}{l}29,000 \\
28,500 \\
28,000 \\
28,500 \\
29,300\end{array}$ & $\begin{array}{r}36.7 \\
84.0 \\
106.9 \\
123.9 \\
143.0\end{array}$ & $\begin{array}{l}134.9 \\
153.8 \\
167.0 \\
179.4 \\
188.2\end{array}$ & $\begin{array}{l}76.0 \\
45.0 \\
54.0 \\
38.0 \\
35.5\end{array}$ & $\begin{array}{l}\mathrm{d}(73.5) \\
\mathrm{d}(43.5) \\
\mathrm{b} \\
\mathrm{b} \\
\mathrm{b}\end{array}$ & $\begin{array}{l}\text { 218-CN1T } \\
\text { 216-CN1T } \\
\text { 204-CN1T } \\
\text { 202-CP3T } 4 \\
200-\mathrm{CP} 2 \mathrm{~T} 4 \\
\end{array}$ & $\begin{array}{l}\text { 2. } 500 \\
2.501 \\
2.501 \\
2.497 \\
\text { 2. } 501\end{array}$ & $\begin{array}{r}21 \\
21 \\
21 \\
9 \\
9\end{array}$ & $\begin{array}{l}28,500 \\
29,200 \\
28,600 \\
28,900 \\
29,800\end{array}$ & $\begin{array}{r}37.5 \\
91.1 \\
117.3 \\
140.0 \\
165.0\end{array}$ & $\begin{array}{l}218-C X 3 T \\
216-C X 3 T \\
204-C X 1 T \\
202-C X 1 T \\
200-C X 1 T \\
\end{array}$ & $\begin{array}{l}2.000 \\
2.000 \\
1.997 \\
\text { 1. } 999 \\
\text { 2. } 000\end{array}$ & $\begin{array}{l}0.80 \\
.80 \\
.80 \\
.80 \\
.80\end{array}$ & $\begin{array}{l}31,800 \\
28,600 \\
28,100 \\
28,900 \\
28,800\end{array}$ & $\begin{array}{r}42.4 \\
88.9 \\
114.8 \\
139.2 \\
165.0\end{array}$ \\
\hline \multicolumn{17}{|c|}{0.020 IN. THICK, STRESS-RELIEVED, LONGITUDINAL } \\
\hline $\begin{array}{r}0.0 \\
8.2 \\
18.0 \\
30.5 \\
37.5\end{array}$ & $\begin{array}{l}218-Y 4 L \\
216-Y 4 L \\
204-Y 3 L \\
202-Y 3 L \\
200-Y 4 L\end{array}$ & $\begin{array}{l}29.100 \\
28,100 \\
28,200 \\
27,300 \\
27,000\end{array}$ & $\begin{array}{r}36.2 \\
80.4 \\
116.4 \\
140.0 \\
160.4\end{array}$ & $\begin{array}{l}133.3 \\
153.8 \\
163.3 \\
174.0 \\
180.8\end{array}$ & $\begin{array}{l}85.5 \\
62.5 \\
52.0 \\
47.0 \\
39.5\end{array}$ & $\begin{array}{l}c \\
b \\
b \\
c \\
b\end{array}$ & $\begin{array}{c}\text { None } \\
\\
\\
\\
\end{array}$ & $\cdots$ & & & & $\begin{array}{l}218-\mathrm{CX} 2 \mathrm{~L} \\
216-\mathrm{CX} 2 \mathrm{~L} \\
204-\mathrm{CX} 2 \mathrm{~L} \\
202-\mathrm{CX} 2 \mathrm{~L} \\
200-\mathrm{CX} 4 \mathrm{~L}\end{array}$ & $\begin{array}{l}1.996 \\
2.002 \\
2.002 \\
2.002 \\
2.001\end{array}$ & $\begin{array}{r}0.80 \\
.80 \\
.80 \\
.80 \\
.80\end{array}$ & $\begin{array}{l}32,800 \\
28,900 \\
31,100 \\
29,000 \\
27,400\end{array}$ & $\begin{array}{r}41.7 \\
77.5 \\
98.0 \\
113.0 \\
134.5\end{array}$ \\
\hline
\end{tabular}


0.020 IN. THICK, STRESS-RELIEVED, TRANSVERSE

\begin{tabular}{|c|c|c|c|c|c|c|c|c|c|c|c|c|c|c|c|c|}
\hline $\begin{array}{r}0.0 \\
8.2 \\
18.0 \\
30.5 \\
37.5\end{array}$ & $\begin{array}{l}\text { 218-Y4T } \\
216-Y 4 T \\
204-Y 3 T \\
202-Y 3 T \\
200-Y 4 T\end{array}$ & $\begin{array}{l}28,400 \\
28,800 \\
28,200 \\
28,800 \\
29,200\end{array}$ & $\begin{array}{r}36.0 \\
83.1 \\
112.1 \\
136.0 \\
158.6\end{array}$ & $\begin{array}{l}136.2 \\
148.0 \\
155.8 \\
172.0 \\
187.9\end{array}$ & $\begin{array}{l}86.5 \\
69.0 \\
62.0 \\
46.5 \\
36.5\end{array}$ & $\begin{array}{l}\mathrm{b} \\
\mathrm{c} \\
\mathrm{b} \\
\mathrm{b} \\
\mathrm{c}\end{array}$ & $\begin{array}{c}\text { None } \\
\quad \text { do } \\
\\
\\
\end{array}$ & - & - & ( & 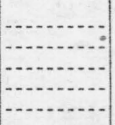 & $\begin{array}{l}\text { 218-CX2T } \\
216-\mathrm{CX} 2 \mathrm{~T} \\
204-\mathrm{CX} 2 \mathrm{~T} \\
202-\mathrm{CX} 2 \mathrm{~T} \\
200-\mathrm{CX} 3 \mathrm{~T}\end{array}$ & $\begin{array}{l}2.000 \\
2.000 \\
1.977 \\
1.961 \\
1.993\end{array}$ & $\begin{array}{l}0.80 \\
.80 \\
.80 \\
.80 \\
.80\end{array}$ & $\begin{array}{l}35,600 \\
29,000 \\
29,800 \\
29,600 \\
29,400\end{array}$ & $\begin{array}{r}42.0 \\
86.8 \\
117.8 \\
146.7 \\
176.4\end{array}$ \\
\hline \multicolumn{17}{|c|}{ 0.040 IN. THICK, AS-ROLLED, LONGITUDINAL } \\
\hline $\begin{array}{r}0.0 \\
9.2 \\
23.6 \\
28.5 \\
38.8\end{array}$ & $\begin{array}{l}\text { 197-B1L } \\
\text { 211-B1L } \\
\text { 209-B1L } \\
\text { 207-B1L } \\
\text { 205-B1L }\end{array}$ & $\begin{array}{l}29,900 \\
28,700 \\
27,600 \\
26,500 \\
26,200\end{array}$ & $\begin{array}{r}37.7 \\
83.9 \\
113.1 \\
135.5 \\
156.7\end{array}$ & $\begin{array}{l}127.7 \\
160.0 \\
162.0 \\
182.2 \\
191.4\end{array}$ & $\begin{array}{l}87.0 \\
61.0 \\
49.0 \\
45.0 \\
32.0\end{array}$ & $\begin{array}{l}\mathrm{c} \\
\mathrm{c} \\
\mathrm{c} \\
\mathrm{d}(42.0) \\
\mathrm{c}\end{array}$ & $\begin{array}{l}\text { 197-CN1L } \\
\text { 211-CN1L } \\
\text { 209-CN1L-..... } \\
\text { 207-CN1L } \\
205-C N 1 L\end{array}$ & $\begin{array}{l}2.501 \\
2.503 \\
2.491 \\
2.500 \\
2.501\end{array}$ & $\begin{array}{l}9 \\
9 \\
9 \\
9 \\
9\end{array}$ & $\begin{array}{l}30,400 \\
29,500 \\
28,400 \\
27,800 \\
26,700\end{array}$ & $\begin{array}{l}38.8 \\
59.1 \\
72.3 \\
83.1 \\
88.9\end{array}$ & $\begin{array}{l}\text { 197-CX1L } \\
\text { 211-CX1L } \\
\text { 209-CX1L } \\
\text { 207-CX1L } \\
\text { 205-CX1L }\end{array}$ & $\begin{array}{l}1.999 \\
1.993 \\
2.002 \\
2.002 \\
2.000\end{array}$ & $\begin{array}{l}1.53 \\
1.53 \\
1.53 \\
1.53 \\
1.53\end{array}$ & $\begin{array}{l}30,100 \\
31,200 \\
29,200 \\
28,500 \\
26,800\end{array}$ & $\begin{array}{r}45.0 \\
68.6 \\
83.3 \\
94.8 \\
107.3\end{array}$ \\
\hline \multicolumn{17}{|c|}{0.040 IN. THICK, AS-ROLLED, TRANSVERSE } \\
\hline $\begin{array}{r}0.0 \\
9.2 \\
23.6 \\
28.5 \\
38.8\end{array}$ & $\begin{array}{l}\text { 197-B1T } \\
211-\mathrm{B} 1 \mathrm{~T} \\
209-\mathrm{B} 1 \mathrm{~T} \\
207-\mathrm{B} 1 \mathrm{~T} \\
205-\mathrm{B} 2 \mathrm{~T}\end{array}$ & $\begin{array}{l}27,800 \\
27,500 \\
27,800 \\
28,200 \\
29,200\end{array}$ & $\begin{array}{r}37.9 \\
89.2 \\
110.6 \\
129.3 \\
156.6\end{array}$ & $\begin{array}{l}133.1 \\
159.6 \\
166.7 \\
180.8 \\
197.3\end{array}$ & $\begin{array}{l}82.5 \\
61.0 \\
51.0 \\
35.0 \\
21.0\end{array}$ & $\begin{array}{l}b \\
b \\
b \\
c \\
b\end{array}$ & $\begin{array}{l}\text { 197-CN1T } \\
\text { 211-CN1T-..- } \\
\text { 209-CN1T-...- } \\
\text { 207-CN1T } \\
\text { 205-CN1T.... }\end{array}$ & $\begin{array}{l}2.500 \\
2.502 \\
2.502 \\
2.501 \\
2.502\end{array}$ & $\begin{array}{l}9 \\
9 \\
9 \\
9 \\
9\end{array}$ & $\begin{array}{l}29,400 \\
28,300 \\
28,800 \\
29,800 \\
30,200\end{array}$ & $\begin{array}{r}38.5 \\
96.1 \\
122.7 \\
144.5 \\
175.1\end{array}$ & $\begin{array}{l}\text { 197-CX1T } \\
\text { 211-CX1T } \\
\text { 209-CX1T } \\
\text { 207-CX1T } \\
\text { 205-CX1T }\end{array}$ & $\begin{array}{l}2.002 \\
1.998 \\
1.993 \\
1.974 \\
2.001\end{array}$ & $\begin{array}{l}\text { 1. } 53 \\
\text { 1. } 53 \\
1.53 \\
1.53 \\
1.53\end{array}$ & $\begin{array}{l}27,900 \\
29,800 \\
29,600 \\
28,900 \\
30,500\end{array}$ & $\begin{array}{r}44.1 \\
94.1 \\
120.9 \\
144.4 \\
174.9\end{array}$ \\
\hline \multicolumn{17}{|c|}{ 0.040 IN. THICK, STRESS-RELIEVED, LONGITUDINAL } \\
\hline $\begin{array}{r}0.0 \\
9.2 \\
23.6 \\
28.5 \\
38.8\end{array}$ & $\begin{array}{l}\text { 197-Y4L } \\
211-Y 3 L \\
209-Y 3 L \\
207-Y 3 L \\
205-Y 3 L\end{array}$ & $\begin{array}{l}30,200 \\
28,700 \\
28,900 \\
27,000 \\
28,100\end{array}$ & $\begin{array}{r}38.1 \\
88.3 \\
121.3 \\
145.6 \\
170.0\end{array}$ & $\begin{array}{l}132.3 \\
153.4 \\
160.1 \\
175.5 \\
201.8\end{array}$ & $\begin{array}{l}87.0 \\
64.5 \\
55.5 \\
44.5 \\
18.0\end{array}$ & $\begin{array}{l}d(84.5) \\
b \\
b \\
b \\
b\end{array}$ & \begin{tabular}{|l|} 
None \\
do do \\
\end{tabular} & & & & & $\begin{array}{l}\text { 197-CX2L } \\
\text { 211-CX2L } \\
\text { 209-CX2L } \\
\text { 207-CX2L } \\
205-C X 2 L\end{array}$ & $\begin{array}{l}\text { 2. } 002 \\
2.000 \\
2.000 \\
2.000 \\
2.000\end{array}$ & $\begin{array}{l}\text { 1. } 53 \\
1.53 \\
1.53 \\
\text { 1. } 53 \\
1.53\end{array}$ & $\begin{array}{l}30,700 \\
31,200 \\
29,000 \\
29,100 \\
27,600\end{array}$ & $\begin{array}{r}43.8 \\
83.7 \\
104.5 \\
121.7 \\
145.5\end{array}$ \\
\hline \multicolumn{17}{|c|}{ 0.040 IN. THICK, STRESS-RELIEVED, TRANSVERSE } \\
\hline $\begin{array}{r}0.0 \\
9.2 \\
23.6 \\
28.5 \\
38.8\end{array}$ & $\begin{array}{l}197-Y 4 T \\
211-Y 4 T \\
209-Y 4 T \\
207-Y 3 T \\
205-Y 3 T\end{array}$ & $\begin{array}{l}28,600 \\
28,200 \\
28,700 \\
29,200 \\
30,100\end{array}$ & $\begin{array}{r}37.5 \\
90.3 \\
118.1 \\
139.2 \\
171.2\end{array}$ & $\begin{array}{l}127.5 \\
153.2 \\
158.9 \\
173.6 \\
206.4\end{array}$ & $\begin{array}{r}82.5 \\
65.5 \\
63.0 \\
38.5 \\
9.5\end{array}$ & $\begin{array}{l}\mathrm{c} \\
\mathrm{c} \\
\mathrm{c} \\
\mathrm{b} \\
\mathrm{b}\end{array}$ & $\begin{array}{c}\text { None } \\
\text { do..... } \\
\text { do } \\
\\
\end{array}$ & & & & & $\begin{array}{l}\text { 197-CX2T } \\
\text { 211-CX2T } \\
\text { 209-CX2T } \\
207-C X 2 T \\
\text { 205-CX2T }\end{array}$ & $\begin{array}{l}1.997 \\
2.000 \\
1.997 \\
1.999 \\
1.994\end{array}$ & $\begin{array}{l}\text { 1. } 53 \\
\text { 1. } 53 \\
1.53 \\
1.53 \\
1.53\end{array}$ & $\begin{array}{l}29,100 \\
29,000 \\
29,800 \\
32,900 \\
29,900\end{array}$ & $\begin{array}{r}43.4 \\
92.6 \\
124.6 \\
160.4 \\
193.5\end{array}$ \\
\hline
\end{tabular}

123 See footnotes 1,2 , and 3 , p. 507 .
One-half-inch-wide pack tested in subpress, lateral support, 39 pins on each side, $3 / 16$-in. spacing. 
TABLE 5.-Results of tensile and compressive tests on 18-7 stainless steel

\begin{tabular}{|c|c|c|c|c|c|c|c|c|c|c|c|c|c|c|c|c|}
\hline \multirow[b]{2}{*}{$\begin{array}{l}\text { Cold- } \\
\text { reduc- } \\
\text { tion }\end{array}$} & \multicolumn{6}{|c|}{ Tension } & \multicolumn{5}{|c|}{ Pack compression } & \multicolumn{5}{|c|}{ Cylinder compression } \\
\hline & $\begin{array}{l}\text { Specimen } \\
\text { number }\end{array}$ & $\begin{array}{l}\text { Slope of } \\
\text { straight } \\
\text { line }^{1}\end{array}$ & $\begin{array}{c}\text { Yield } \\
\text { strength; } \\
\text { offset } \\
=0.2 \%\end{array}$ & $\begin{array}{l}\text { Tensile } \\
\text { strength }\end{array}$ & $\begin{array}{c}\text { Elonga- } \\
\text { tion in } \\
2 \text { in. }\end{array}$ & Remarks $^{s}$ & Pack number & Length & $\begin{array}{c}\text { Num- } \\
\text { ber of } \\
\text { speci- } \\
\text { mens } \\
\text { in pack }\end{array}$ & $\begin{array}{c}\text { Slope of } \\
\text { straight } \\
\text { line } 1\end{array}$ & $\begin{array}{c}\text { Yield } \\
\text { strength;2 } \\
\text { offset } \\
=0.2 \%\end{array}$ & $\begin{array}{l}\text { Cylinder } \\
\text { number }\end{array}$ & Length & $\begin{array}{c}\text { Out- } \\
\text { side di- } \\
\text { ameter, } \\
\text { nom- } \\
\text { inal }\end{array}$ & $\begin{array}{c}\text { Slope of } \\
\text { straight } \\
\text { line } 1\end{array}$ & $\begin{array}{l}\text { Yield } \\
\text { strength; } \\
\text { offset } \\
=0.2 \%\end{array}$ \\
\hline \multicolumn{17}{|c|}{0.010 IN. THICK, AS-ROLLED, LONGITUDINAL } \\
\hline $\begin{array}{r}\text { Percent } \\
0.0 \\
12.0 \\
19.6 \\
31.5 \\
40.0 \\
50.0 \\
52.5\end{array}$ & $\begin{array}{l}\text { 51-B1L } \\
50-\mathrm{B} 1 \mathrm{~L} \\
52-\mathrm{B} 1 \mathrm{~L} \\
53-\mathrm{B} 1 \mathrm{~L} \\
54-\mathrm{B} 2 \mathrm{~L} \\
59-\mathrm{B} 2 \mathrm{~L} \\
55-\mathrm{B} 1 \mathrm{~L}\end{array}$ & $\begin{array}{r}\text { Kips/in. }{ }^{2} \\
28,600 \\
26,500 \\
27,400 \\
26,700 \\
26,300 \\
25,800 \\
26,400\end{array}$ & $\begin{array}{r}\text { Kips/in. }{ }^{2} \\
36.7 \\
74.3 \\
102.6 \\
149.7 \\
174.5 \\
216.0 \\
246.4\end{array}$ & $\begin{array}{r}\text { Kips/in. } .^{2} \\
153.6 \\
170.0 \\
182.6 \\
204.0 \\
211.9 \\
231.7 \\
261.0\end{array}$ & $\begin{array}{r}\text { Percent } \\
60.5 \\
47.5 \\
35.5 \\
28.5 \\
25.0 \\
11.0 \\
3.0\end{array}$ & $\begin{array}{l}c \\
b \\
b \\
b \\
b \\
b \\
b\end{array}$ & \begin{tabular}{|l} 
None-1o \\
\\
\\
\end{tabular} & \begin{tabular}{|c|} 
Inches \\
\\
\end{tabular} & (1) & \begin{tabular}{|l} 
Kips/in. ${ }^{2}$ \\
\\
\end{tabular} & \begin{tabular}{|c|} 
Kips/in.2 \\
\\
\\
\end{tabular} & $\begin{array}{l}\text { 51-CX1L } \\
50-\mathrm{CX} 1 \mathrm{~L} \\
52-\mathrm{CX} 1 \mathrm{~L} \\
53-\mathrm{CX} 1 \mathrm{~L} \\
54-\mathrm{CX} 1 \mathrm{~L} \\
59-\mathrm{CX} 1 \mathrm{~L} \\
\text { 55-CX1L... }\end{array}$ & $\begin{array}{r}\text { Inches } \\
1.743 \\
1.742 \\
1.748 \\
1.749 \\
1.725 \\
1.746 \\
1.750\end{array}$ & $\begin{array}{r}\text { Inches } \\
0.40 \\
.40 \\
.40 \\
.40 \\
.40 \\
.40 \\
.40\end{array}$ & \begin{tabular}{|c|} 
Kips/in. \\
32,100 \\
30,400 \\
28,700 \\
26,900 \\
29,900 \\
26,000 \\
26,300
\end{tabular} & $\begin{array}{r}\text { Kips/in. } 2 \\
44.4 \\
59.7 \\
74.5 \\
100.9 \\
109.0 \\
142.2 \\
166.8\end{array}$ \\
\hline \multicolumn{17}{|c|}{0.010 IN. THICK, AS-ROLLED, TRANSVERSE } \\
\hline $\begin{array}{r}0.0 \\
12.0 \\
19.6 \\
31.5 \\
40.0 \\
50.0 \\
52.5\end{array}$ & $\begin{array}{l}\text { 51-B2T } \\
50-\mathrm{B} 2 \mathrm{~T} \\
52-\mathrm{B} 1 \mathrm{~T} \\
53-\mathrm{B} 1 \mathrm{~T} \\
54-\mathrm{B} 2 \mathrm{~T} \\
59-\mathrm{B} 1 \mathrm{~T} \\
55-\mathrm{B} 1 \mathrm{~T}\end{array}$ & $\begin{array}{l}28,400 \\
27,400 \\
27,800 \\
28,200 \\
28,900 \\
30,400 \\
30,700\end{array}$ & $\begin{array}{r}36.4 \\
72.5 \\
100.2 \\
136.7 \\
154.4 \\
197.0 \\
218.5\end{array}$ & $\begin{array}{l}154.4 \\
167.2 \\
180.1 \\
201.5 \\
217.7 \\
244.8 \\
285.1\end{array}$ & $\begin{array}{r}60.0 \\
47.0 \\
37.5 \\
22.5 \\
21.5 \\
11.0 \\
4.5\end{array}$ & $\begin{array}{l}\mathrm{b} \\
\mathrm{b} \\
\mathrm{b} \\
\mathrm{b} \\
\mathrm{c} \\
\mathrm{d}(9.0) \\
\mathrm{b}\end{array}$ & 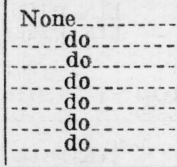 & $\mid$ & $\mid$ & \begin{tabular}{|c|}
$\ldots$ \\
\hdashline
\end{tabular} & \begin{tabular}{|c|}
-1 \\
-1
\end{tabular} & 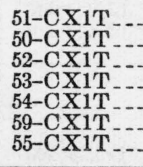 & $\begin{array}{l}1.732 \\
1.751 \\
1.743 \\
1.748 \\
1.728 \\
1.746 \\
1.744\end{array}$ & $\begin{array}{r}0.40 \\
.40 \\
.40 \\
.40 \\
.40 \\
.40 \\
.40\end{array}$ & $\begin{array}{l}31,600 \\
28,800 \\
31,500 \\
28,300 \\
29,900 \\
30,200 \\
31,100\end{array}$ & $\begin{array}{r}41.5 \\
79.4 \\
112.1 \\
163.8 \\
180.7 \\
231.6 \\
248.2\end{array}$ \\
\hline \multicolumn{17}{|c|}{0.020 IN. THICK, AS-ROLLED, LONGITUDINAL } \\
\hline $\begin{array}{r}0.0 \\
5.3 \\
18.3 \\
29.8 \\
37.5 \\
50.0\end{array}$ & $\begin{array}{l}\text { 68-B1L } \\
69-\mathrm{B} 2 \mathrm{~L} \\
\text { 45-B1L } \\
47-\mathrm{B} 2 \mathrm{~L} \\
66-\mathrm{B} 2 \mathrm{~L} \\
49-\mathrm{B} 1 \mathrm{~L}\end{array}$ & $\begin{array}{l}28,400 \\
27,800 \\
26,600 \\
27,000 \\
26,300 \\
26,300\end{array}$ & \begin{tabular}{r|}
36.2 \\
80.4 \\
115.5 \\
138.7 \\
152.6 \\
201.6
\end{tabular} & $\begin{array}{l}152.1 \\
169.9 \\
186.3 \\
198.9 \\
205.1 \\
236.8\end{array}$ & $\begin{array}{l}63.0 \\
49.0 \\
32.5 \\
28.0 \\
25.0 \\
27.5\end{array}$ & $\begin{array}{l}b \\
b \\
b \\
b \\
b \\
c\end{array}$ & $\begin{array}{l}\text { 57-C2L } \\
\text { 58-C1L } \\
44-\mathrm{C} 2 \mathrm{~L} \\
46-\mathrm{C} 2 \mathrm{~L} \\
66-\mathrm{CP} 4 \mathrm{~L} \\
49-\mathrm{CN} 1 \mathrm{~L}\end{array}$ & $\begin{array}{l}\text { 3. } 501 \\
3.500 \\
3.497 \\
3.498 \\
2.500 \\
2.502\end{array}$ & \begin{tabular}{|r|}
31 \\
31 \\
31 \\
31 \\
7 \\
71
\end{tabular} & $\begin{array}{l}29,500 \\
28,500 \\
27,800 \\
27,400 \\
27,000 \\
26,400\end{array}$ & $\begin{array}{r}36.7 \\
47.4 \\
82.0 \\
94.3 \\
104.7 \\
135.1\end{array}$ & $\begin{array}{l}\text { 57-CX1L } \\
\text { 58-CX1L } \\
\text { 45-CX1L } \\
\text { 47-CX1L } \\
\text { 56-CX1L } \\
\text { 49-CX1L }\end{array}$ & $\begin{array}{l}1.994 \\
1.993 \\
1.992 \\
1.996 \\
1.994 \\
1.998\end{array}$ & $\begin{array}{r}0.80 \\
.80 \\
.80 \\
.80 \\
.80 \\
.80\end{array}$ & $\begin{array}{l}30,700 \\
26,400 \\
26,300 \\
26,700 \\
25,700 \\
24,800\end{array}$ & $\begin{array}{r}43.8 \\
66.8 \\
87.8 \\
101.7 \\
120.4 \\
147.8\end{array}$ \\
\hline
\end{tabular}

\begin{tabular}{|c|c|c|c|c|c|c|c|c|c|c|c|c|c|c|c|c|}
\hline 0.0 & 68-B1T & 28,000 & 35.8 & 153.1 & 62.0 & b & $57-\mathrm{C} 1 \mathrm{~T}$. & 3. 502 & 31 & 29,800 & 37.8 & 57-CX1T. & 1.995 & 0.80 & 31,800 & 43.1 \\
\hline 5.3 & $69-\mathrm{B} 2 \mathrm{~T}$ & 27,700 & 82.9 & 174.1 & 44.5 & b & $58-\mathrm{C} 1 \mathrm{~T}$. & 3. 500 & 31 & 28,800 & 88.1 & $58-\mathrm{C} \times 1 \mathrm{~T}$ & 1.995 & .80 & 28,600 & 86.6 \\
\hline 18.3 & 45-B2T & 28,100 & 115.8 & 186. 3 & 37.0 & b & 44-C1T & 3. 502 & 31 & 28,500 & 132.0 & $45-\mathrm{CX} 1 \mathrm{~T}$ & 1. 996 & .80 & 28,800 & 127.7 \\
\hline 29.8 & 47-B2T & 28,800 & 136.6 & 203.5 & 29.0 & & 47-СР6T 7 & 2. 500 & 7 & 28,900 & 158.1 & 47-CX1T. & $\begin{array}{l}\text { 1. } 996 \\
\text { 1. }\end{array}$ & .80 & 29.300 & 155.3 \\
\hline 37.5 & $66-\mathrm{B} 2 \mathrm{~T}$ & 28,600 & 154.7 & 208.2 & 27.0 & $\mathrm{~d}(24.0)$ & 66-CP3T ${ }^{7}$ & 2. 500 & 7 & 29,500 & 173.8 & $56-\mathrm{CX} 2 \mathrm{~T}$ & 1. 999 & .80 & 29,000 & 173. 1 \\
\hline 50.0 & $49-\mathrm{B} 1 \mathrm{~T}$ & 30,400 & 198.3 & 243.2 & 6.0 & b & $48-\mathrm{CS} 2 \mathrm{~T}$ & 1. 801 & 31 & 31,800 & 228.8 & $49-\mathrm{CX} 1 \mathrm{~T}$ & 1. 994 & .80 & 29,600 & 212.5 \\
\hline
\end{tabular}


0.035 IN. THICK, AS-ROLLED, LONGITUDINAL

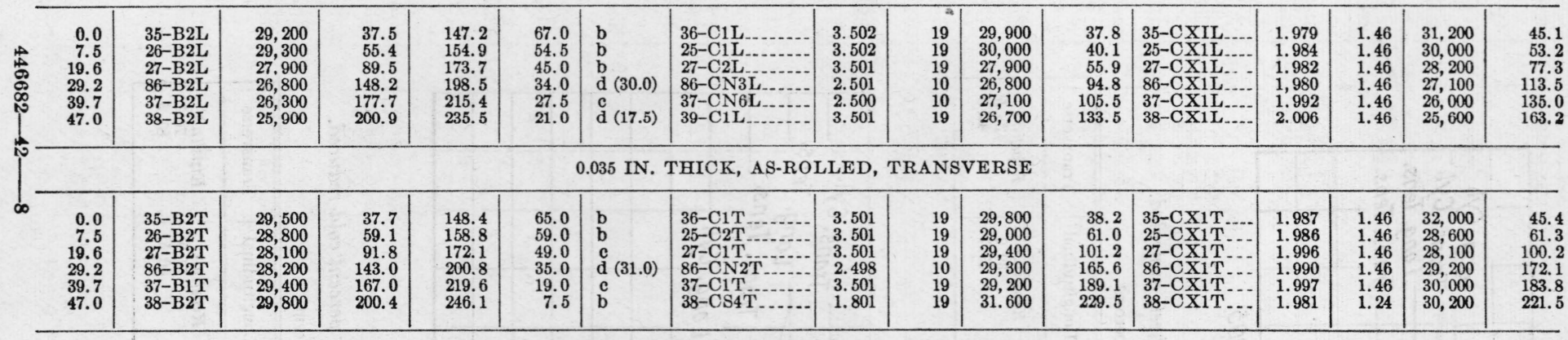

$0.060 \mathrm{IN}$. THICK, AS-ROLLED, LONGITUDINAL

\begin{tabular}{|c|c|c|c|c|c|c|c|c|c|c|c|c|c|c|c|c|}
\hline $\begin{array}{r}0.0 \\
6.7 \\
18.1 \\
30.5 \\
36.9 \\
47.7\end{array}$ & $\begin{array}{c}\text { 41-B1L } \\
7-\mathrm{B} 2 \mathrm{~L} \\
\text { 8-B2L } \\
\text { 11-B2L } \\
29-\mathrm{B} 1 \mathrm{~L} \\
13-\mathrm{B} 1 \mathrm{~L}\end{array}$ & $\begin{array}{l}28,600 \\
26,900 \\
27,700 \\
26,600 \\
26,300 \\
26,300\end{array}$ & $\begin{array}{r}34.1 \\
81.5 \\
103.7 \\
148.6 \\
173.7 \\
194.3\end{array}$ & $\begin{array}{r}146.9 \\
\text { '168. } \\
178.7 \\
202.2 \\
209.5 \\
225.0\end{array}$ & $\begin{array}{r}72.5 \\
+52.5 \\
37.5 \\
31.0 \\
27.0 \\
16.0\end{array}$ & $\begin{array}{l}\mathrm{b} \\
\mathrm{b} \\
\mathrm{b} \\
\mathrm{c} \\
\mathrm{c} \\
\mathrm{c}\end{array}$ & 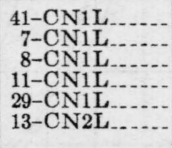 & $\begin{array}{l}2.485 \\
2.488 \\
2.463 \\
2.479 \\
2.486 \\
2.474\end{array}$ & $\begin{array}{l}5 \\
5 \\
5 \\
5 \\
5 \\
5\end{array}$ & $\begin{array}{l}31,400 \\
27,600 \\
27,700 \\
27,200 \\
26,900 \\
26,600\end{array}$ & $\begin{array}{r}35.3 \\
57.8 \\
69.4 \\
97.4 \\
104.3 \\
125.1\end{array}$ & \begin{tabular}{|l} 
None \\
do do \\
\\
\\
\end{tabular} & 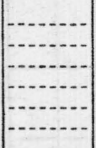 & \begin{tabular}{|c|}
-1 \\
\hdashline
\end{tabular} & & 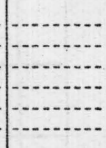 \\
\hline
\end{tabular}

0.060 IN. THICK, AS-ROLLED, TRANSVERSE

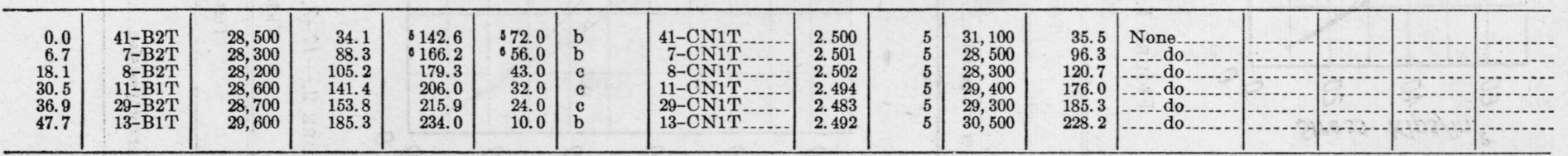

1 Obtained by least-square fit to lower portion of stress-strain graph.

$541-\mathrm{B} 2 \mathrm{~T}$ broke in grip; duplicate specimen (41-B1T) tested for tensile strength and ${ }^{3}$ Letters describe location of fracture in accordance with section 24 and figure 19, page valos $A$ derminged

Loaded at $0.02 \mathrm{in}$. $/ \mathrm{min}$.

elongation. strength and $\mathrm{e}$.

${ }^{7}$ One-half-inch-wide pack, tested in subpress, lateral support 39 pins on each side, 3̧o-in. spacing. 


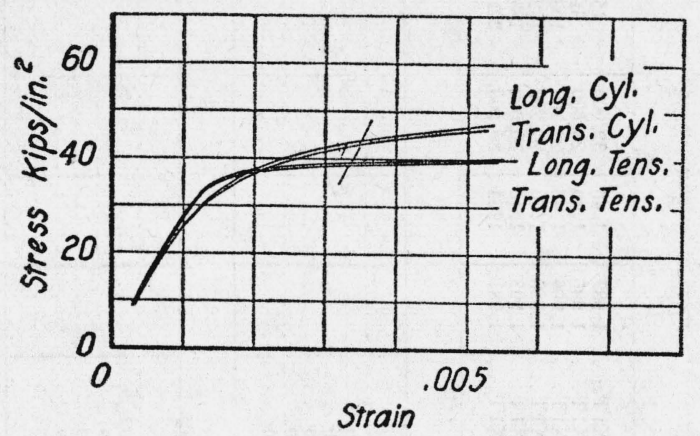

Figure 1.-17-7, 0.01-inch sheet, annealed.

Yield strength, offset $=0.2$ percent

\begin{tabular}{|c|c|c|}
\hline Test & Longitudinal & Transverse \\
\hline $\begin{array}{l}\text { Tensile } \\
\text { Cylinder compressive }\end{array}$ & $\begin{array}{r}\text { Kips/in. } \\
39.0 \\
43.7\end{array}$ & $\begin{array}{r}\text { Kips/in.' } \\
38.5 \\
42.6\end{array}$ \\
\hline
\end{tabular}

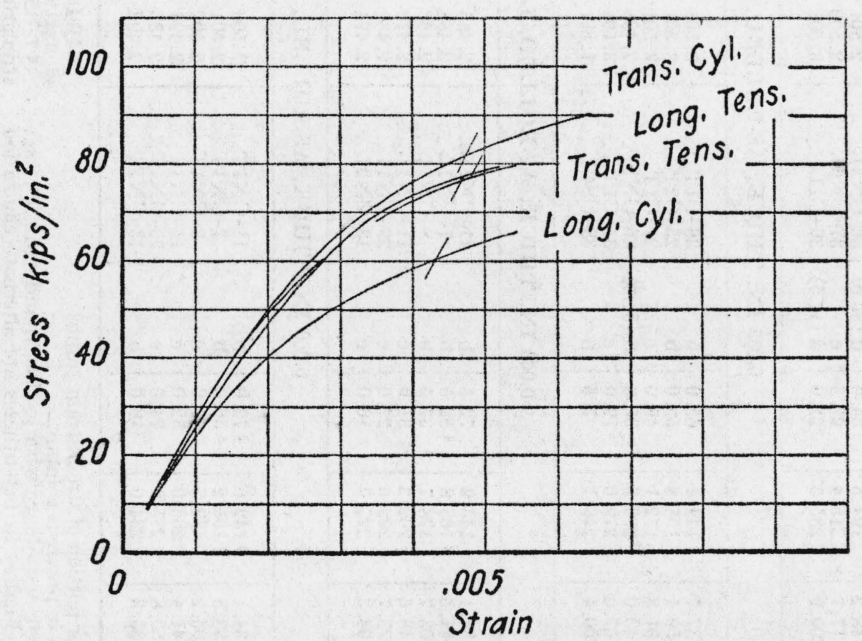

Figure 2.-17-7, 0.01-inch sheet, 8.2 percent cold-reduced. Yield strength, offset $=0.2$ percent

\begin{tabular}{|c|r|r|}
\hline Test & Longitudinal & Transverse \\
\cline { 2 - 3 } & & Kips/in.2. \\
$\mathbf{7 7 . 9}$ & $\begin{array}{r}\text { Kips/in..2 } \\
76.7 \\
81.5\end{array}$ \\
\hline
\end{tabular}




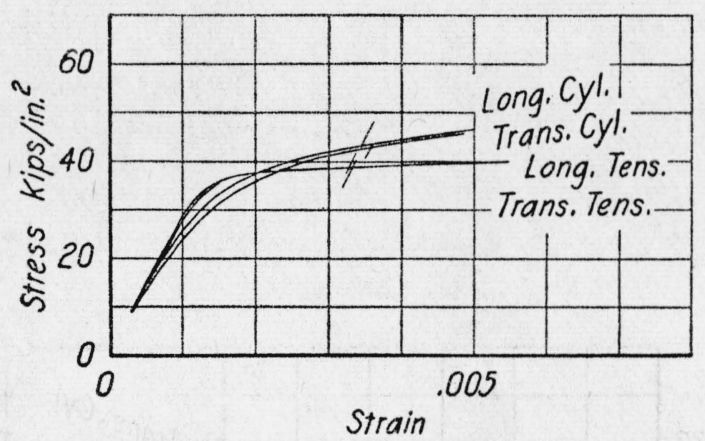

FIGURE 3.-17-7, 0.01-inch sheet, annealed, stress-relieved. Yield strength, offset $=0.2$ percent

\begin{tabular}{|r|r|r|}
\hline Test & Longitudinal & Transverse \\
\cline { 2 - 3 } & & Kips/in.2.2.2. \\
39.2 & $\begin{array}{r}\text { Kips/in.2 } \\
39.0 \\
42.9\end{array}$ \\
\hline
\end{tabular}

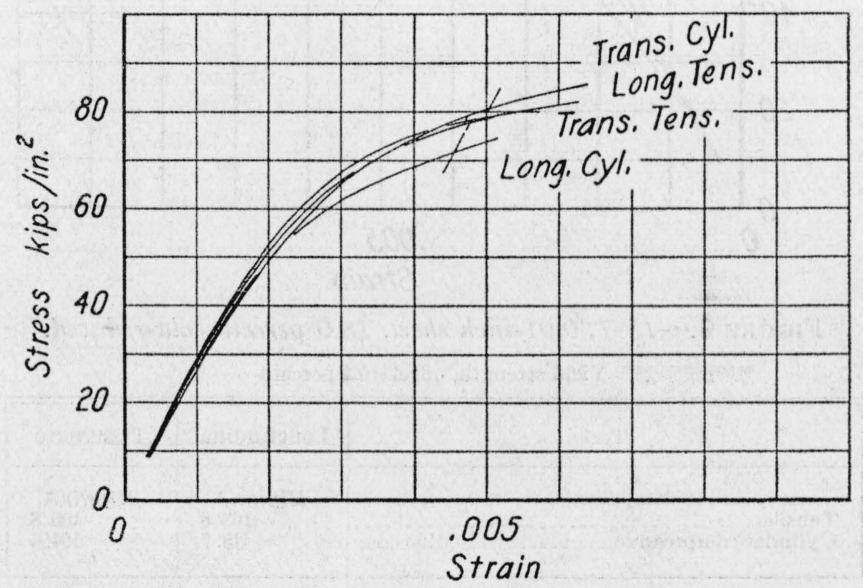

Figure 4.-17-7, 0.01-inch sheet, 8.2 percent cold-reduced, stress-relieved. Yield strength, offset $=0.2$ percent

\begin{tabular}{|c|c|c|}
\hline Test & Longitudinal & Transverse \\
\hline 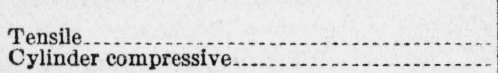 & $\begin{array}{r}\text { Kips/in.8 } \\
78.2 \\
71.6\end{array}$ & $\begin{array}{r}\text { Kips/in.8 } \\
77.7 \\
80.3\end{array}$ \\
\hline
\end{tabular}




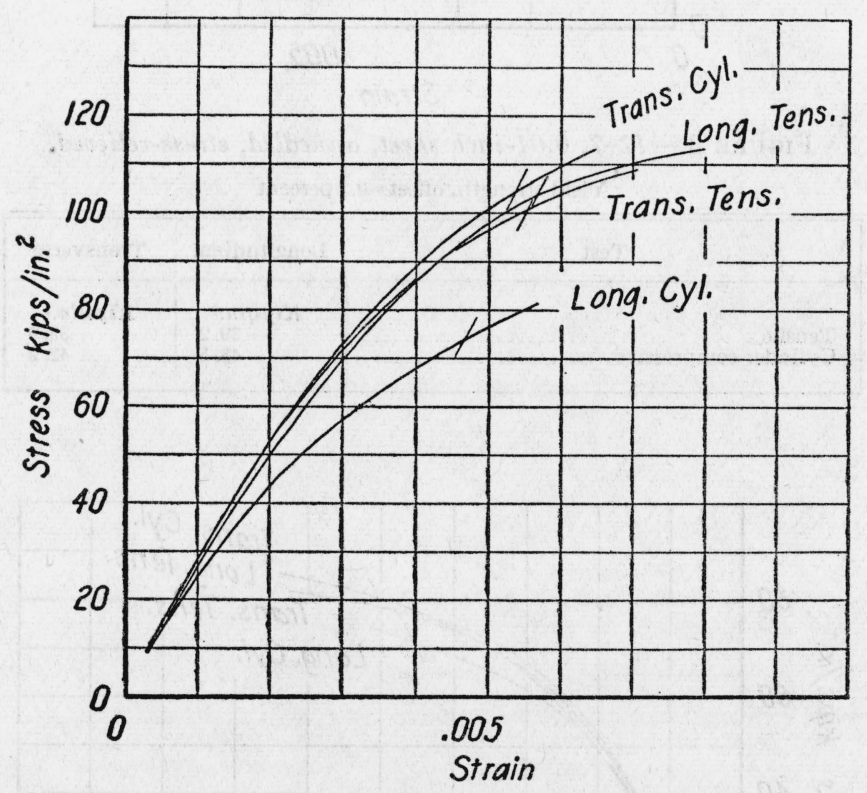

FiguRe 5.-17-7, 0.01-inch sheet, 18.0 percent cold-reduced.

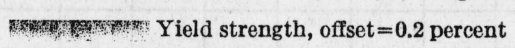

\begin{tabular}{|c|r|r|}
\hline Test & Longitudinal & Transverse \\
\cline { 1 - 2 } & $\begin{array}{r}\text { Kips/in.8 } \\
103.8 \\
73.7\end{array}$ & $\begin{array}{r}\text { Kips/in.2. } \\
100.8 \\
104.4\end{array}$ \\
\hline
\end{tabular}




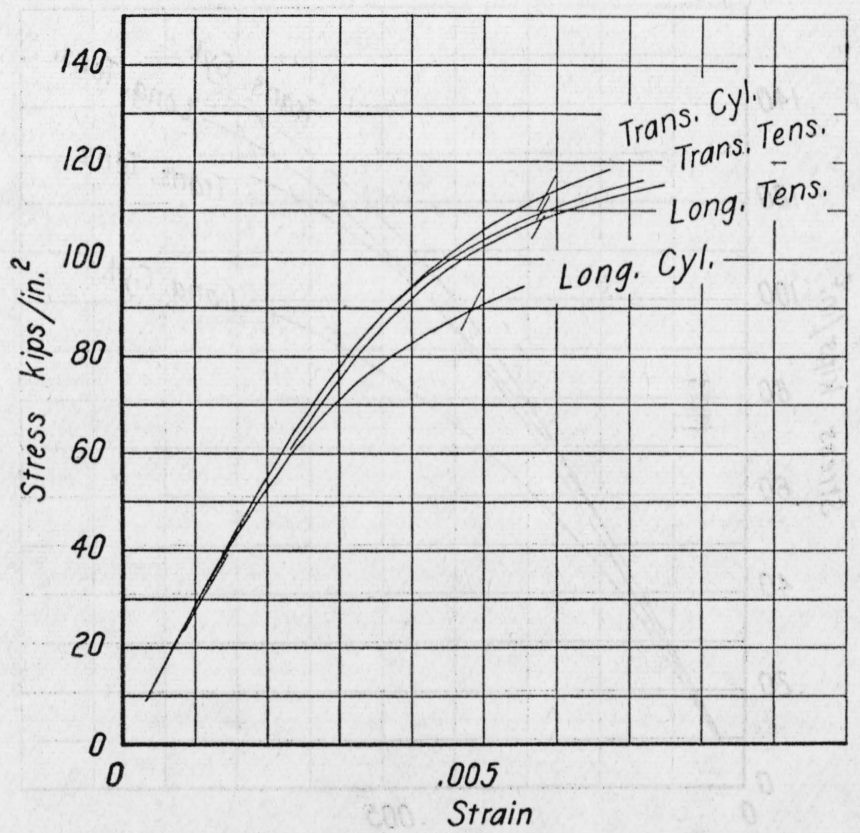

FIGURE 6.-17-7, 0.01-inch sheet, 18.0 percent cold-reduced, stress-relieved. Yield strength, offset $=0.2$ percent

\begin{tabular}{|c|c|c|}
\hline Test & Longitudinal & Transverse \\
\hline $\begin{array}{l}\text { Tensile } \\
\text { Cylinder compressive }\end{array}$ & $\begin{array}{r}\text { Kips/in.2 } \\
108.4 \\
89.7\end{array}$ & $\begin{array}{r}\text { Kips/in.2. } \\
109.5 \\
112.7\end{array}$ \\
\hline
\end{tabular}


516 Journal of Research of the National Bureau of Standards

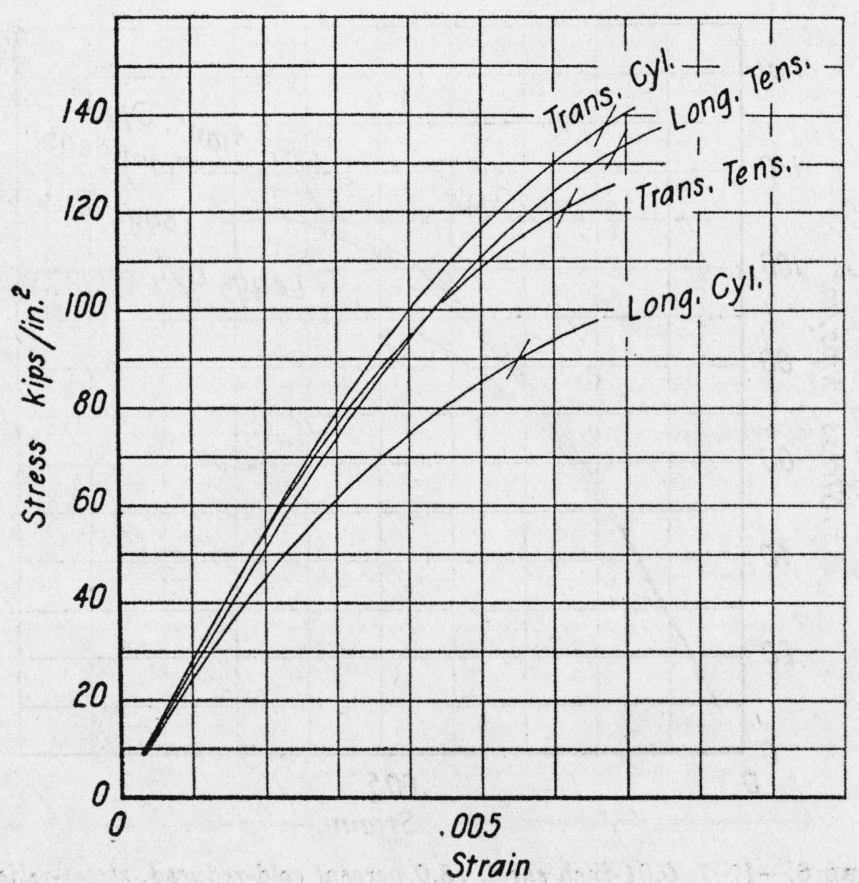

FrguRE 7.-17-7, 0.01-inch sheet, 28.0 percent cold-reduced. Yield strength, offset $=0.2$ percent

\begin{tabular}{|r|r|r|}
\hline Test & Longitudinal & Transverse \\
\hline & Kips/in.2.2. \\
133.1 & $\begin{array}{r}\text { Kips/in.2. } \\
121.0 \\
137.8\end{array}$ \\
\hline
\end{tabular}




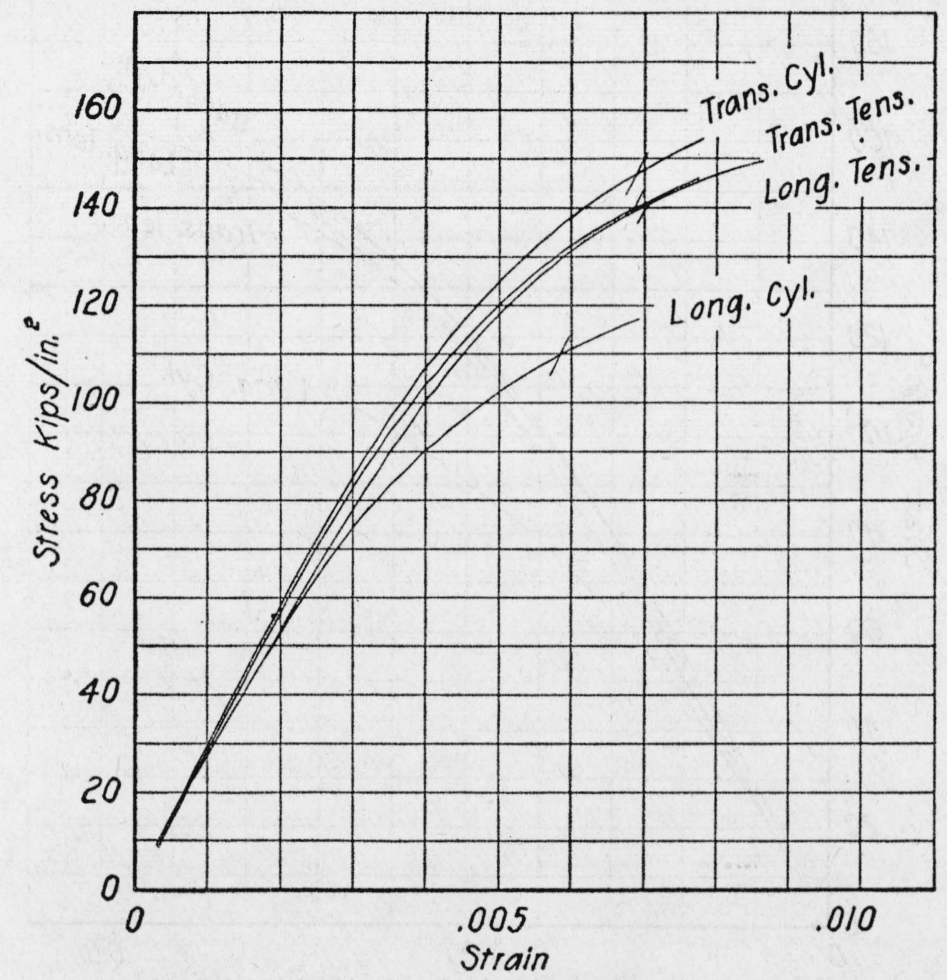

Figure 8.-17-7, 0.01-inch sheet, 28.0 percent cold-reduced, stress-relieved.

Yield strength, offset $=0.2$ percent

\begin{tabular}{|r|r|r|}
\hline Test & Longitudinal & Transverse \\
\cline { 2 - 3 } & Kips/in.8 \\
Tensile & 141.5 & $\begin{array}{r}\text { Kips/in.2. } \\
139.8 \\
146.6\end{array}$ \\
\hline
\end{tabular}




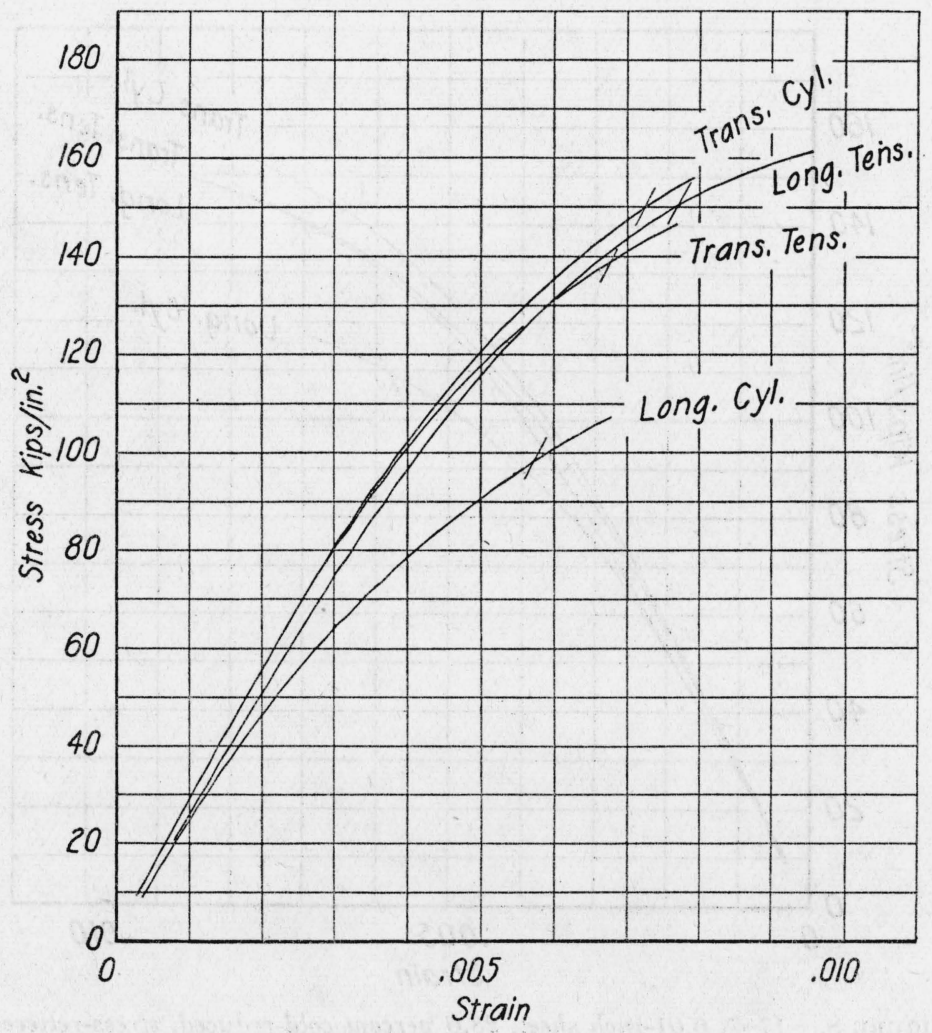

Frgure 9. $-17-7,0.01$-inch sheet, 37.0 percent cold-reduced. Yield strength, offset $=0.2$ percent

\begin{tabular}{|c|r|r|}
\hline Test & Longitudinal & Transverse \\
\hline Tensile & Kips/in.2. & Kips/in..$^{2}$ \\
Cylinder compressive. & 138.8 \\
& 98.3 & 150.0 \\
\hline
\end{tabular}




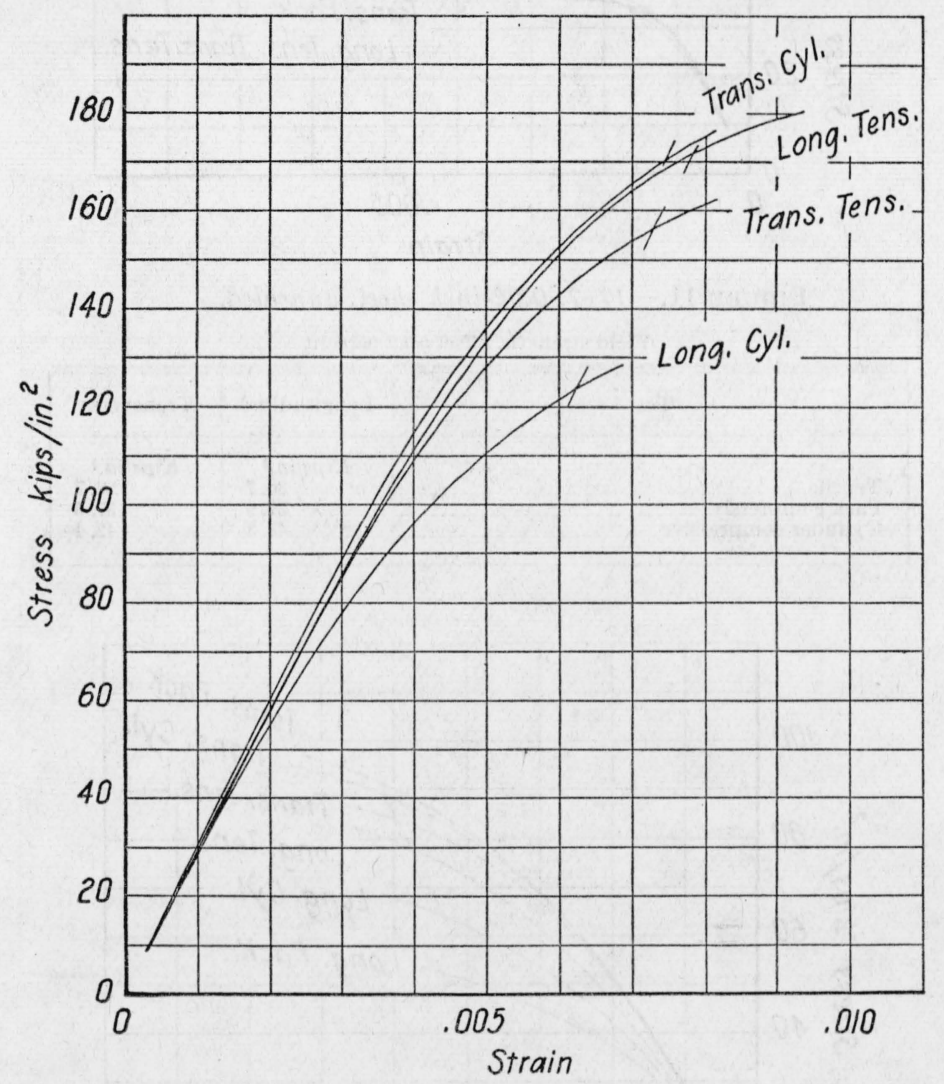

FigURE 10.-17-7, 0.01-inch sheet, 37.0 percent cold-reduced, stress-relieved.

Yield strength, offset $=0.2$ percent

\begin{tabular}{|c|c|c|}
\hline Test & Longitudinal & Transverse \\
\hline $\begin{array}{l}\text { Tensile } \\
\text { Cylinder compressive }\end{array}$ & $\begin{array}{r}\text { Kips/in. } .^{2} \\
171.7 \\
123.4\end{array}$ & $\begin{array}{r}\text { Kips/in. }{ }^{2} \\
156.5 \\
170.4\end{array}$ \\
\hline
\end{tabular}




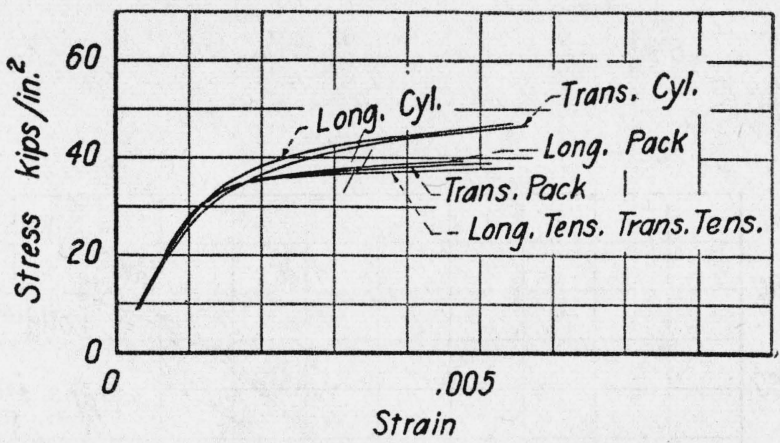

Figure 11.-17-7, 0.02-inch sheet, annealed.

Yield strength, offset $=0.2$ percent

\begin{tabular}{|c|c|c|}
\hline Test & Longitudinal & Transverse \\
\hline $\begin{array}{l}\text { Tensile } \\
\text { Pack compressive } \\
\text { Cylinder compressive }\end{array}$ & $\begin{array}{r}\text { Kips/in. } .^{2} \\
36.7 \\
37.9 \\
43.3\end{array}$ & $\begin{array}{r}\text { Kips/in. }{ }^{2} \\
36.7 \\
37.5 \\
42.4\end{array}$ \\
\hline
\end{tabular}

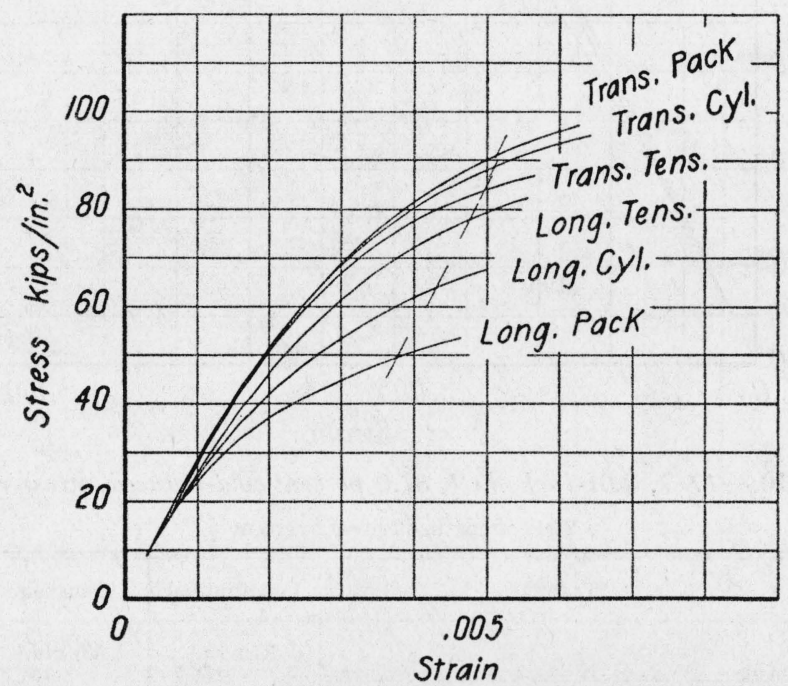

FIGURE 12.-17- $7,0.02-$ inch sheet, 8.2 percent cold-reduced.

Yield strength, offset $=0.2$ percent

\begin{tabular}{|c|c|c|}
\hline Test & Longitudinal & Transverse \\
\hline $\begin{array}{l}\text { Tensile } \\
\text { Pack compressive } \\
\text { Cylinder compressive.................... }\end{array}$ & $\begin{array}{r}\text { Kips/in. } \\
78.3 \\
49.4 \\
64.0\end{array}$ & $\begin{array}{r}\text { Kips/in.2 } \\
84.0 \\
91.1 \\
88.9\end{array}$ \\
\hline
\end{tabular}




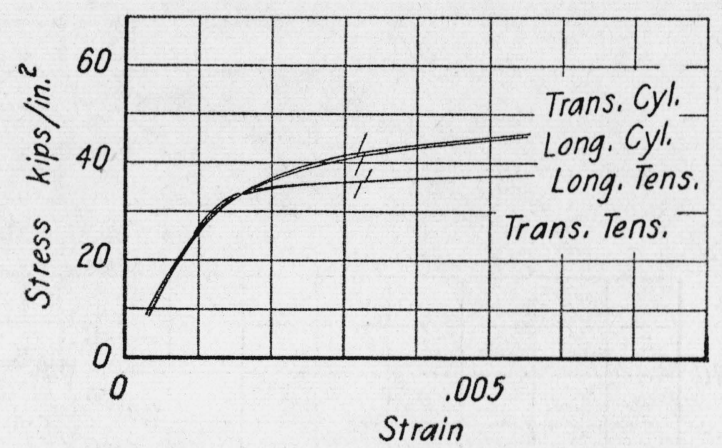

FIGURE 13.-17-7, 0.02-inch sheet, annealed, stress-relieved.

Yield strength, offset $=0.2$ percent

\begin{tabular}{|c|c|c|}
\hline Test & Longitudinal & Transverse \\
\hline $\begin{array}{l}\text { Tensile } \\
\text { Cylinder compressive }\end{array}$ & $\begin{array}{r}\text { Kips/in. } .^{2} \\
36.2 \\
41.7\end{array}$ & $\begin{array}{r}\text { Kips/in } .^{2} \\
36.0 \\
42.0\end{array}$ \\
\hline
\end{tabular}

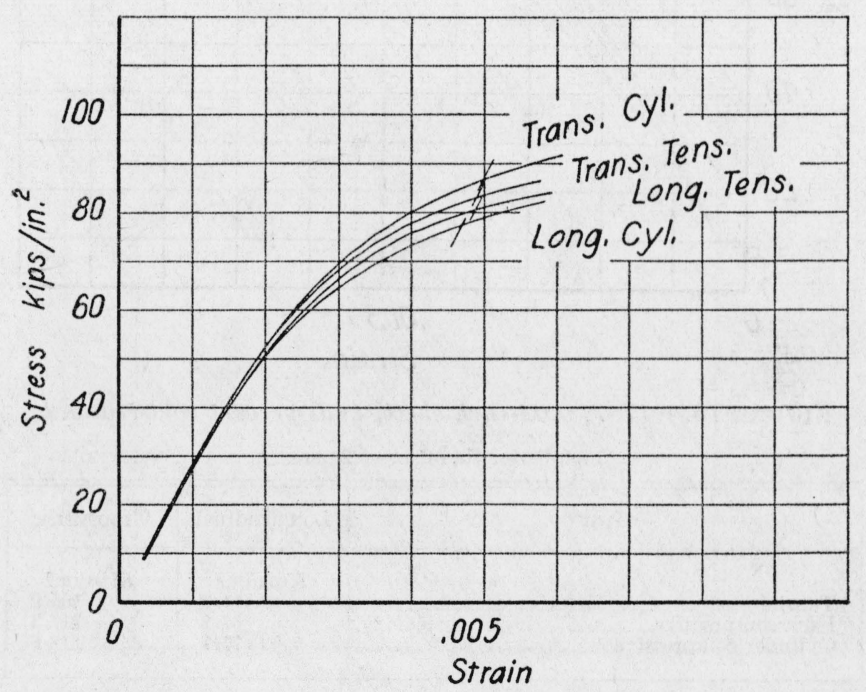

FiguRe 14.-17-7, 0.02-inch sheet, 8.2 percent cold-reduced, stress-relieved.

\begin{tabular}{|c|c|c|}
\hline Test & Longitudinal & Transverse \\
\hline $\begin{array}{l}\text { Tensile } \\
\text { Cylinder compressive }\end{array}$ & $\begin{array}{r}\text { Kips/in. } .^{2} \\
80.4 \\
77.5\end{array}$ & $\begin{array}{r}\text { Kips/in.' } \\
83.1 \\
86.8\end{array}$ \\
\hline
\end{tabular}




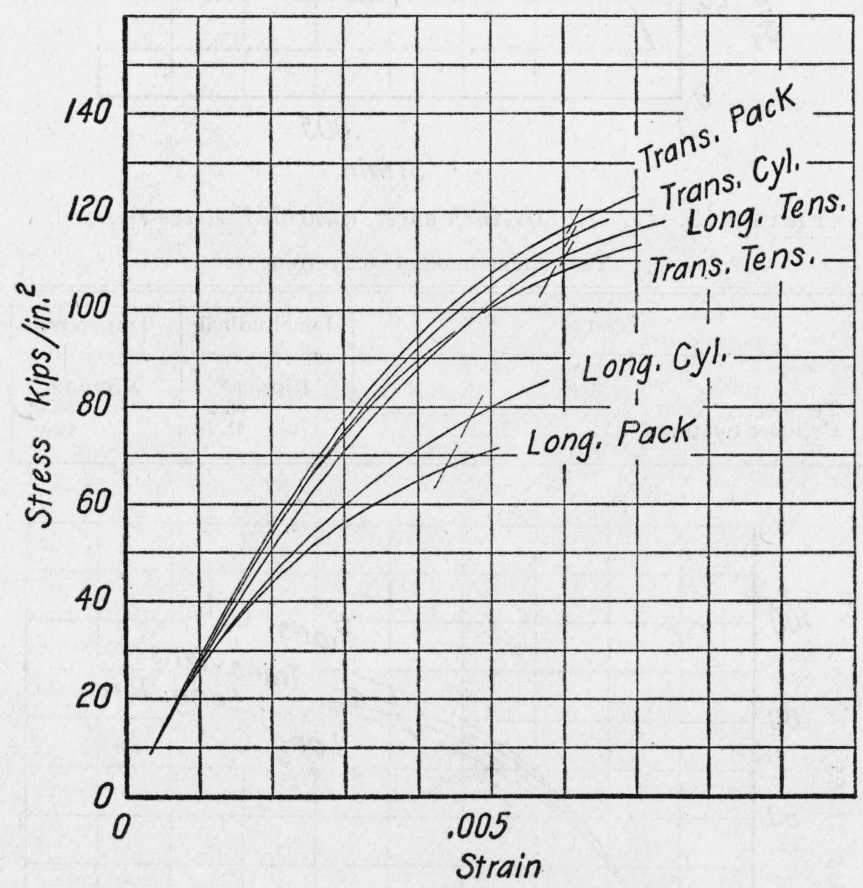

FigURE 15.-17-7, 0.02-inch sheet, 18.0 percent cold-reduced.

Yield"strength, offset $=0.2$ percent

\begin{tabular}{|c|c|c|}
\hline Test & Longitudinal & Transverse \\
\hline $\begin{array}{l}\text { Tensile } \\
\text { Pack compressive } \\
\text { Cylinder compressive }\end{array}$ & $\begin{array}{r}\text { Kips/in.2 } \\
111.0 \\
67.2 \\
77.7\end{array}$ & $\begin{array}{r}\text { Kips/in.2 } \\
106.9 \\
117.3 \\
114.8\end{array}$ \\
\hline
\end{tabular}




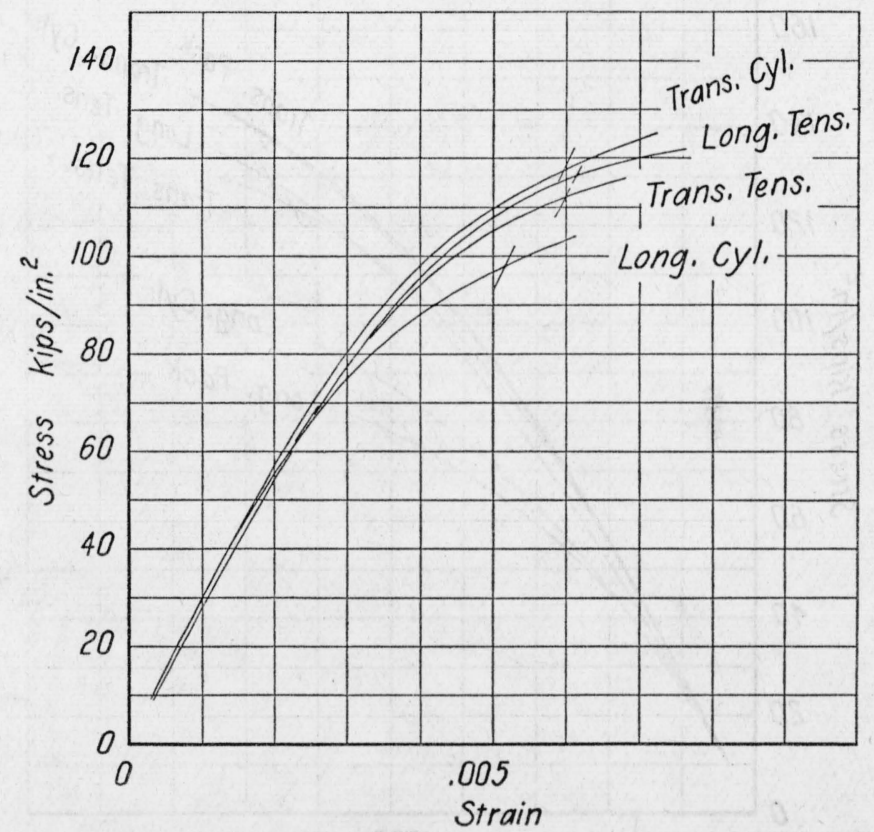

FIGURE 16.-17-7, 0.02-inch sheet, 18.0 percent cold-reduced, stress-relieved. Yield strength, offset $=0.2$ percent

\begin{tabular}{|c|c|c|}
\hline Test & Longitudinal & Transverse \\
\hline $\begin{array}{l}\text { Tensile } \\
\text { Cylinder compressive. }\end{array}$ & $\begin{array}{r}\text { Kips/in. } .^{2} \\
116.4 \\
98.0\end{array}$ & $\begin{array}{r}\text { Kips/in.2 } \\
112.1 \\
117.8\end{array}$ \\
\hline
\end{tabular}


524 Journal of Research of the National Bureau of Standards

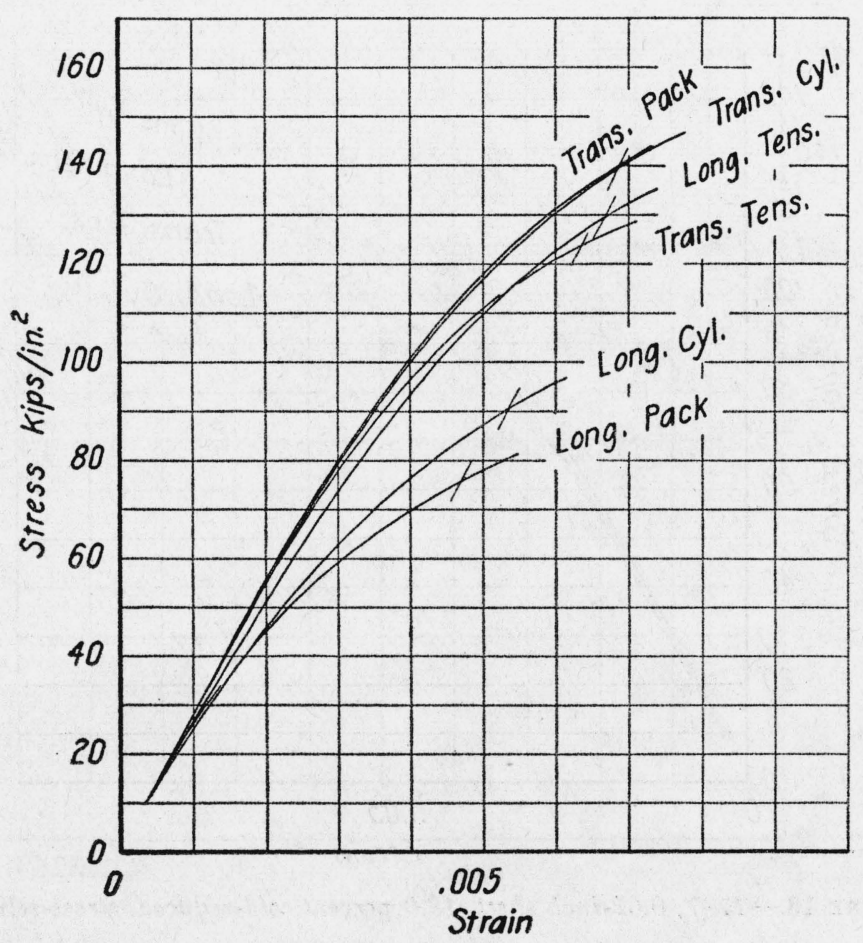

FiguRe 17.-17-7, 0.02-inch sheet, 30.5 percent cold-reduced.

Yield strength, offset $=0.2$ percent

\begin{tabular}{|c|c|c|}
\hline Test & Longitudinal & Transverse \\
\hline 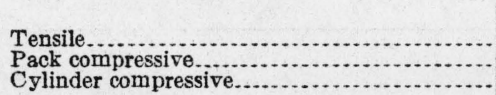 & $\begin{array}{r}\text { Kips/in.2 } \\
129.8 \\
75.4 \\
90.6\end{array}$ & $\begin{array}{r}\text { Kips/in.2 } \\
123.9 \\
140.0 \\
139.2\end{array}$ \\
\hline
\end{tabular}




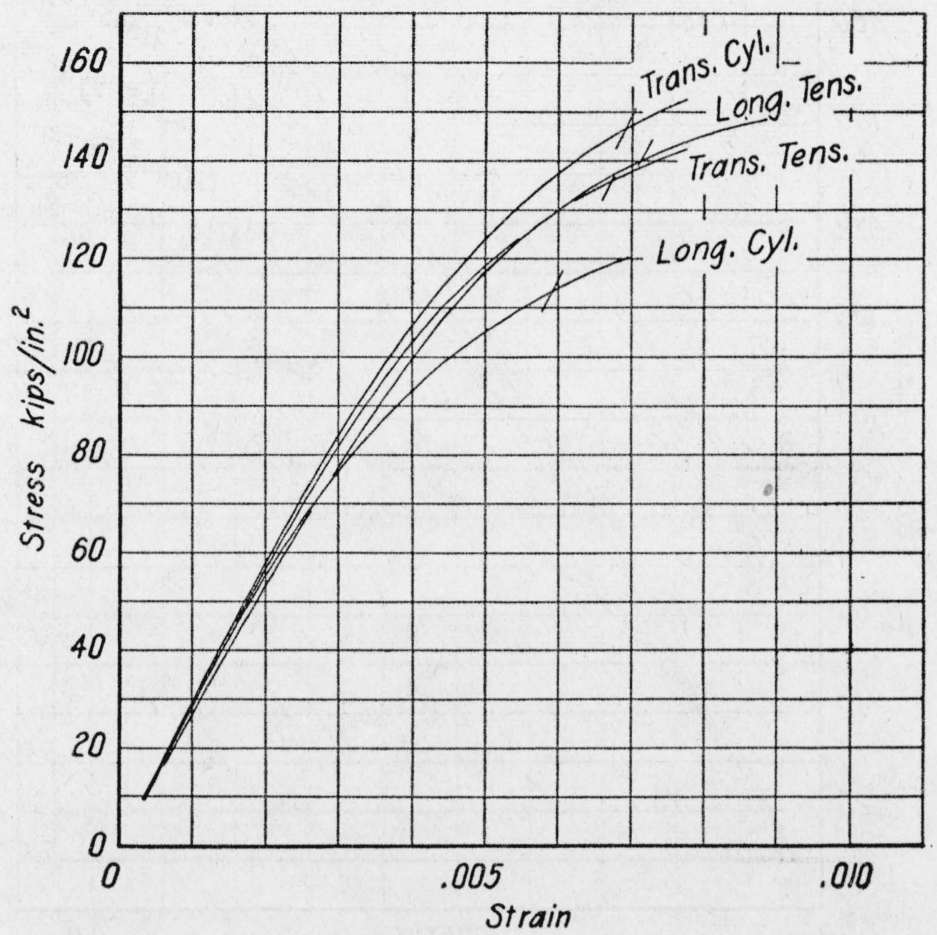

FIGURE 18.-17-7, 0.0\%-inch sheet, 30.5 percent cold-reduced, stress-relieved. Yield strength, offset $=0.2$ percent

\begin{tabular}{|c|c|c|}
\hline Test & Longitudinal & Transverse \\
\hline $\begin{array}{l}\text { Tensile } \\
\text { Cylinder compressive }\end{array}$ & $\begin{array}{r}\text { Kips/in. } .^{2} \\
140.0 \\
113.0\end{array}$ & $\begin{array}{r}\text { Kips/in. } .^{2} \\
136.0 \\
146.7\end{array}$ \\
\hline
\end{tabular}




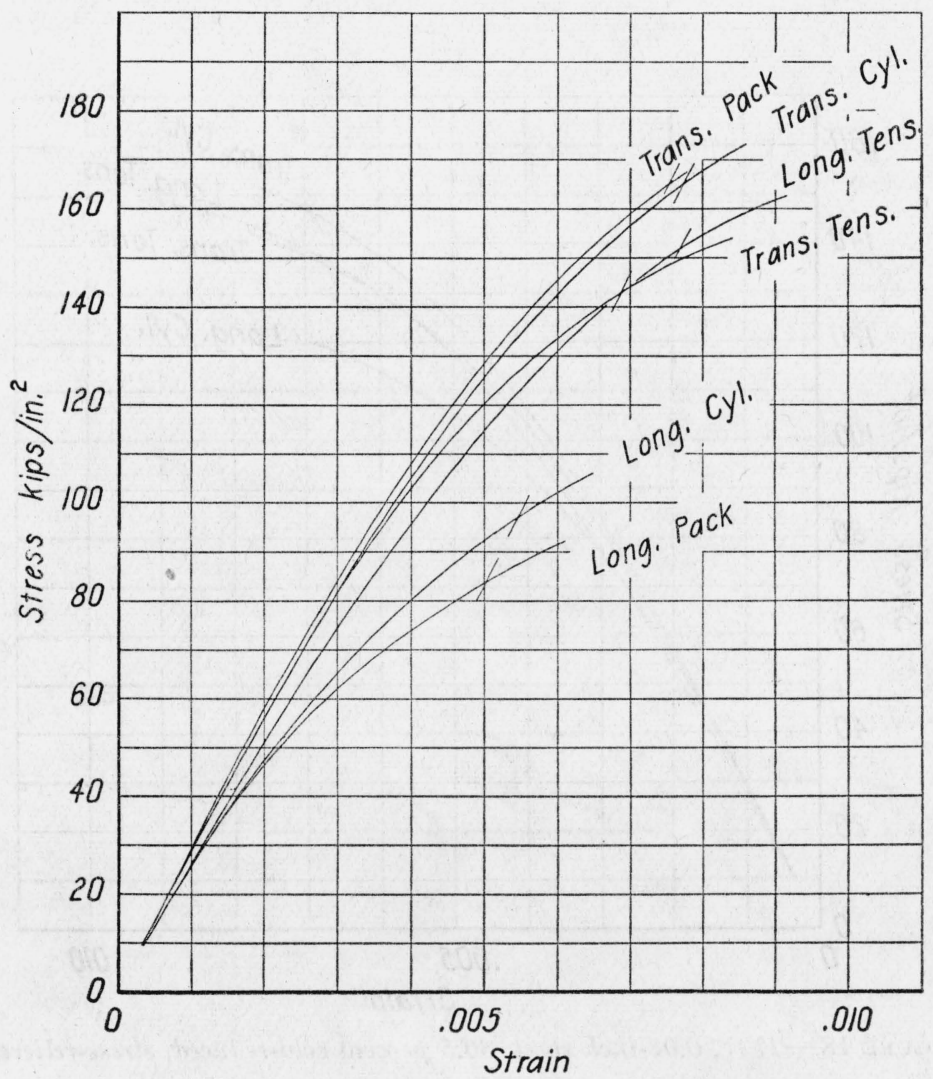

Figure 19.-17-7, 0.02-inch sheet, 37.5 percent cold-reduced. Yield strength, offset $=0.2$ percent

\begin{tabular}{|c|c|c|}
\hline Test & Longitudinal & Transverse \\
\hline $\begin{array}{l}\text { Tensile } \\
\text { Pack compressive } \\
\text { Cylinder compressive }\end{array}$ & $\begin{array}{r}\text { Kips/in. } .^{2} \\
151.9 \\
83.8 \\
96.7\end{array}$ & $\begin{array}{r}\text { Kips/in.2 } \\
143.0 \\
165.0 \\
165.0\end{array}$ \\
\hline
\end{tabular}




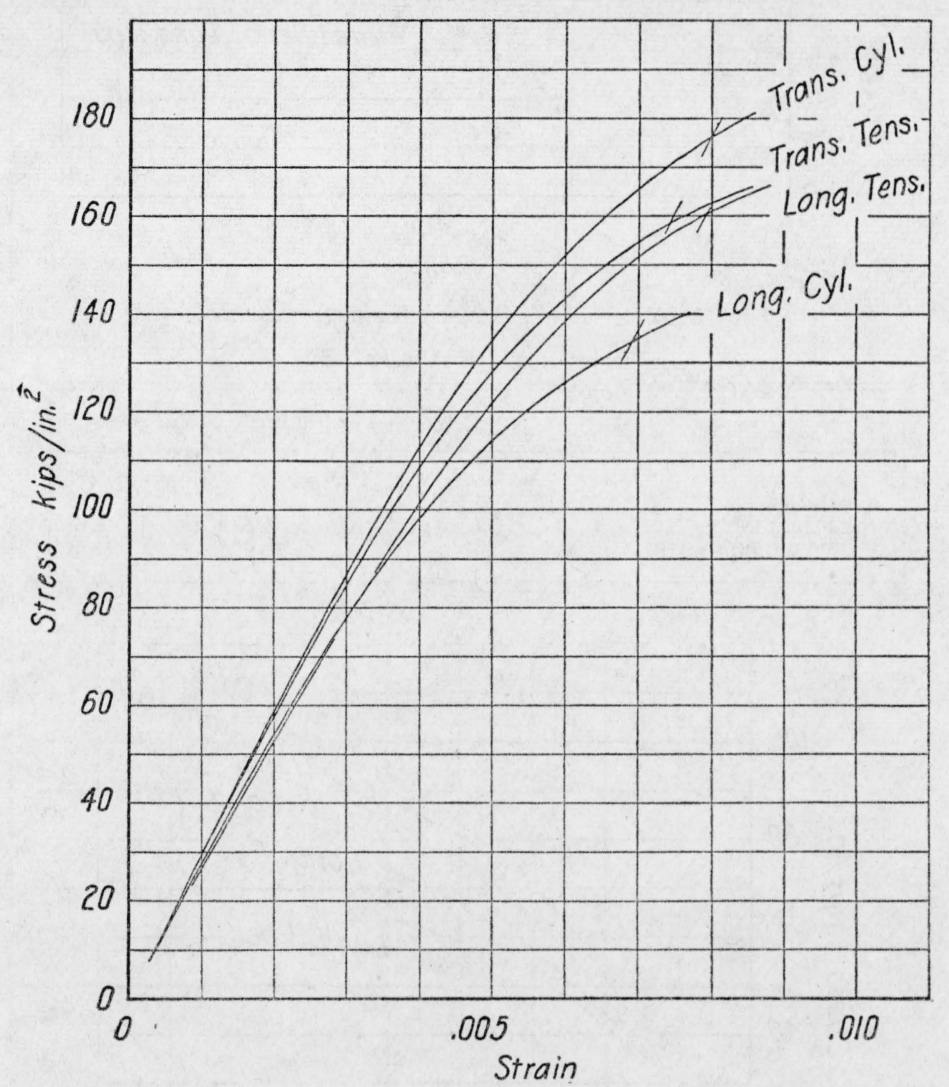

FIGURE 20.-17-7, 0.02-inch sheet, 37.5 percent cold-reduced, stress-relieved.

Yield strength, offset $=0.2$ percent

\begin{tabular}{|c|c|c|}
\hline Test & Longitudinal & Transverse \\
\hline $\begin{array}{l}\text { Tensile } \\
\text { Cylinder compressive }\end{array}$ & $\begin{array}{r}\text { Kips/in. } .^{2} \\
160.4 \\
134.5\end{array}$ & $\begin{array}{r}\text { Kips/in. } .^{2} \\
158.6 \\
176.4\end{array}$ \\
\hline
\end{tabular}




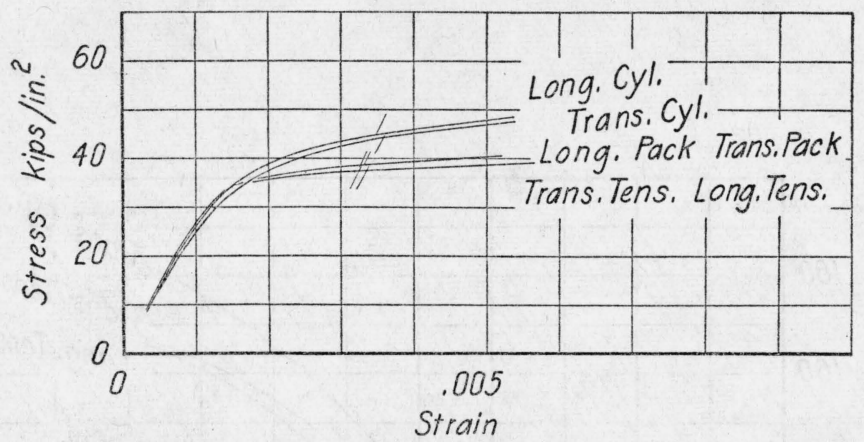

Figure 21.-17-7, 0.04-inch sheet, annealed.

Yield strength, offiset $=0.2$ percent

\begin{tabular}{|c|c|c|}
\hline Test & Longitudinal & Transverse \\
\hline $\begin{array}{l}\text { Tensile } \\
\text { Pack compressive } \\
\text { Cylinder compressive }\end{array}$ & $\begin{array}{r}\text { Kips/in.2 } \\
37.7 \\
38.8 \\
45.0\end{array}$ & $\begin{array}{r}\text { Kips/in. }{ }^{2} \\
37.9 \\
38.5 \\
44.1\end{array}$ \\
\hline
\end{tabular}

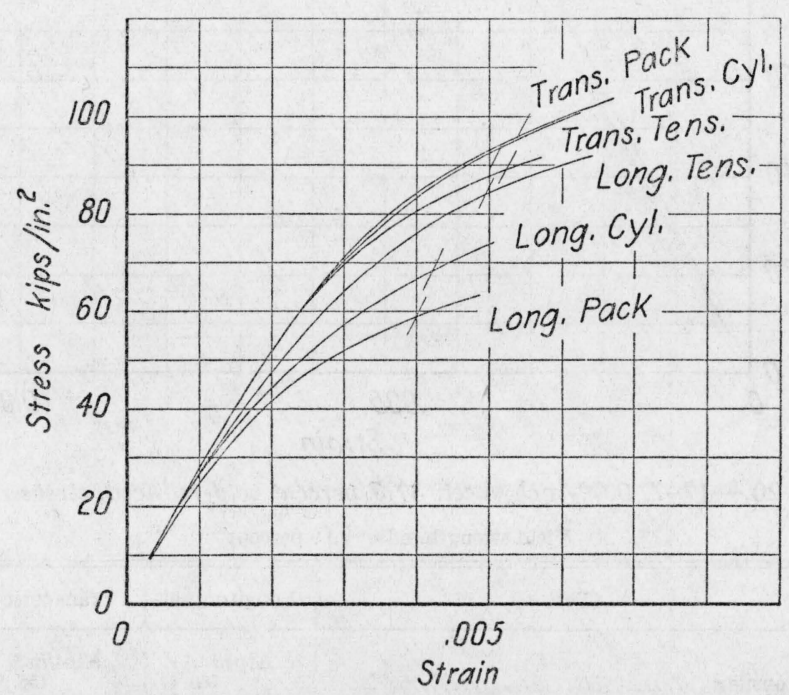

Figure 22.-17-7, 0.04-inch sheet, 9.2 percent cold-reduced.

Yield strength, ofiset $=0.2$ percent

\begin{tabular}{|c|c|c|}
\hline Test & Longitudinal & Transverse \\
\hline $\begin{array}{l}\text { Tensile } \\
\text { Pack compressive } \\
\text { Cylinder compressive. }\end{array}$ & $\begin{array}{r}\text { Kips/in. } .^{2} \\
83.9 \\
59.1 \\
68.6\end{array}$ & $\begin{array}{r}\text { Kips/in } .^{2} \\
89.2 \\
96.1 \\
94.1\end{array}$ \\
\hline
\end{tabular}




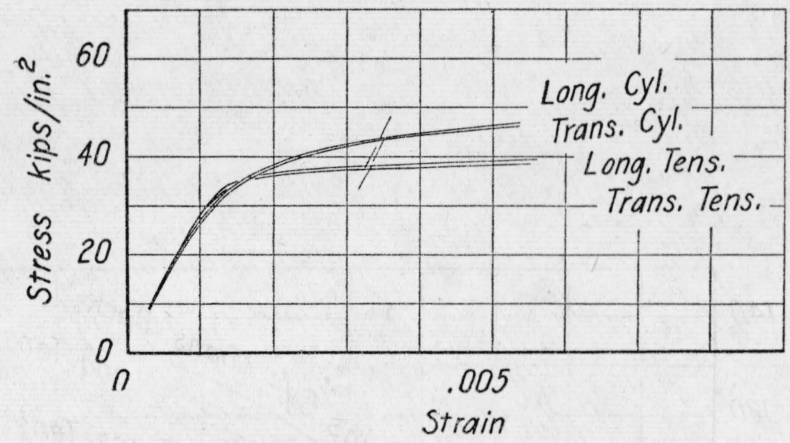

Figure 23.-17-7, 0.04-inch sheet, annealed, stress-relieved.

Yield strength, offset $=0.2$ percent

\begin{tabular}{|c|c|c|}
\hline Test & Longitudinal & Transverse \\
\hline $\begin{array}{l}\text { Tensile } \\
\text { Cylinder compressive }\end{array}$ & $\begin{array}{r}\text { Kips/in. }{ }^{2} \\
38.1 \\
43.8\end{array}$ & $\begin{array}{r}\text { Kips/in. } .^{2} \\
37.5 \\
43.4\end{array}$ \\
\hline
\end{tabular}

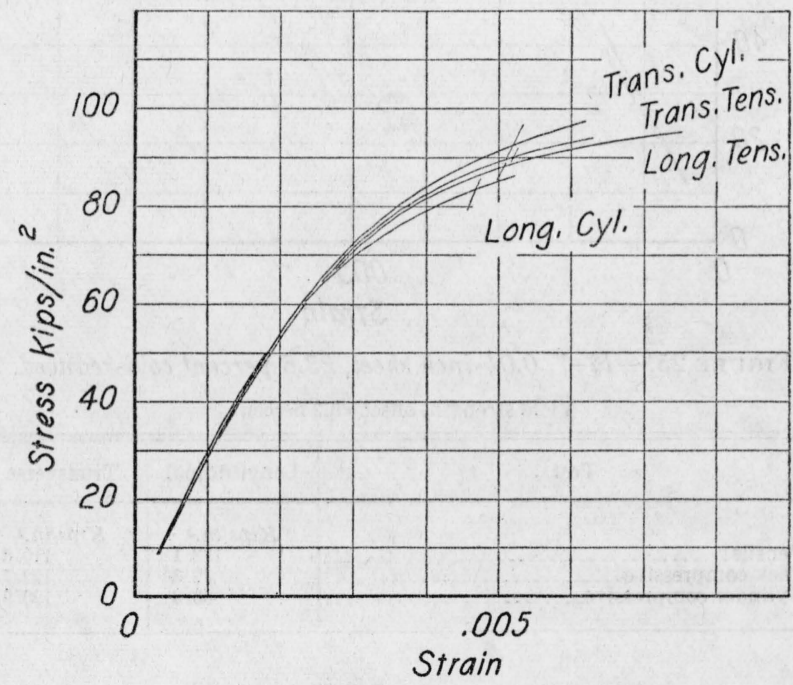

FiguRE 24.-17-7, 0.04-inch sheet, 9.2 percent cold-reduced, stress-relieved.

Yield strength, offset $=0.2$ percent

\begin{tabular}{|c|c|c|}
\hline Test & Longitudinal & Transverse \\
\hline $\begin{array}{l}\text { Tensile } \\
\text { Cylinder compressive }\end{array}$ & $\begin{array}{r}\text { Kips/in.2 } \\
88.3 \\
83.7\end{array}$ & $\begin{array}{r}\text { Kips/in. }{ }^{2} \\
90.3 \\
92.6\end{array}$ \\
\hline
\end{tabular}




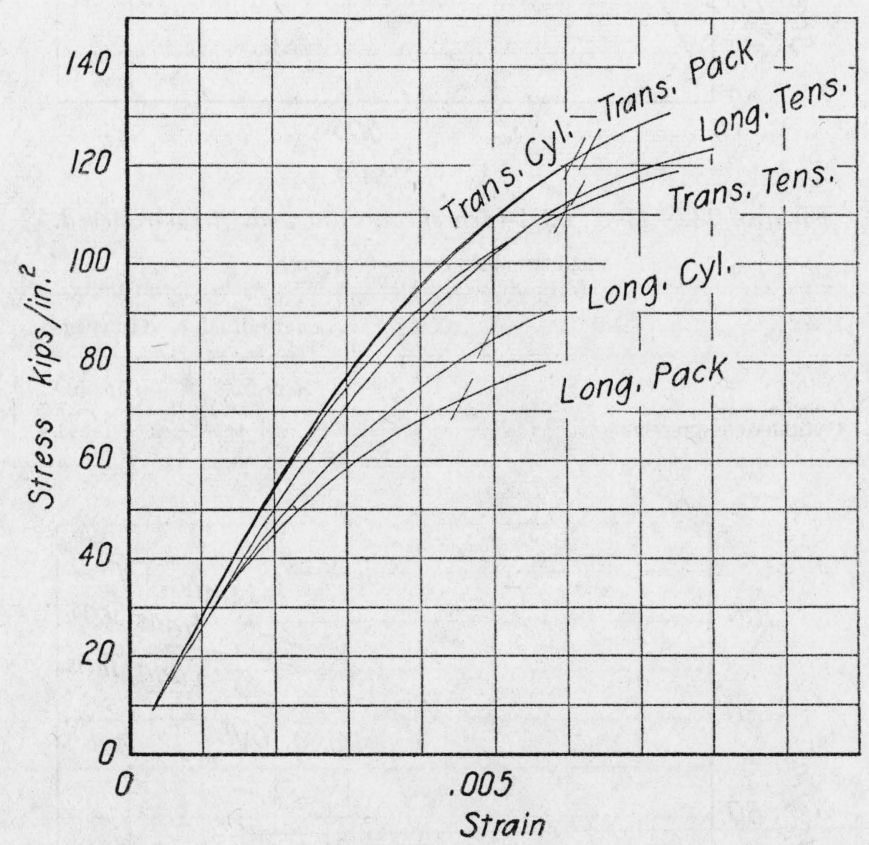

Figure 25.-17-7, 0.04-inch sheet, 23.6 percent cold-reduced.

Yield strength, offset $=0.2$ percent

\begin{tabular}{|c|c|c|}
\hline Test & Longitudinal & Transverse \\
\hline $\begin{array}{l}\text { Tensile } \\
\text { Pack compressive } \\
\text { Cylinder compressive }\end{array}$ & $\begin{array}{r}\text { Kips/in. } \\
113.1 \\
72.3 \\
83.3\end{array}$ & $\begin{array}{r}\text { Kips/in.2 } \\
110.6 \\
122.7 \\
120.9\end{array}$ \\
\hline
\end{tabular}




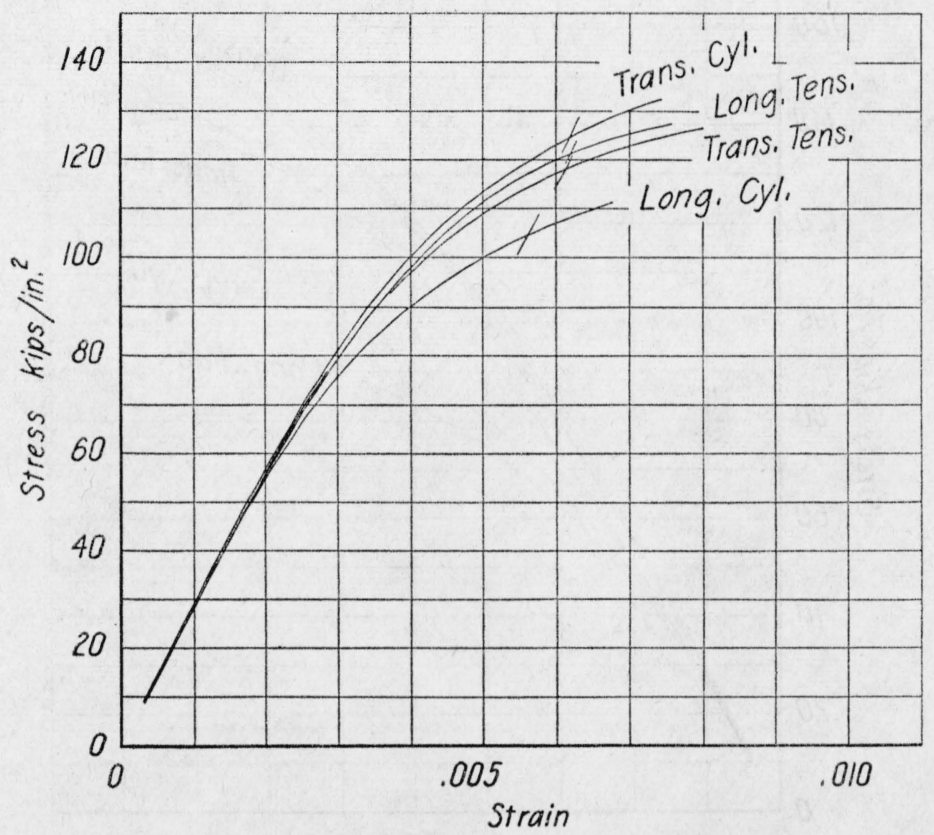

FIGURE 26.-17-7, 0.04-inch sheet, 28.6 percent cold-reduced, stress-relieved. $Y$ ield strength, offiset $=0.2$ percent

\begin{tabular}{|r|r|r|}
\hline Test & Longitudinal & Transverse \\
\cline { 2 - 3 } & Kips/in.2. & Kips/in.2. \\
Tensile & 118.1 \\
Cylinder compressive & 124.6 \\
\hline
\end{tabular}




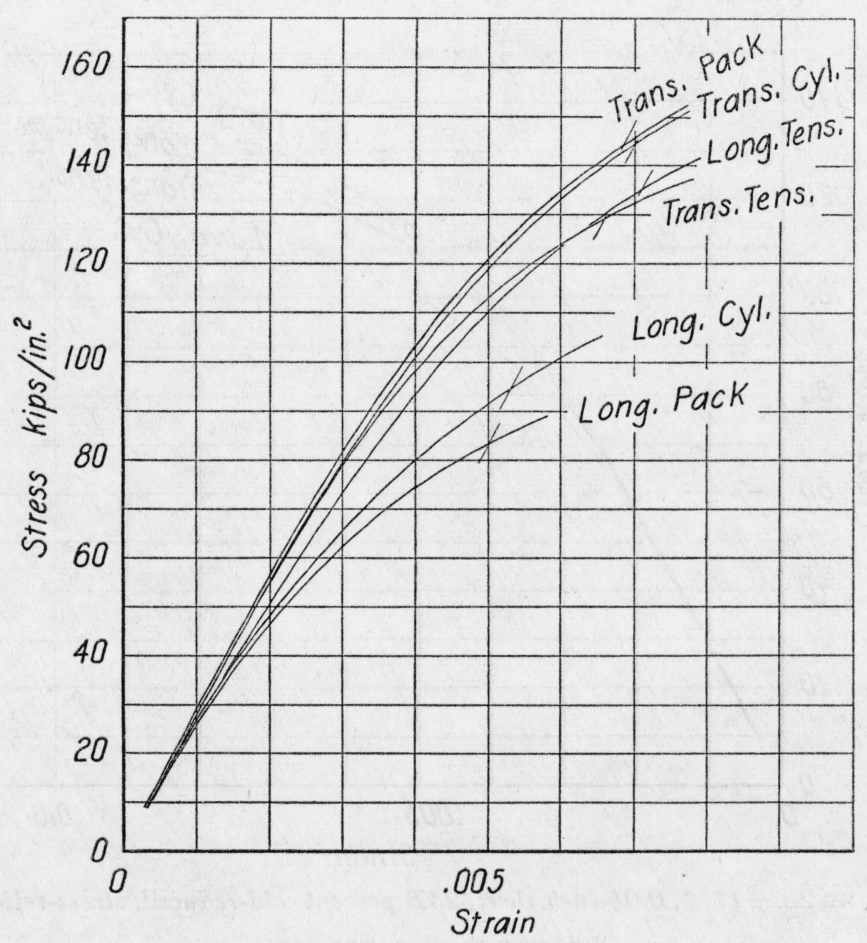

Figure 27.-17-7, 0.04-inch sheet, 28.5 percent cold-reduced.

Yield strength, offset $=0.2$ percent

\begin{tabular}{|c|c|c|}
\hline Test & Longitudinal & Transverse \\
\hline $\begin{array}{l}\text { Tensile } \\
\text { Pack compressive } \\
\text { Cylinder compressive. }\end{array}$ & $\begin{array}{r}\text { Kips/in. } .^{2} \\
135.5 \\
83.1 \\
94.8\end{array}$ & $\begin{array}{r}\text { Kips/in. }{ }^{2} \\
129.3 \\
144.5 \\
144.4\end{array}$ \\
\hline
\end{tabular}




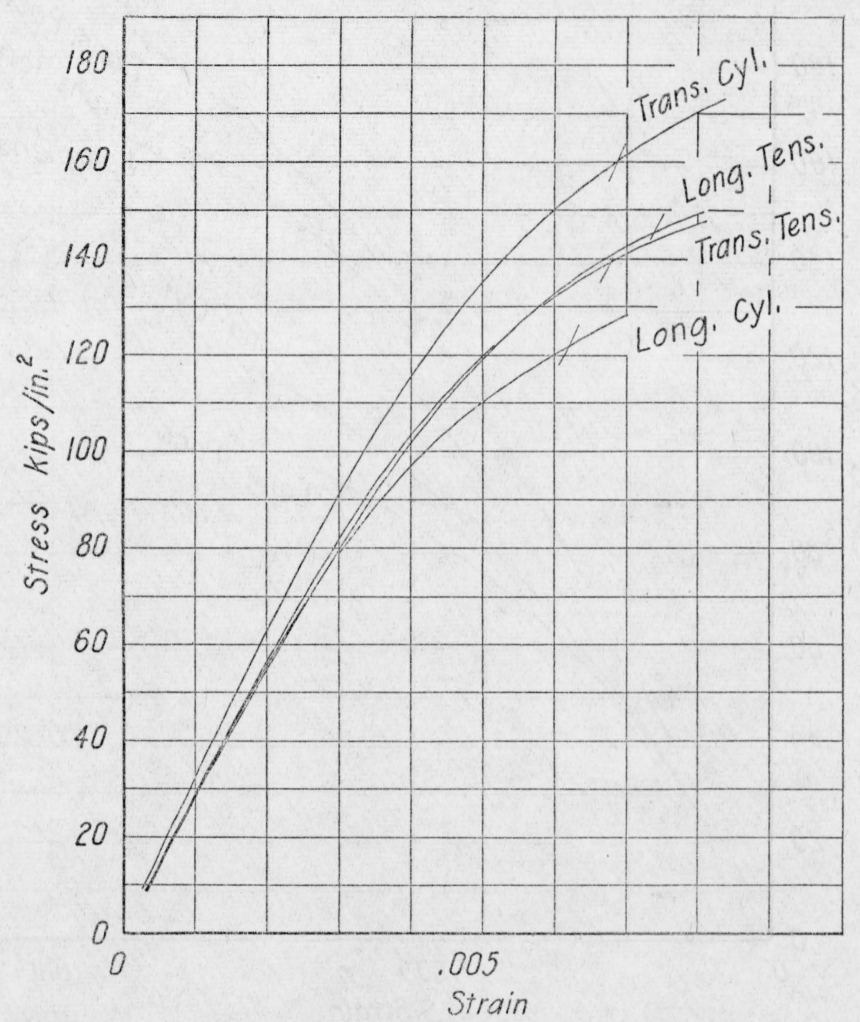

Figure 28.-17-7,0.04-inch sheet, 28.5 percent cold-reduced, stress-relieved. Yield strength, offset $=0.2$ percent

\begin{tabular}{|c|c|c|}
\hline Test & Longitudinal & Transverse \\
\hline $\begin{array}{l}\text { Tensile } \\
\text { Cylinder compressive }\end{array}$ & $\begin{array}{r}\text { Kips/in. } .^{2} \\
145.6 \\
121.7\end{array}$ & $\begin{array}{r}\text { Kips/in. }{ }^{2} \\
139.2 \\
160.4\end{array}$ \\
\hline
\end{tabular}




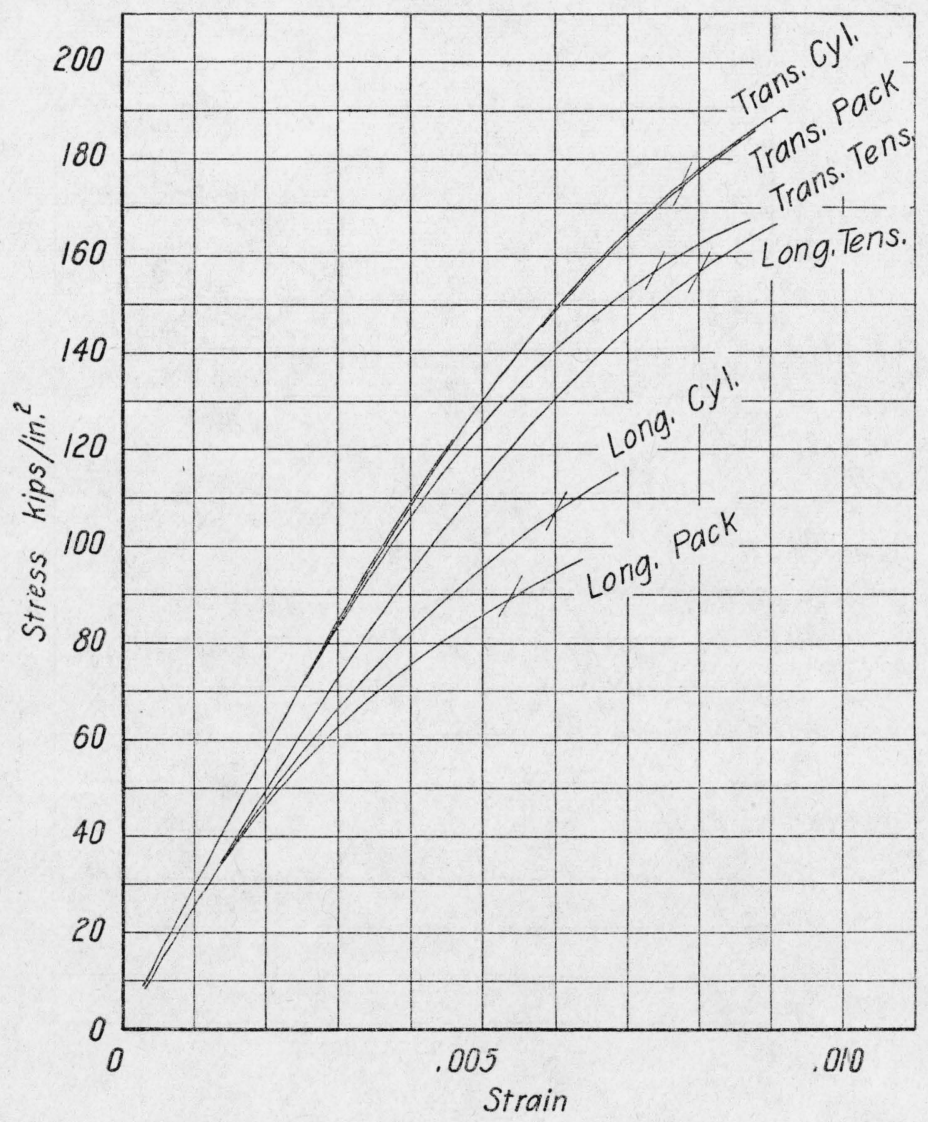

FIGURE 29.-17-7, 0.04-inch sheet, 38.8 percent cold-reduced.

Yield strength, offset $=0.2$ percent

\begin{tabular}{|c|c|c|}
\hline Test & Longitudinal & Transverse \\
\hline $\begin{array}{l}\text { Tensile } \\
\text { Pack compressive } \\
\text { Cylinder compressive }\end{array}$ & $\begin{array}{r}\text { Kips/in. } \\
156.7 \\
88.9 \\
107.3\end{array}$ & $\begin{array}{r}\text { Kips/in. }{ }^{2} \\
156.6 \\
175.1 \\
174.9\end{array}$ \\
\hline
\end{tabular}




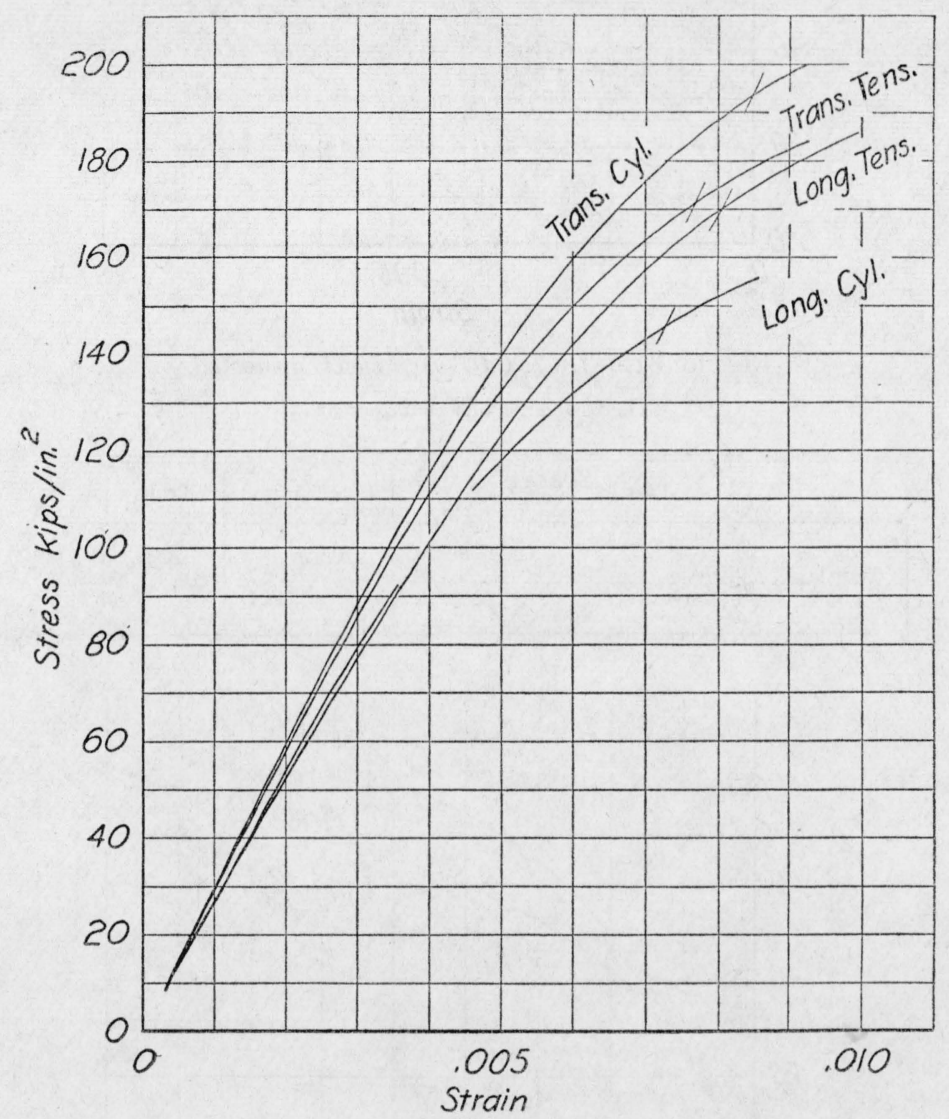

FIGURE 30.-17-7, 0.04-inch sheet, 38.8 percent cold-reduced, stress-relieved. Yield strength, offiset $=0.2$ percent

\begin{tabular}{|r|r|r|}
\hline Test & Longitudinal & Transverse \\
\cline { 2 - 3 } & Kips/in.2 & Kips/in.2 \\
Tensile & 170.0 \\
Cylinder compressive & 145.2 \\
\hline
\end{tabular}




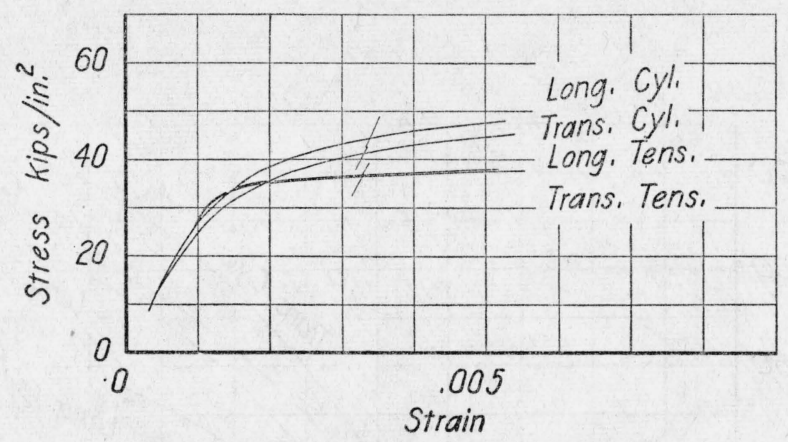

Figure 31.-18-7, 0.01-inch sheet, annealed.

Yield strength, offiset $=0.2$ percent

\begin{tabular}{|c|c|c|}
\hline Test & Longitudinal & Transverse \\
\hline $\begin{array}{l}\text { Tensile } \\
\text { Cylinder compressive }\end{array}$ & $\begin{array}{r}\text { Kips/in. } .^{2} \\
36.7 \\
44.4\end{array}$ & $\begin{array}{r}\text { Kips/in. }{ }^{2} \\
36.4 \\
41.5\end{array}$ \\
\hline
\end{tabular}

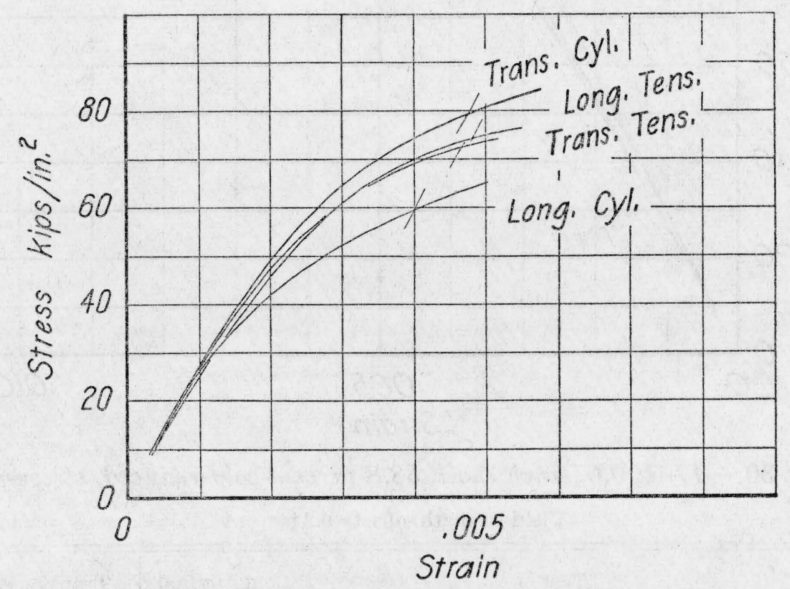

Figure 32.-18-7, 0.01-inch sheet, 12.0 percent cold-reduced.

Yield strength, offset $=0.2$ percent

\begin{tabular}{|c|c|c|}
\hline Test & Longitudinal & Transverse \\
\hline $\begin{array}{l}\text { Tensile } \\
\text { Cylinder compressive }\end{array}$ & $\begin{array}{r}\text { Kips/in. } .^{2} \\
74.3 \\
59.7\end{array}$ & $\begin{array}{r}\text { Kips/in. }{ }^{2} \\
72.5 \\
79.4\end{array}$ \\
\hline
\end{tabular}




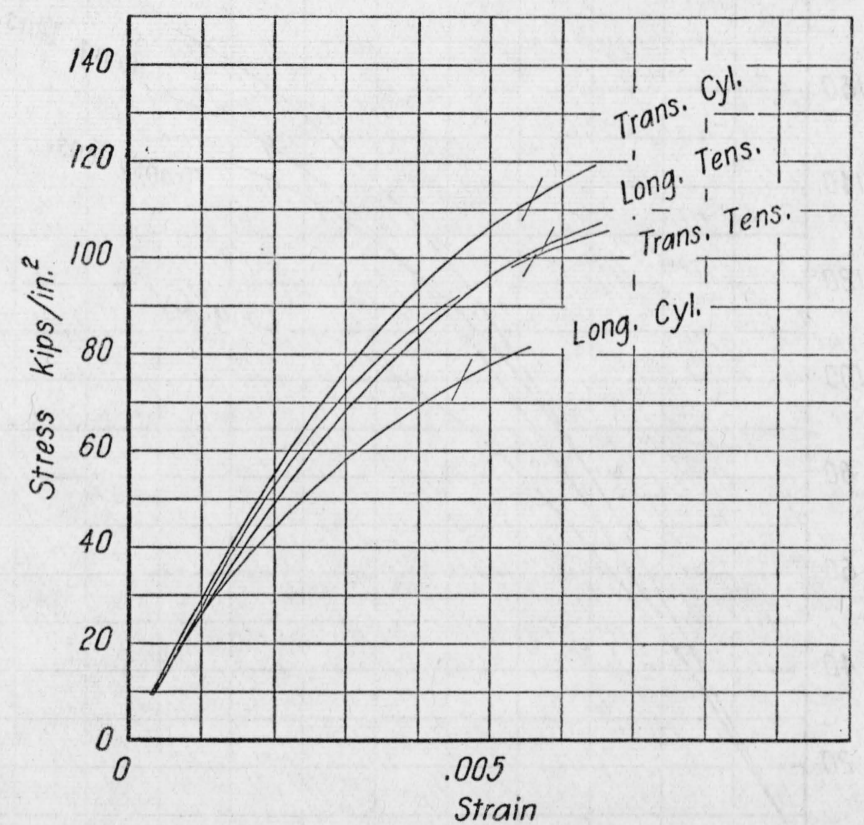

Figure 33.-18-7, 0.01-inch sheet, 19.6 percent cold-reduced. Yield strength, offset $=0.2$ percent

\begin{tabular}{|c|c|c|}
\hline Test & Longitudinal & Transverse \\
\hline $\begin{array}{l}\text { Tensile } \\
\text { Cylinder compressive }\end{array}$ & $\begin{array}{r}\text { Kips/in. } .^{2} \\
102.6 \\
74.5\end{array}$ & $\begin{array}{r}\text { Kips/in. }{ }^{2} \\
100.2 \\
112.1\end{array}$ \\
\hline
\end{tabular}




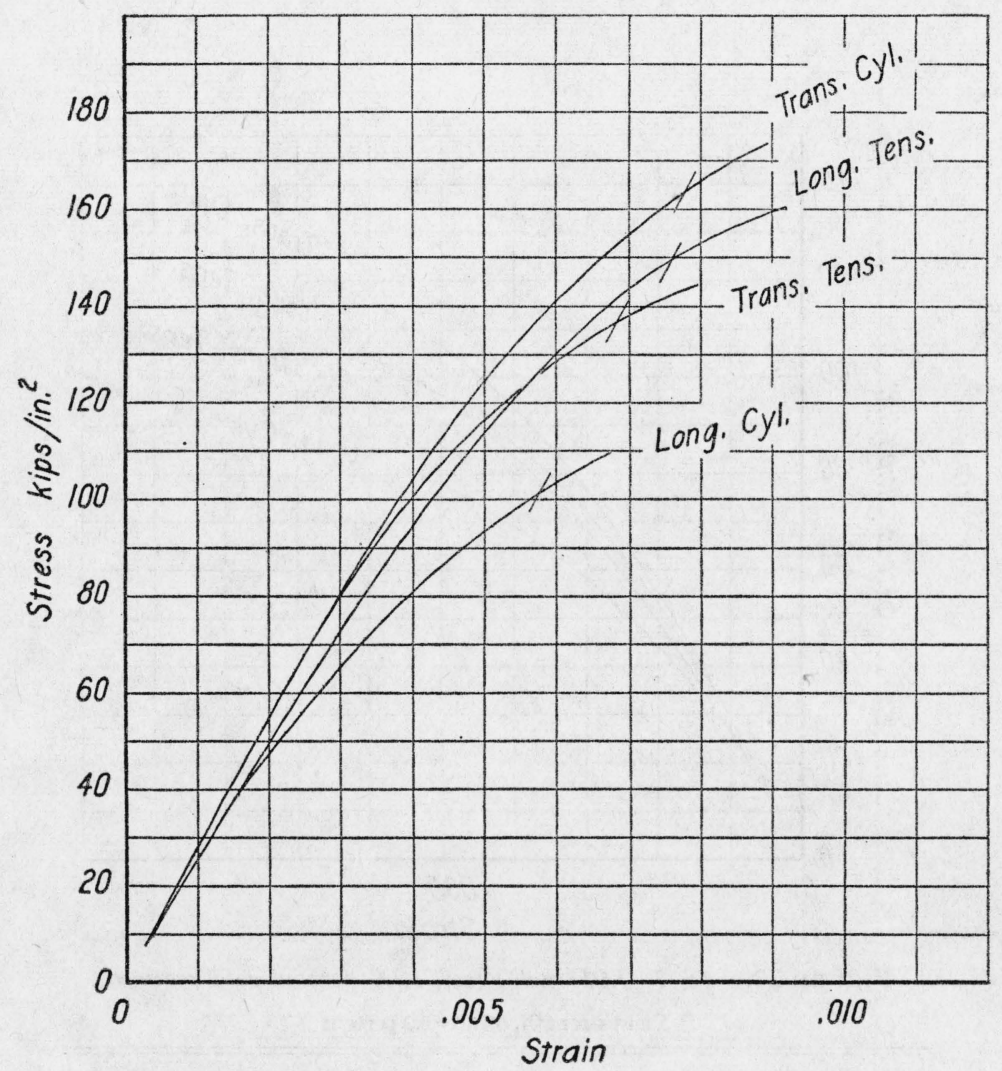

FiguRE 34.-18- $7,0.01$-inch sheet, 31.5 percent cold-reduced. Yield strength, offset $=0.2$ percent

\begin{tabular}{|c|c|c|}
\hline Test & Longitudinal & Transverse \\
\hline $\begin{array}{l}\text { Tensile } \\
\text { Cylinder compressive }\end{array}$ & $\begin{array}{r}\text { Kips/in.2 } \\
149.7 \\
100.9\end{array}$ & $\begin{array}{r}\text { Kips/in.2 } \\
136.7 \\
163.8\end{array}$ \\
\hline
\end{tabular}




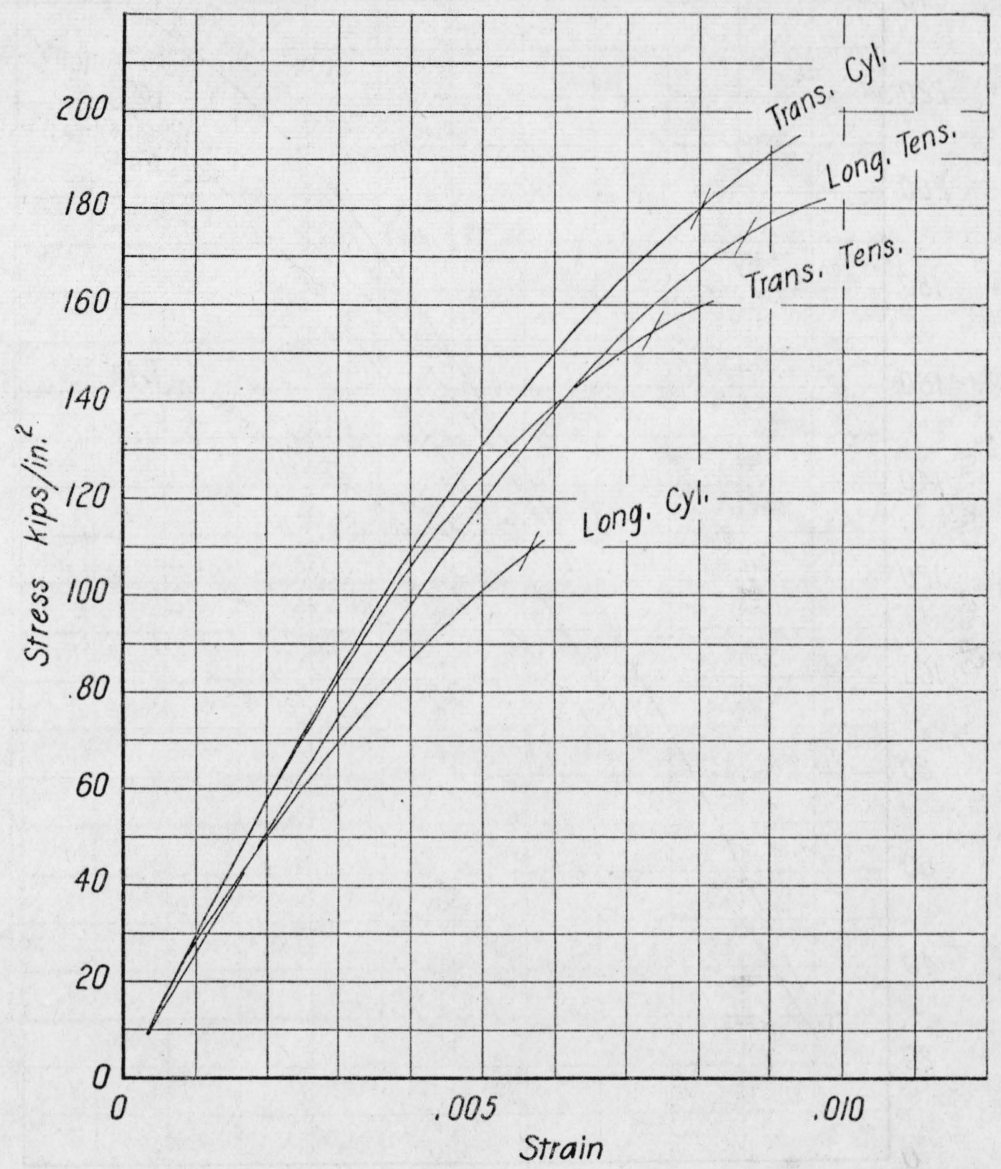

FiguRE 35.-18- $\%$, 0.01-inch sheet, 40.0 percent cold-reduced.

Yield strength, 'offset $=0.2$ percent

\begin{tabular}{|c|c|c|}
\hline Test & Longitudinal & Transverse \\
\hline $\begin{array}{l}\text { Tensile } \\
\text { Cylinder compressive }\end{array}$ & $\begin{array}{r}\text { Kips/in.2 } \\
174.5 \\
109.0\end{array}$ & $\begin{array}{r}\text { Kips/in. } .^{2} \\
154.4 \\
180.7\end{array}$ \\
\hline
\end{tabular}




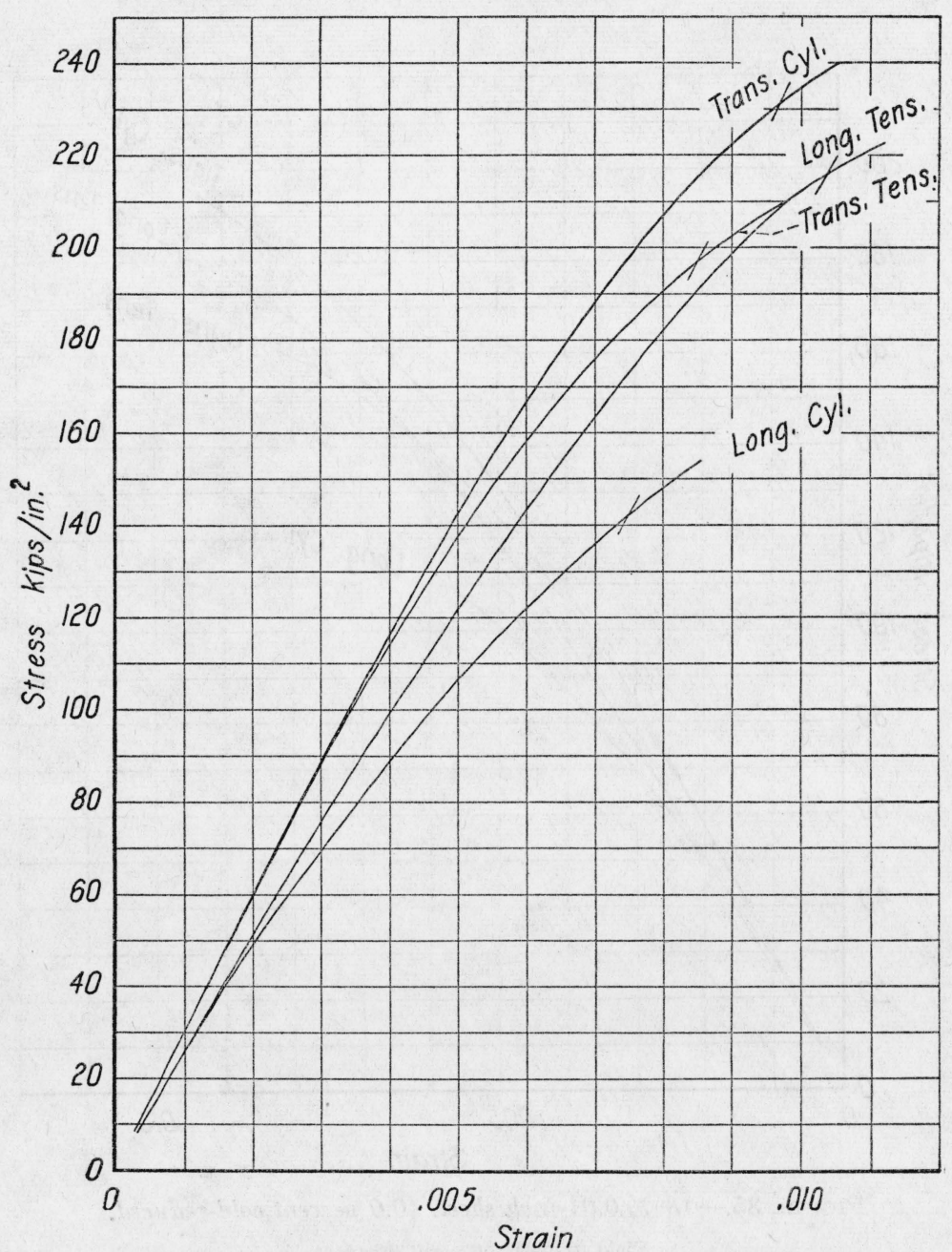

Figure 36.-18-7, 0.01-inch sheet, 50.0 percent cold-reduced.

Yield strength, ofiset $=0.2$ percent

\begin{tabular}{|c|c|c|}
\hline Test & Longitudinal & Transverse \\
\hline $\begin{array}{l}\text { Tensile } \\
\text { Cylinder compressive }\end{array}$ & $\begin{array}{r}\text { Kips/in. } .^{2} \\
216.0 \\
142.2\end{array}$ & $\begin{array}{r}\text { Kips/in.2 } \\
197.0 \\
231.6\end{array}$ \\
\hline
\end{tabular}




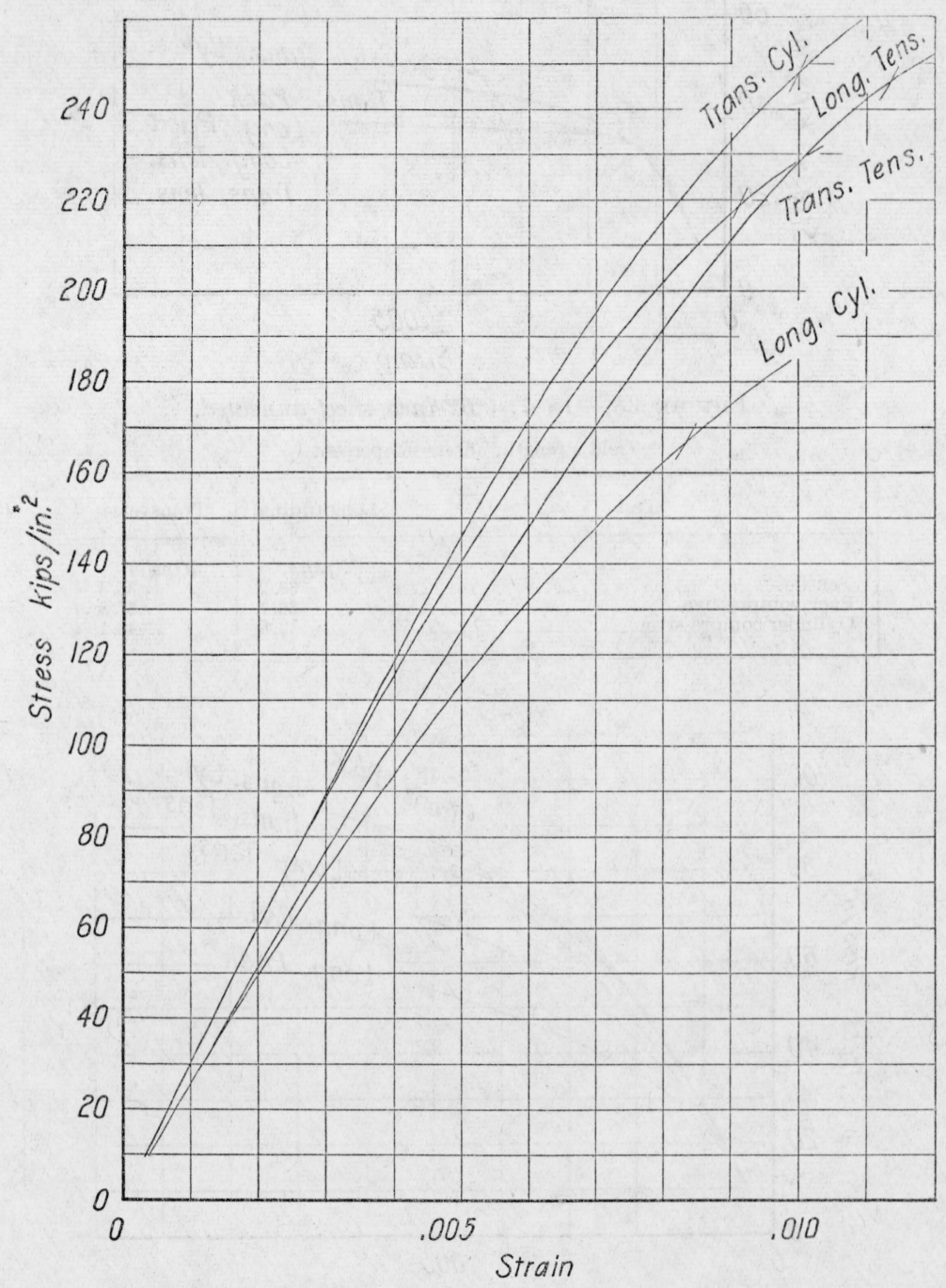

Figure 37.-18- $\%$, 0.01-inch sheet, 52.5 percent cold-reduced.

Yield strength, offiset $=0.2$ percent

\begin{tabular}{|c|c|c|}
\hline Test & Longitudinal & Transverse \\
\hline $\begin{array}{l}\text { Tensile } \\
\text { Cylinder compressive }\end{array}$ & $\begin{array}{r}\text { Kips/in. } .^{2} \\
246.4 \\
166.8\end{array}$ & $\begin{array}{r}\text { Kips/in } .^{2} \\
218.5 \\
248.2\end{array}$ \\
\hline
\end{tabular}




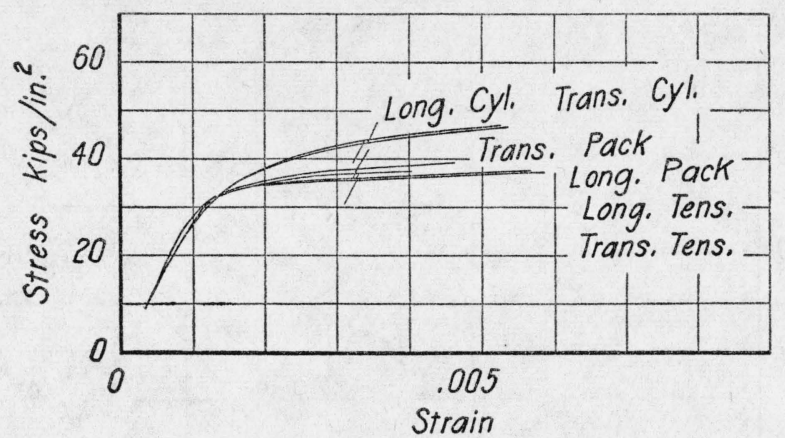

Figure 38.-18-7, 0.02-inch sheet-annealed.

Yield strength, offset $=0.2$ percent

\begin{tabular}{|c|c|c|}
\hline Test & Longitudinal & Transverse \\
\hline $\begin{array}{l}\text { Tensile } \\
\text { Pack compressive } \\
\text { Cylinder compressive }\end{array}$ & $\begin{array}{r}\text { Kips/in. } .^{2} \\
36.2 \\
36.7 \\
43.8\end{array}$ & $\begin{array}{r}\text { Kips/in. }{ }^{2} \\
35.8 \\
37.8 \\
43.1\end{array}$ \\
\hline
\end{tabular}

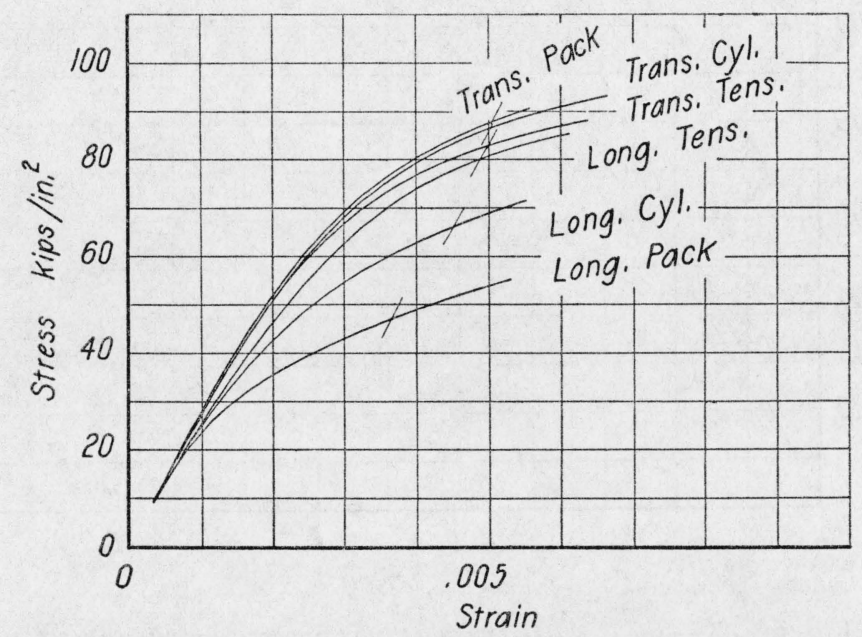

FiguRE 39.-18-7, 0.02-inch sheet, 5.3 percent cold-reduced.

Yield strength, offset $=0.2$ percent

\begin{tabular}{|c|r|r|}
\hline Test & Longitudinal & Transverse \\
\cline { 2 - 4 } & Kips/in.2 & Kips/in.2 \\
Tensile_... & 80.4 & 82.9 \\
Pack compressive & 47.4 & 88.1 \\
Cylinder compressive & 66.8 & 86.6 \\
\hline
\end{tabular}




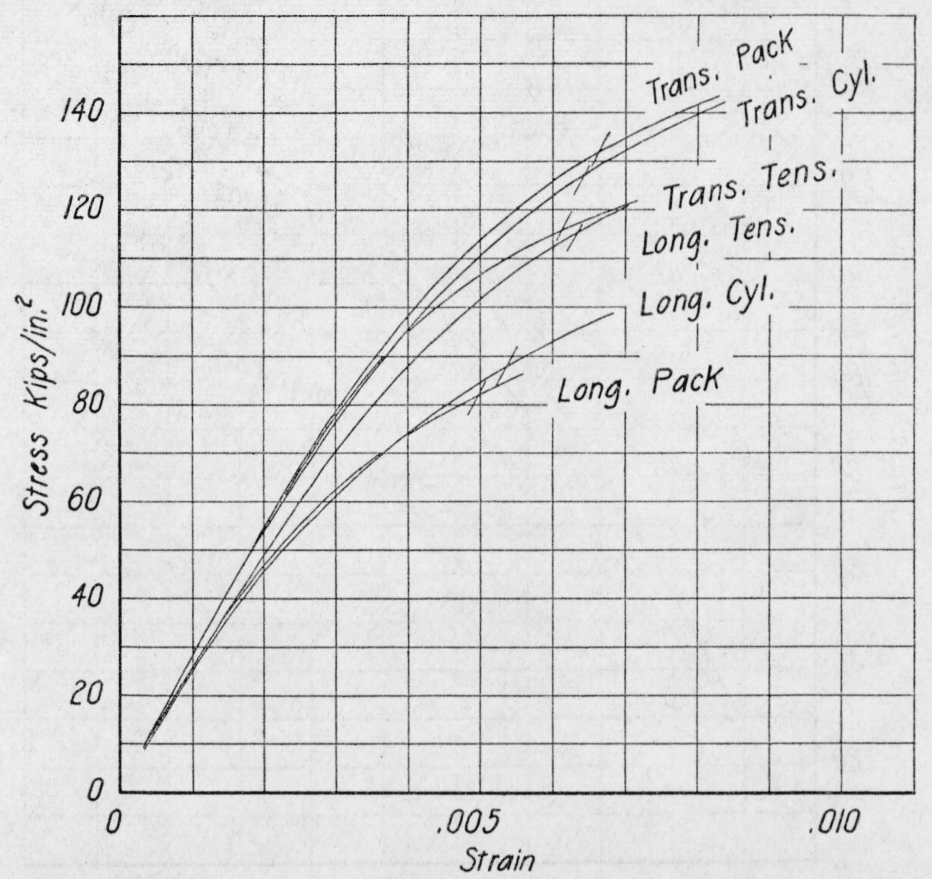

Figure 40.-18-7, 0.02-inch sheet, 18.3 percent cold-reduced. Yield strength, offset $=0.2$ percent

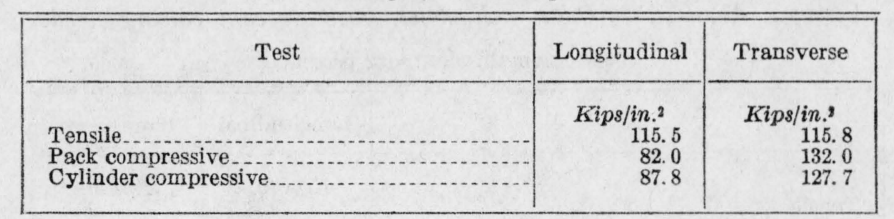




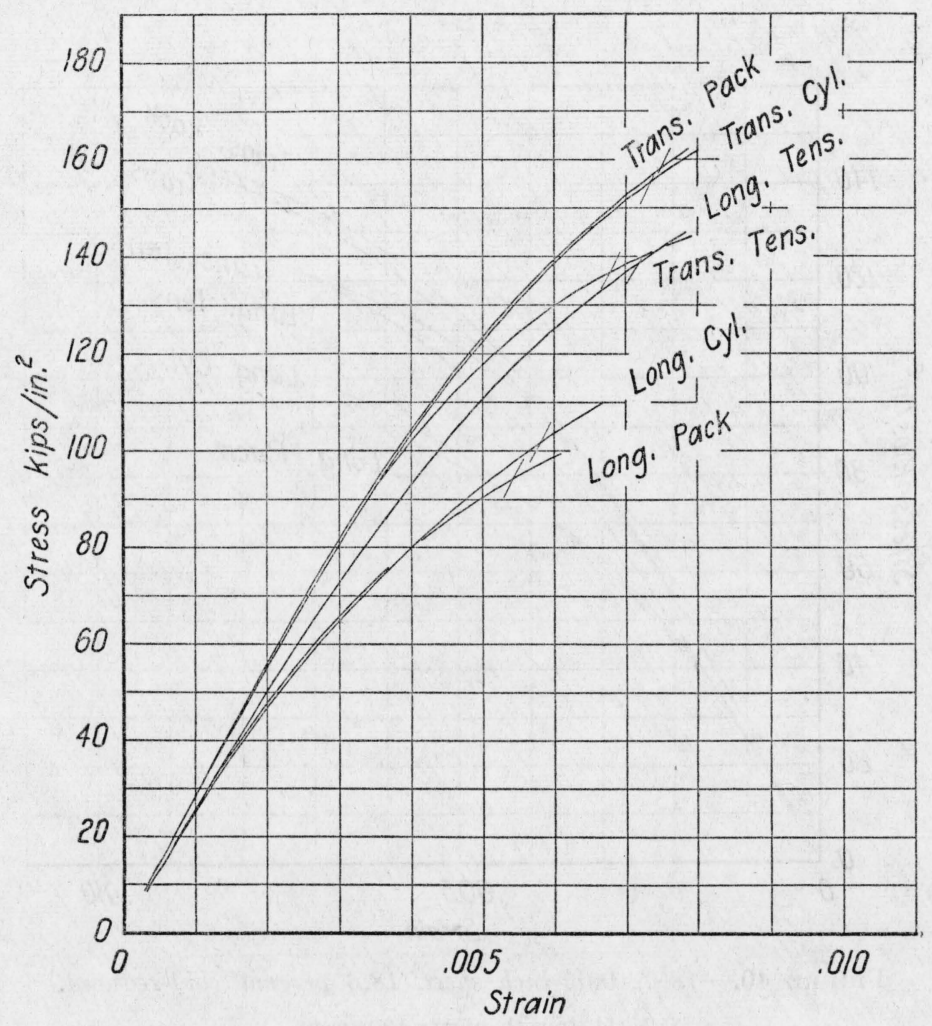

Figure 41.-18-7, 0.02-inch sheet, 29.8 percent cold-reduced.

Yield strength, offset $=0.2$ percent

\begin{tabular}{|c|c|c|}
\hline Test & Longitudinal & Transverse \\
\hline $\begin{array}{l}\text { Tensile } \\
\text { Pack compressive } \\
\text { Cylinder compressive }\end{array}$ & $\begin{array}{r}\text { Kips/in. } .^{2} \\
138.7 \\
94.3 \\
101.7\end{array}$ & $\begin{array}{r}\text { Kips/in. }{ }^{2} \\
136.6 \\
158.1 \\
155.3\end{array}$ \\
\hline
\end{tabular}




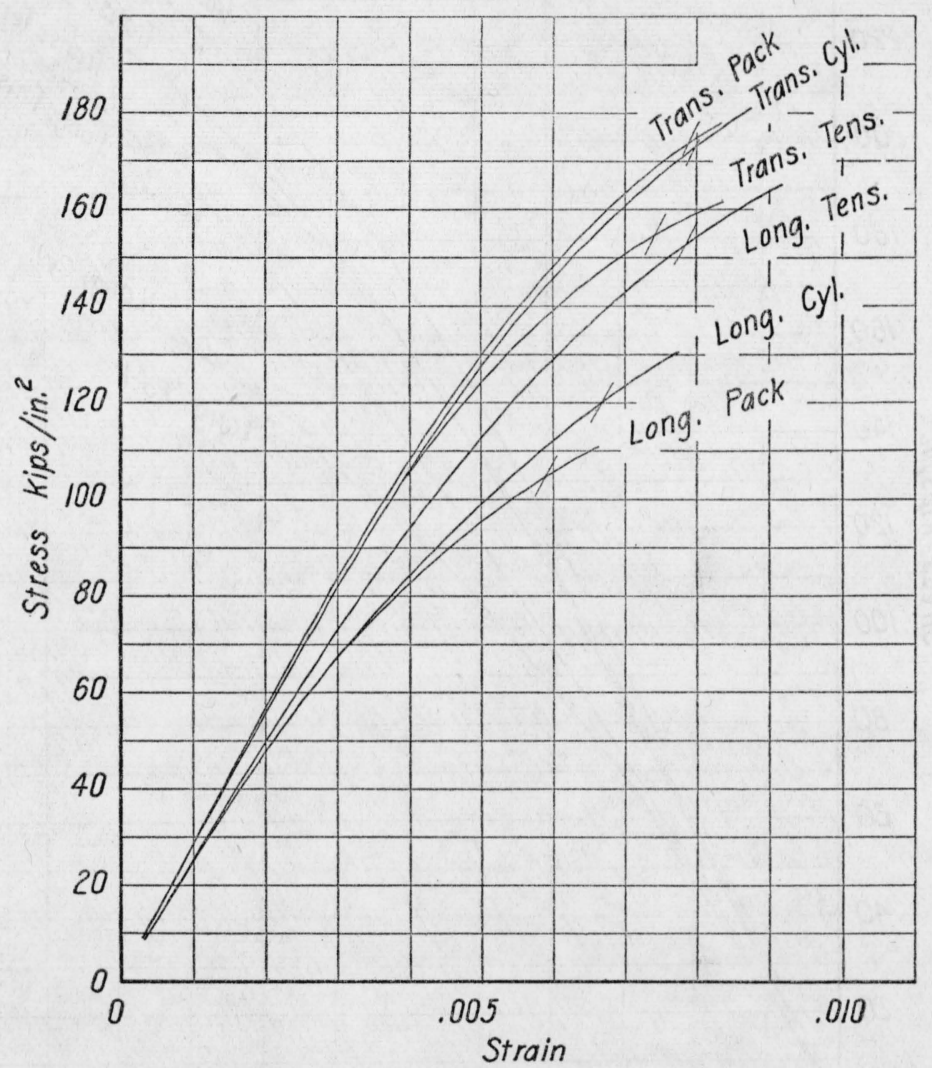

Figure 42.-18-7, 0.02-inch sheet, 37.5 percent cold-reduced.

Yield strength, offset $=0.2$ percent

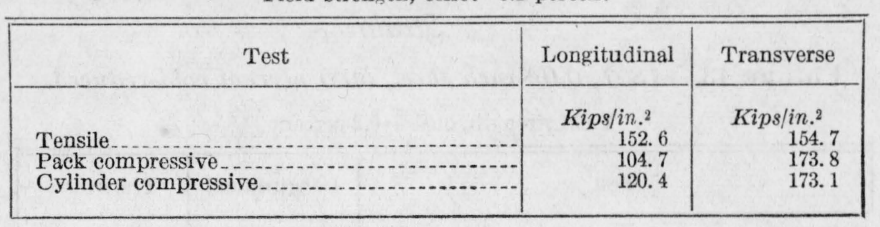




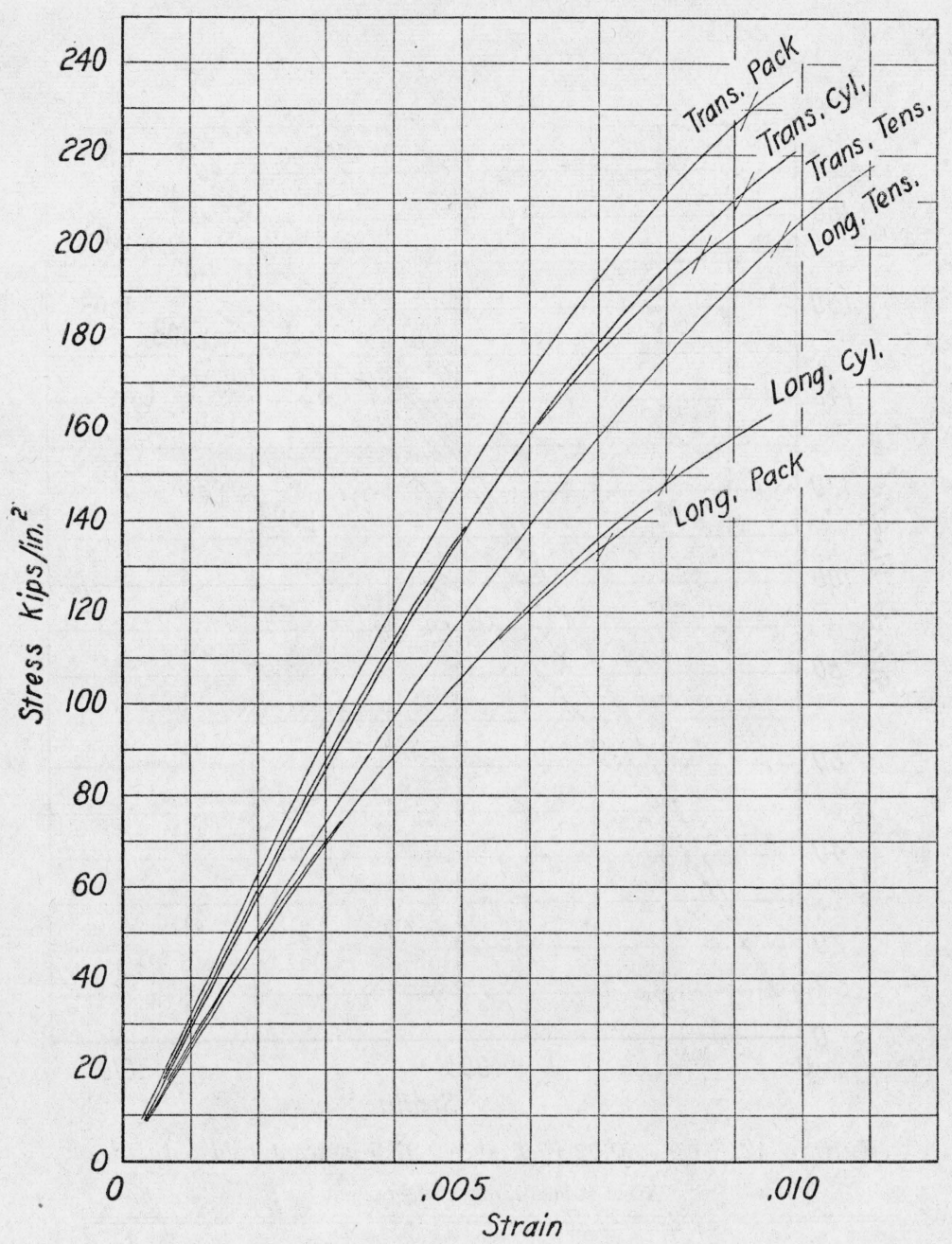

FiguRE 43.-18- $\gamma$, 0.02-inch sheet, 50.0 percent cold-reduced. Yield strength, offset $=0.2$ percent

\begin{tabular}{|c|c|c|}
\hline Test & Longitudinal & Transverse \\
\hline $\begin{array}{l}\text { Tensile } \\
\text { Pack compressive } \\
\text { Cylinder compressive }\end{array}$ & $\begin{array}{r}\text { Kips/in } .^{2} \\
201.6 \\
135.1 \\
147.8\end{array}$ & $\begin{array}{r}\text { Kips/in.2 } \\
198.3 \\
228.8 \\
212.5\end{array}$ \\
\hline
\end{tabular}




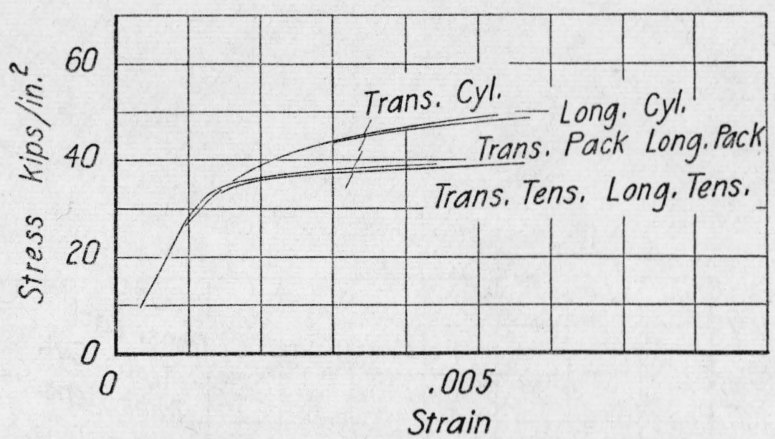

Figure 44.-18- $\%$, 0.035-inch sheet, annealed.

Yield strength, offset $=0.2$ percent

\begin{tabular}{|r|r|r|}
\hline Test & Longitudinal & Transverse \\
\cline { 2 - 3 } & Kips/in. ${ }^{2}$ & Kips/in. ${ }^{2}$ \\
Tensile & 37.5 & 37.7 \\
Pack compressive & 37.8 & 38.2 \\
Cylinder compressive & 45.1 & 45.4 \\
\hline
\end{tabular}

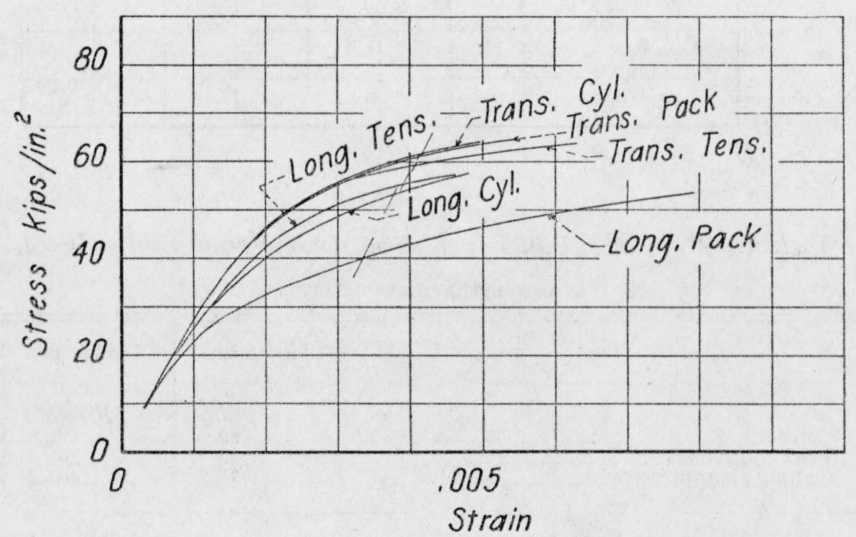

FIGURE 45.-18- $7,0.085$-inch sheet, 7.5 percent cold-reduced.

Yield strength, offset $=0.2$ percent

\begin{tabular}{|c|r|r|}
\hline Test & Longitudinal & Transverse \\
\hline & Kips $/$ in.2. & Kips $/$ in $^{2}{ }^{2}$ \\
Tensile & 55.4 & 59.1 \\
Pack compressive & 40.1 & 61.0 \\
Cylinder compressive & 53.2 & 61.3 \\
\hline
\end{tabular}




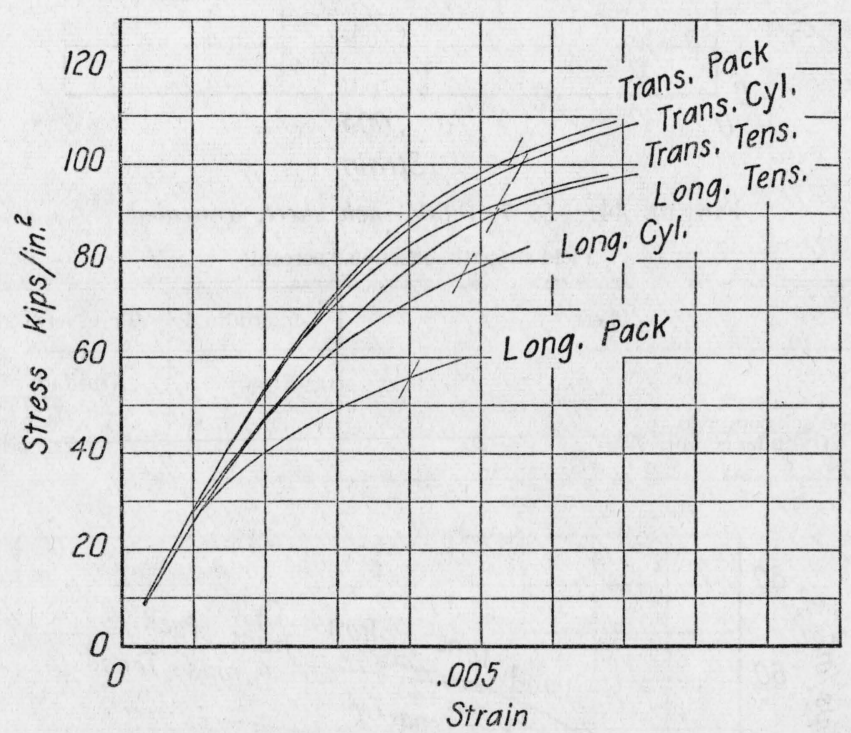

FIguRE 46.-18- $7,0.035$-inch sheet, 19.6 percent cold-reduced.

Yield strength, offset $=0.2$ percent

\begin{tabular}{|c|c|c|}
\hline Test & Longitudinal & Transverse \\
\hline $\begin{array}{l}\text { Tensile } \\
\text { Pack compressive } \\
\text { Cylinder compressive }\end{array}$ & $\begin{array}{c}\text { Kips/in. } .^{2} \\
89.5 \\
55.9 \\
77.3\end{array}$ & $\begin{array}{r}\text { Kips/in } .^{2} \\
91.8 \\
101.2 \\
100.2\end{array}$ \\
\hline
\end{tabular}




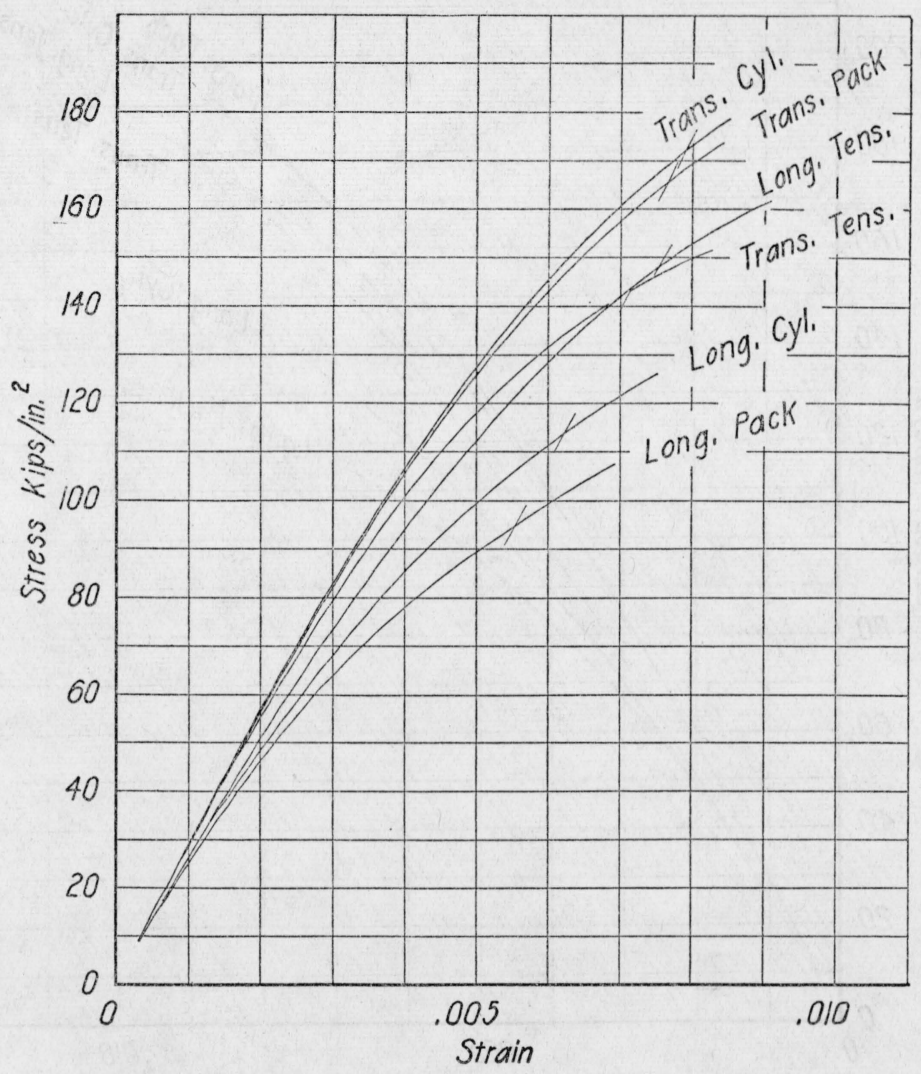

Figure 47.-18-7, 0.035-inch sheet, 29.2 percent cold-reduced.

Yield strength, offset $=0.2$ percent

\begin{tabular}{|r|r|r|}
\hline Test & Longitudinal & Transverse \\
\hline & Kips $/$ in. $^{2}$ & Kips $/$ in.$^{2}$ \\
Tensile & 148.2 & 143.0 \\
Pack compressive & 94.8 & 165.6 \\
Cylinder compressive & 113.5 & 172.1 \\
\hline
\end{tabular}




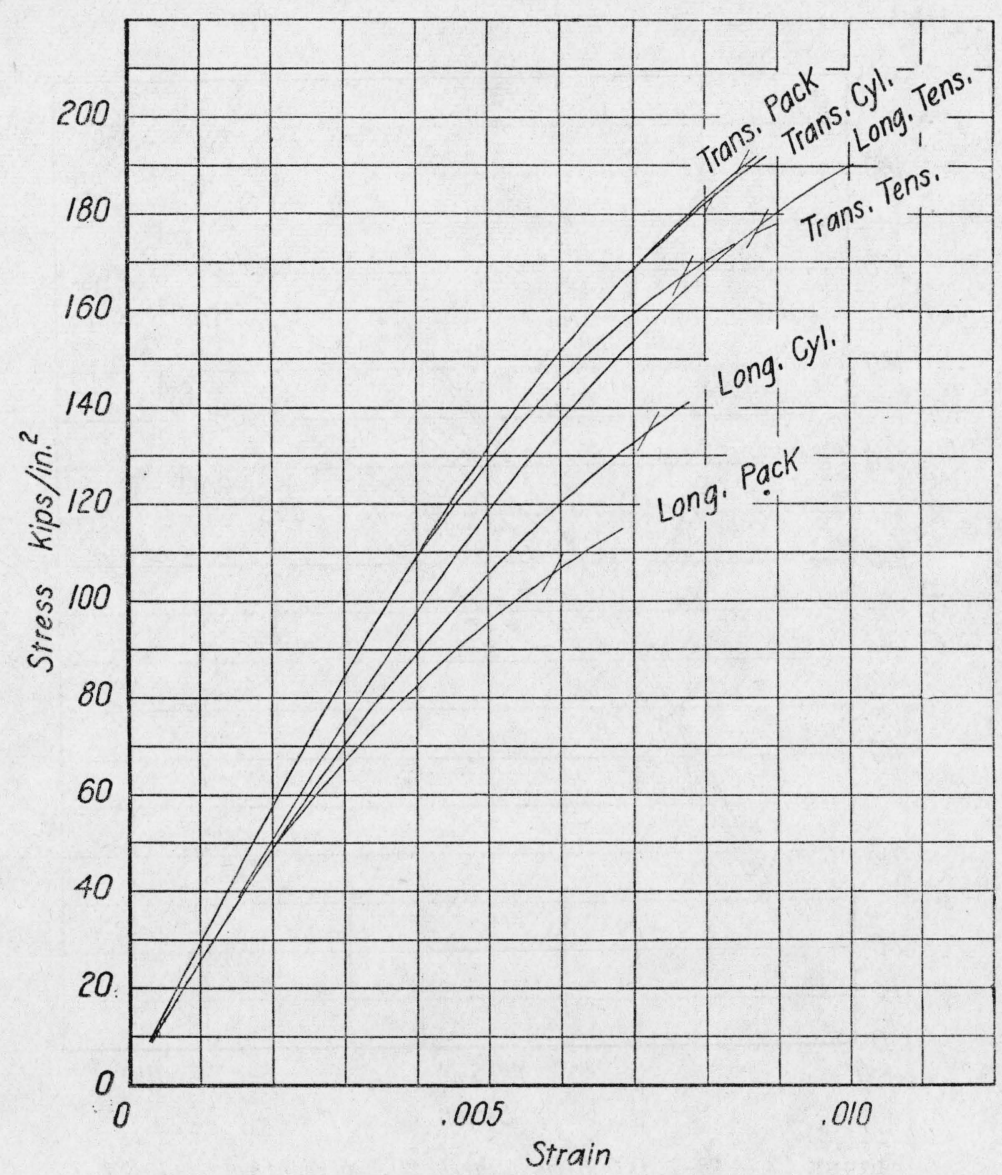

FIGURE 48.-18-7, 0.035-inch sheet, 39.7 percent cold-reduced.

Yield strength, offset $=0.2$ percent

\begin{tabular}{|r|r|r|}
\hline Test & Longitudinal & Transverse \\
\cline { 2 - 3 } & Kips/in.2. & Kips/in.2 \\
Tensile & 177.7 & 167.0 \\
Pack compressive & 105.5 & 189.1 \\
Cylinder compressive & 135.0 & 183.8 \\
\hline
\end{tabular}




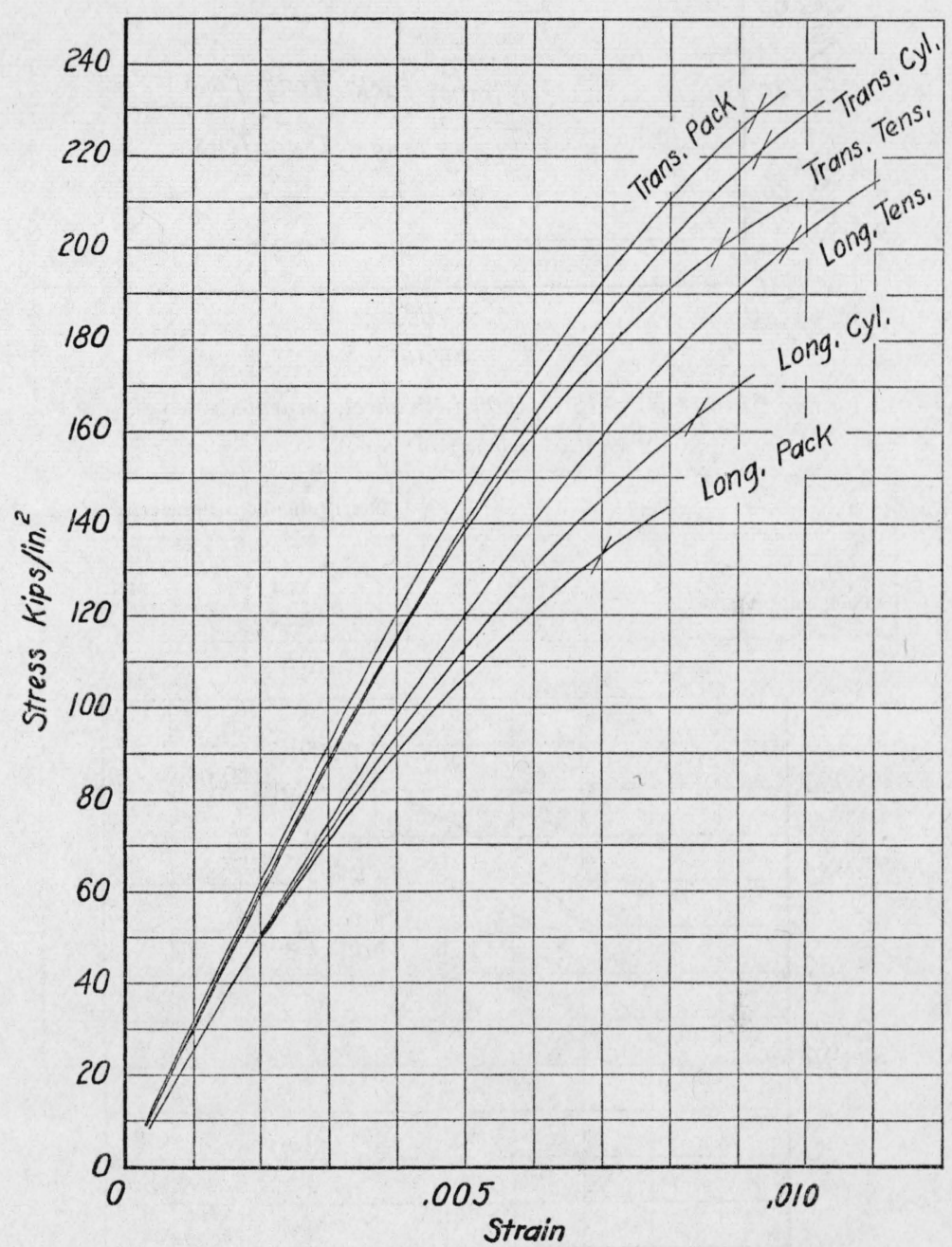

FigURE 49.-18-7, 0.035-inch sheet, 47.0 percent cold-reduced.

Yield strength, offset $=0.2$ percent

\begin{tabular}{|r|r|r|}
\hline Test & Longitudinal & Transverse \\
\hline & Kips/in.2 & Kips/in.2 \\
Tensile & 200.9 & 200.4 \\
Pack compressive..... & 133.5 & 229.5 \\
Cylinder compressive & 163.2 & 221.5 \\
\hline
\end{tabular}




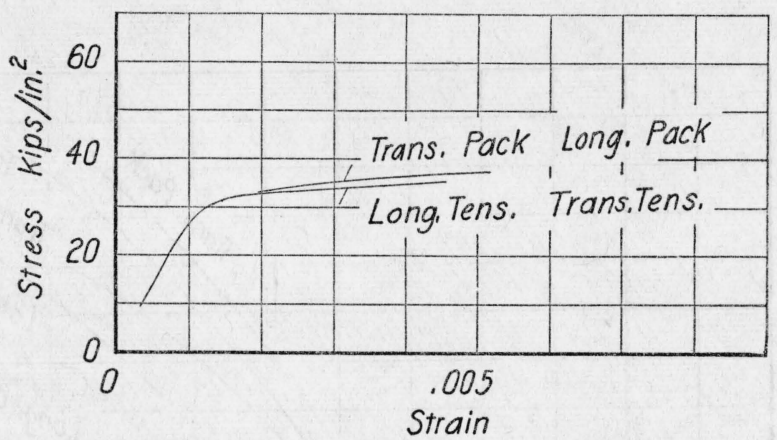

FIGURE 50.-18-7, 0.06-inch sheet, annealed.

Yield strength, offset $=0.2$ percent

\begin{tabular}{|c|c|c|}
\hline Test & Longitudinal & Transverse \\
\hline $\begin{array}{l}\text { Tensile } \\
\text { Pack compressive }\end{array}$ & $\begin{array}{r}\text { Kips/in. }{ }^{2} \\
34.1 \\
35.3\end{array}$ & $\begin{array}{r}\text { Kips/in. } .^{2} \\
34.1 \\
35.5\end{array}$ \\
\hline
\end{tabular}

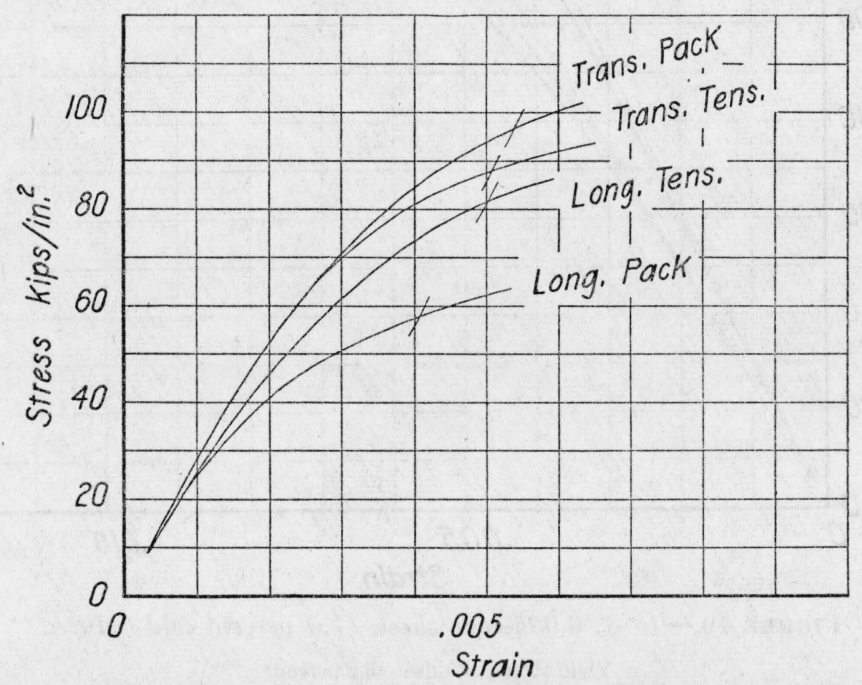

FIGURE 51.-18-7, 0.06-inch sheet, 6.7 percent cold-reduced.

Yield strength, offset $=0.2$ percent

\begin{tabular}{|c|c|c|}
\hline Test & Longitudinal & Transverse \\
\hline $\begin{array}{l}\text { Tensile } \\
\text { Pack compressive... }\end{array}$ & $\begin{array}{r}\text { Kips/in.2 } \\
81.5 \\
57.8\end{array}$ & $\begin{array}{r}\text { Kips/in. } .^{2} \\
88.3 \\
96.3\end{array}$ \\
\hline
\end{tabular}




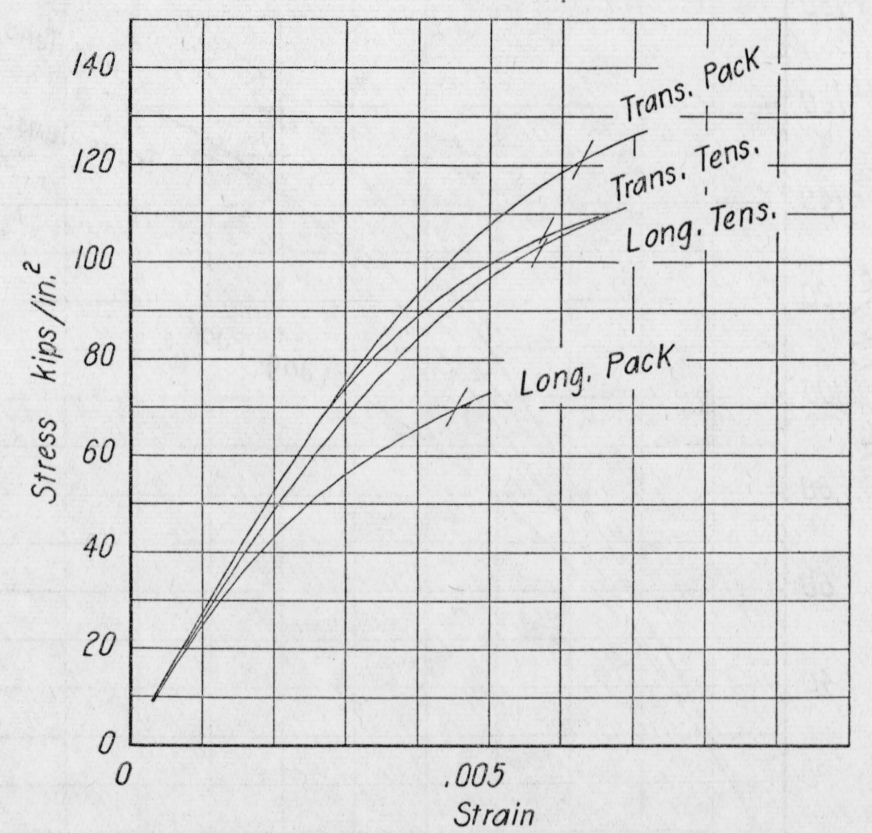

Figure 52.-18-7, 0.06-inch sheet, 18.1 percent cold-reduced.

Yield strength, offset $=0.2$ percent

\begin{tabular}{|c|c|c|}
\hline Test & Longitudinal & Transverse \\
\hline $\begin{array}{l}\text { Tensile } \\
\text { Pack compressive... }\end{array}$ & $\begin{array}{r}\text { Kips/in. } .^{2} \\
103.7 \\
69.4\end{array}$ & $\begin{array}{r}\text { Kips/in. } .^{2} \\
105.2 \\
120.7\end{array}$ \\
\hline
\end{tabular}




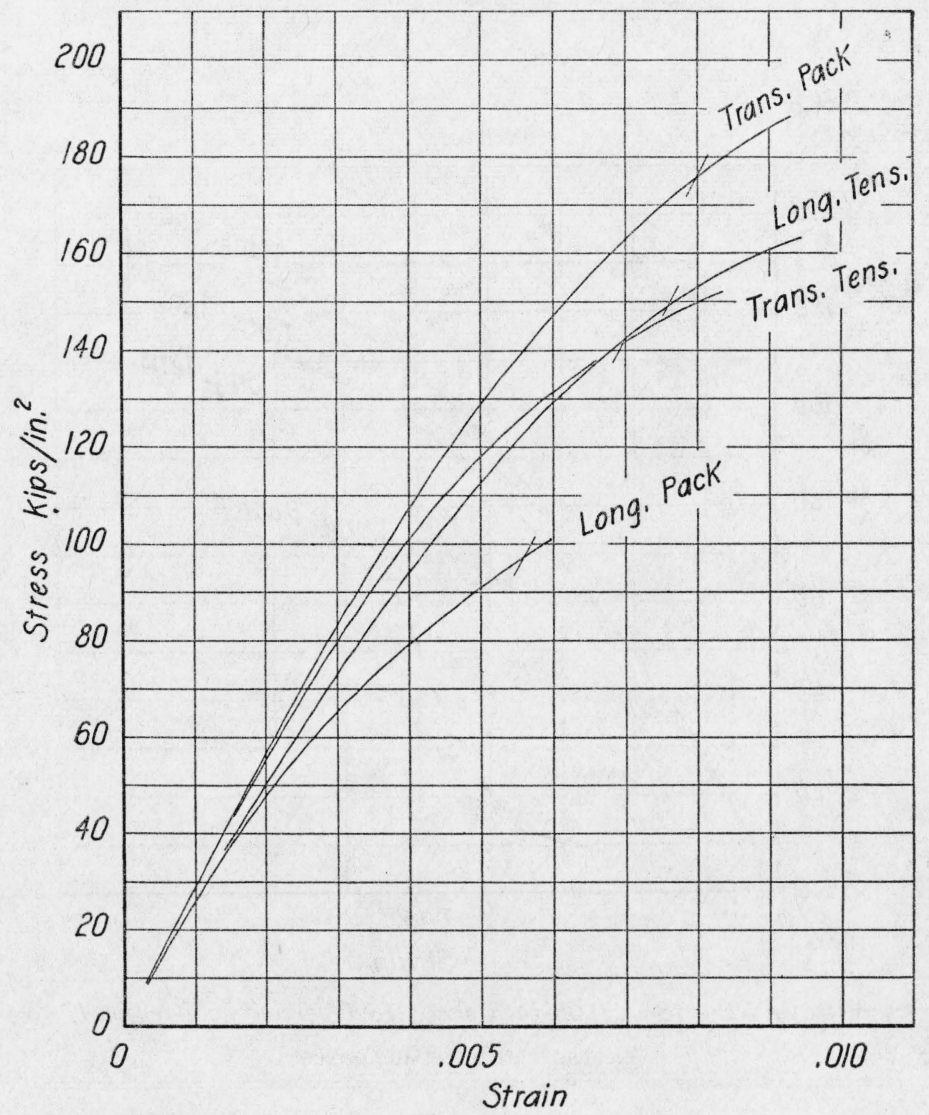

Figure 53.-18- $\gamma$, 0.06-inch sheet, 30.5 percent cold-reduced. Yield strength, offset $=0.2$ percent

\begin{tabular}{|c|c|c|}
\hline Test & Longitudinal & Transverse \\
\hline $\begin{array}{l}\text { Tensile } \\
\text { Pack compressive. }\end{array}$ & $\begin{array}{r}\text { Kips/in.2 } \\
148.6 \\
97.4\end{array}$ & $\begin{array}{r}\text { Kips/in. } .^{2} \\
141.4 \\
176.0\end{array}$ \\
\hline
\end{tabular}




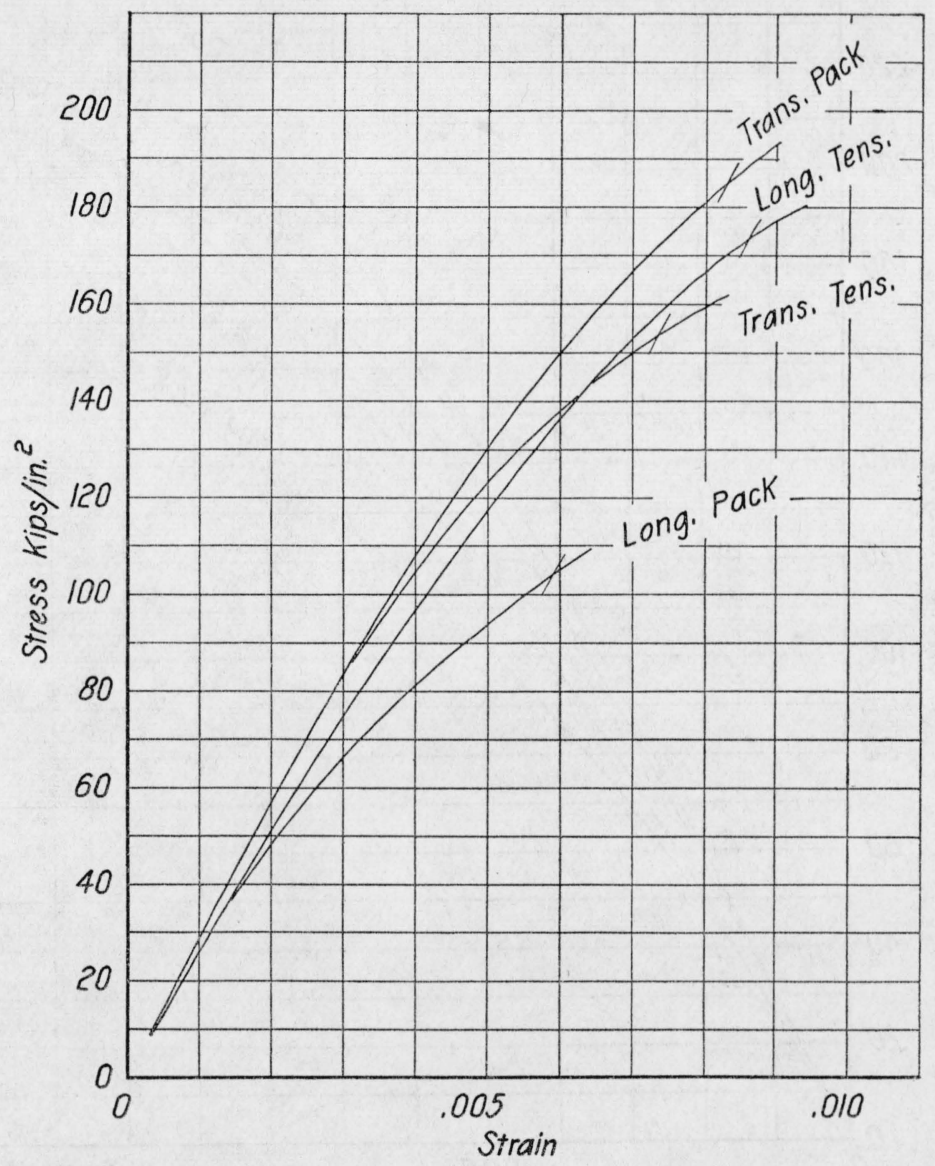

FiguRE 54.-18- $\gamma, 0.06$-inch sheet, 36.9 percent cold-reduced.

Yield strength, offiset $=0.2$ percent

\begin{tabular}{|c|c|c|}
\hline Test & Longitudinal & Transverse \\
\hline $\begin{array}{l}\text { Tensile } \\
\text { Pack compressive. }\end{array}$ & $\begin{array}{r}\text { Kips/in. } \\
173.7 \\
104.3\end{array}$ & $\begin{array}{r}\text { Kips/in. } .^{2} \\
153.8 \\
185.3\end{array}$ \\
\hline
\end{tabular}




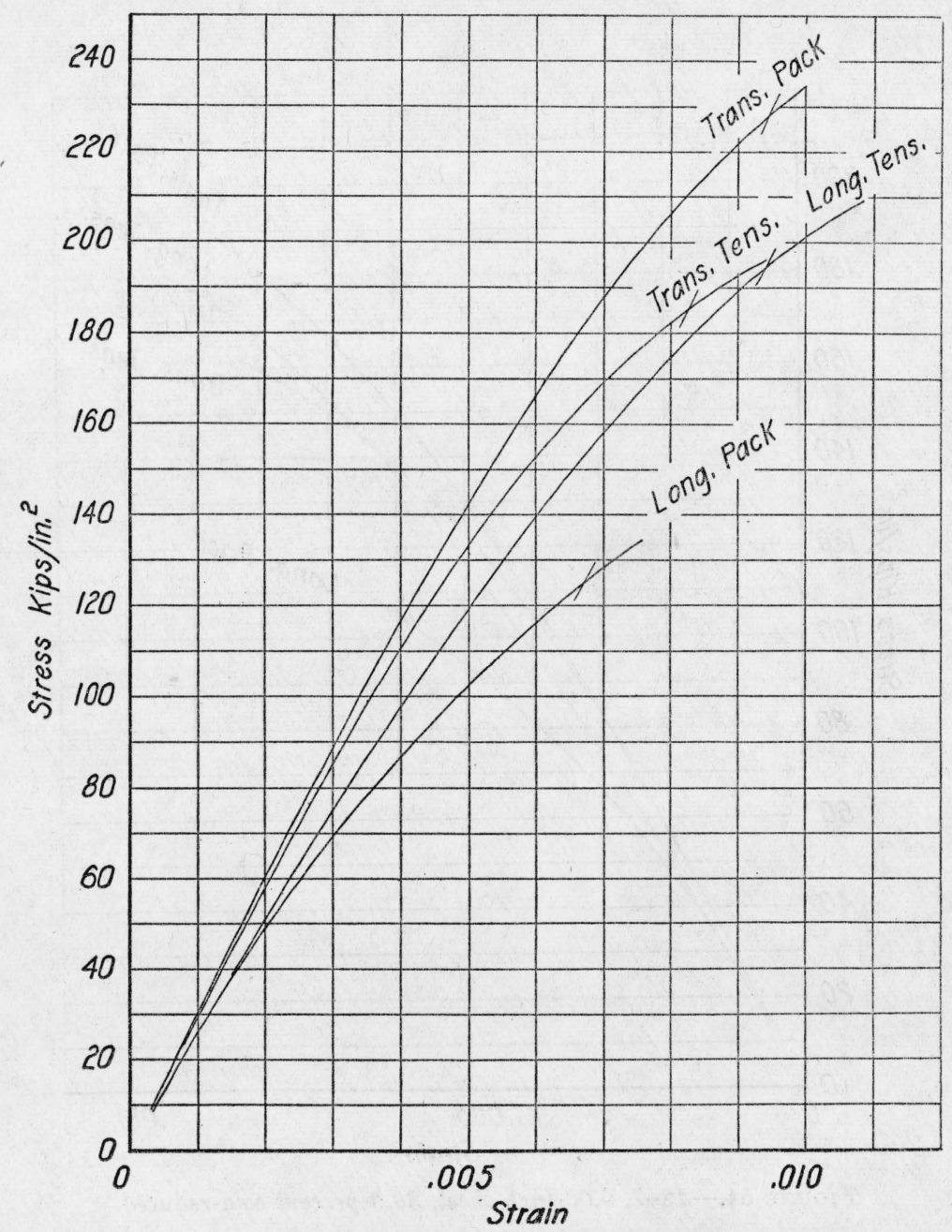

FIGURE 55.-18-7, 0.06-inch sheet, 47.7 percent cold-reduced.

Yield strength, offset $=0.2$ percent

\begin{tabular}{|c|r|r|}
\hline Test & Longitudinal & Transverse \\
\hline & Kips/in.2. \\
194.3 & $\begin{array}{r}\text { Kips/in. } .^{2} \\
185.3 \\
228.2\end{array}$ \\
\hline
\end{tabular}




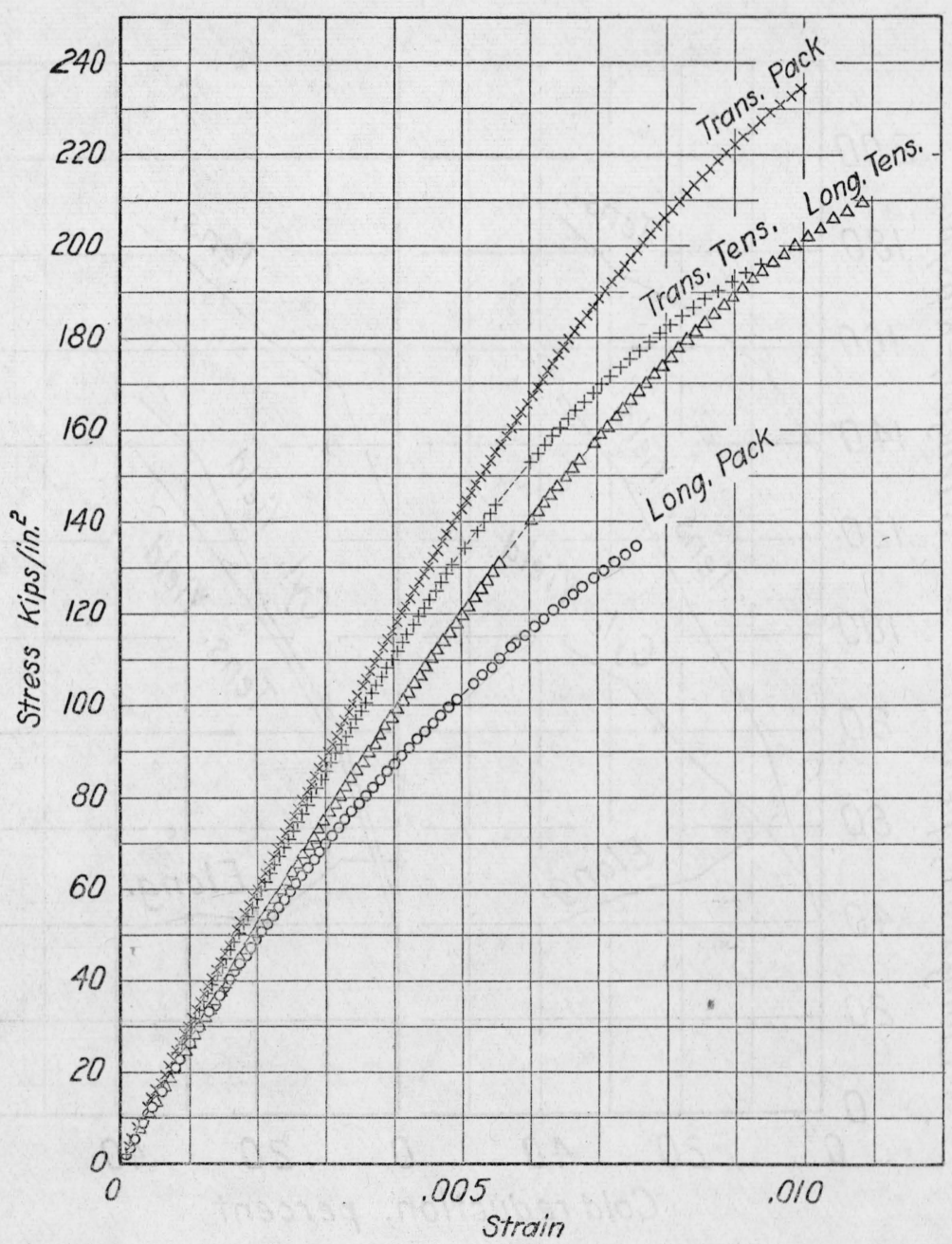

Figure 56.-18- $\gamma, 0.06$-inch sheet, 47.7 percent cold-reduced, individual points.

Both strain gages were read simultaneously at increments of about $2.0 \mathrm{kips} / \mathrm{in}^{2}$. Gaps in the readings were unavoidable, however, where occasional readings were missed or where, to avoid resetting the strain gage, both the tensile and compressive images were used. 


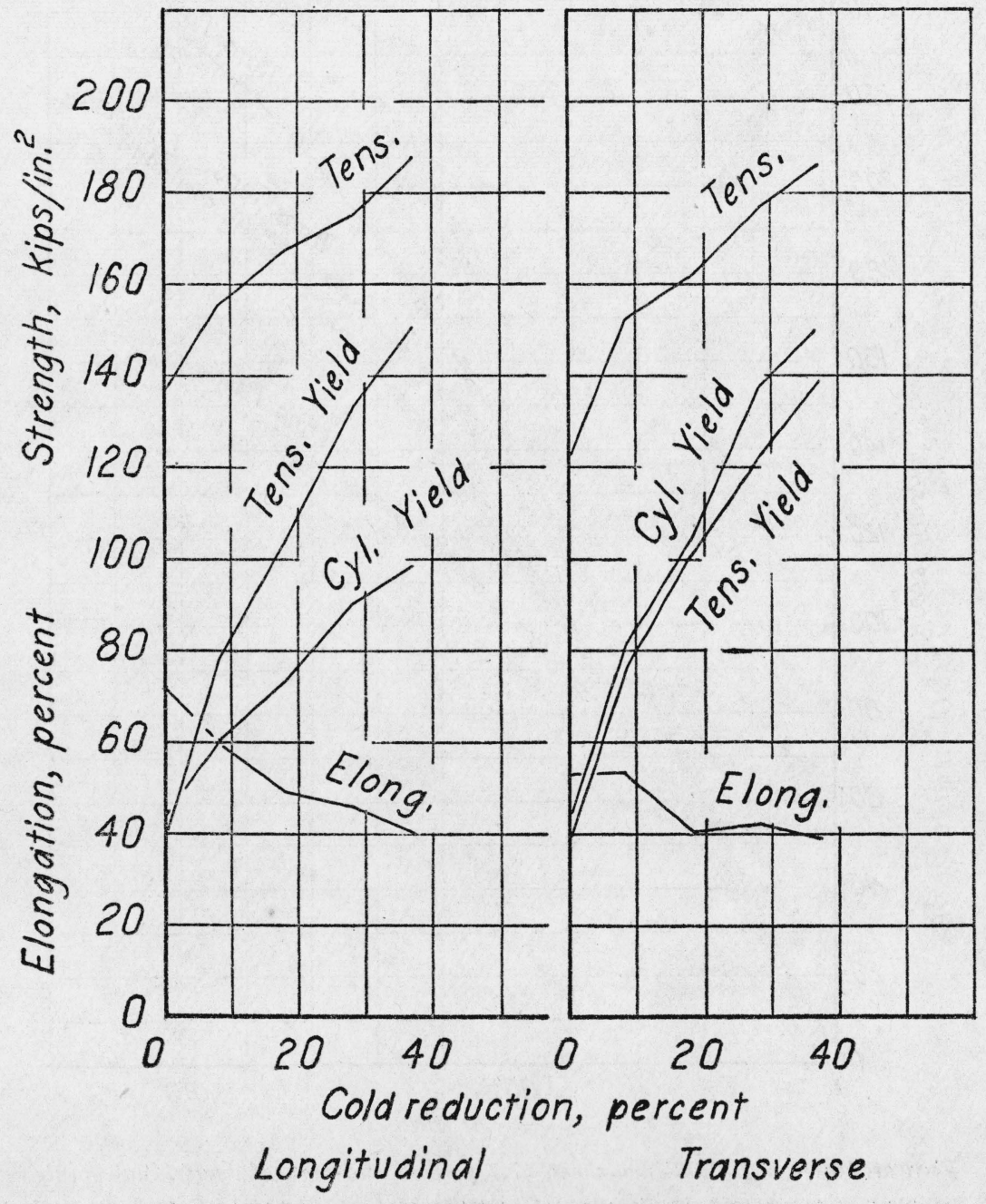

Figure 57.-17-7, 0.01-inch sheet. 


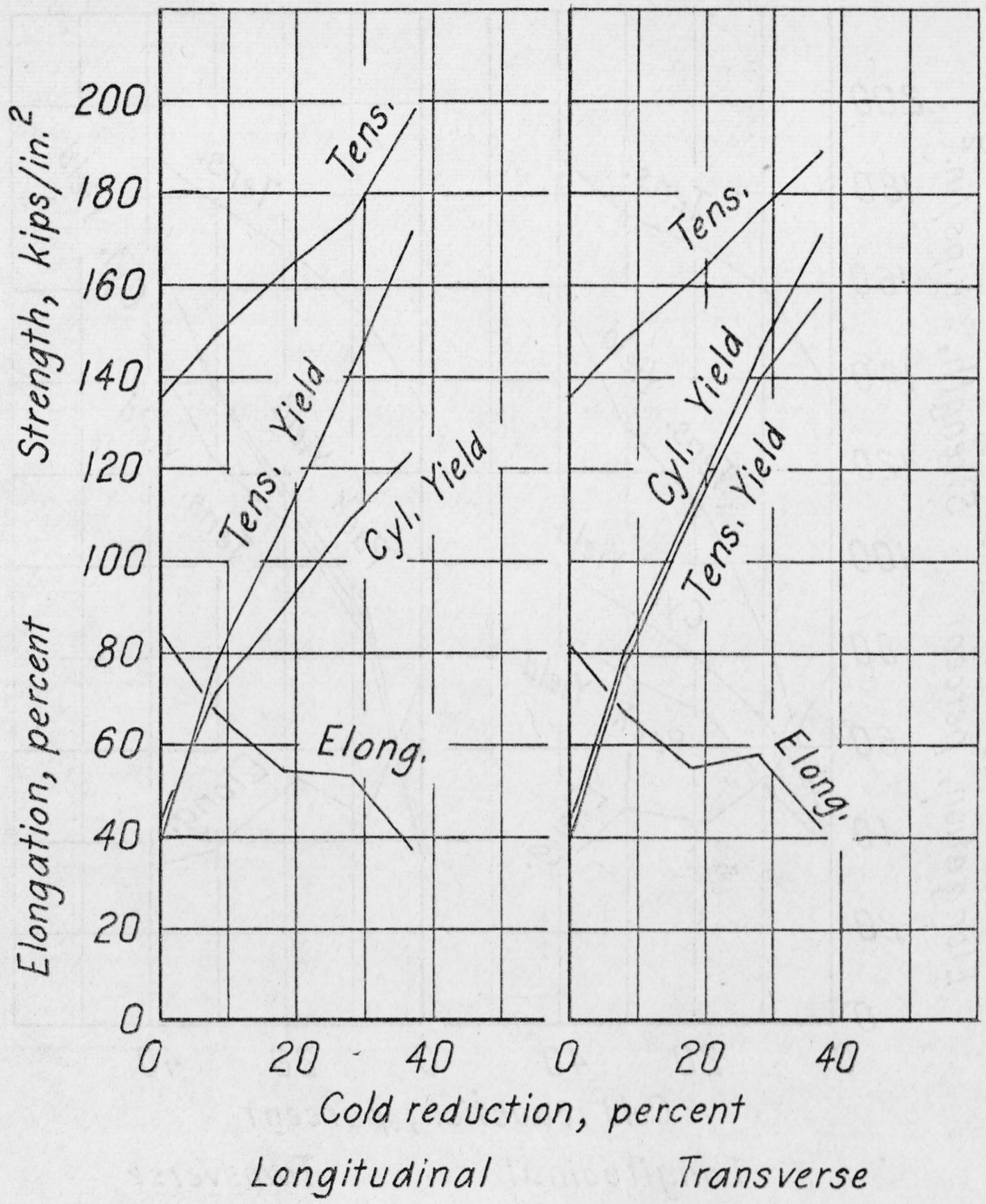

FIGURE 58.-17-7, 0.01-inch sheet, stress-relieved. 


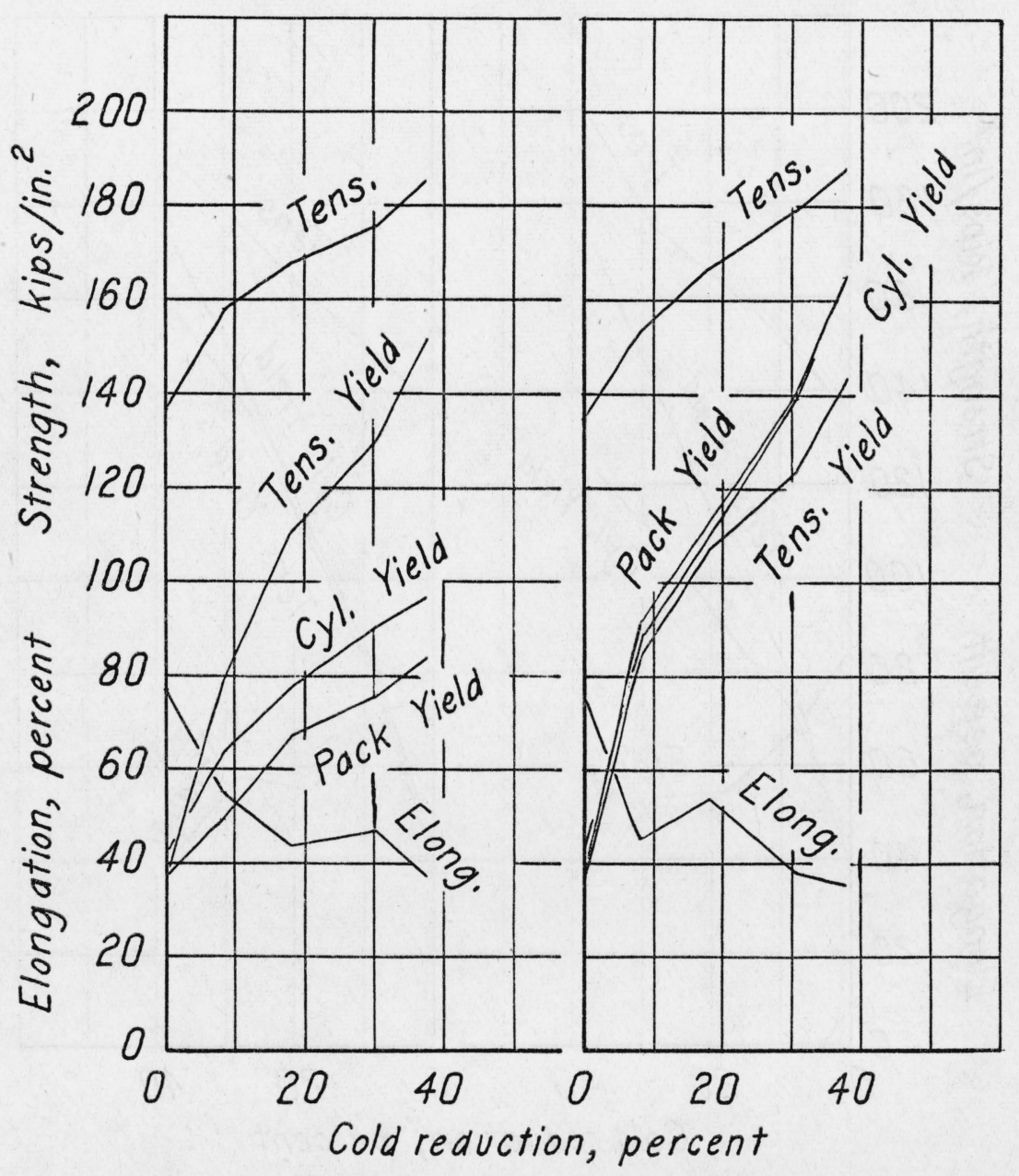

Longitudinal

Transverse

FIGURE 59.-17-7, 0.02-inch sheet. 


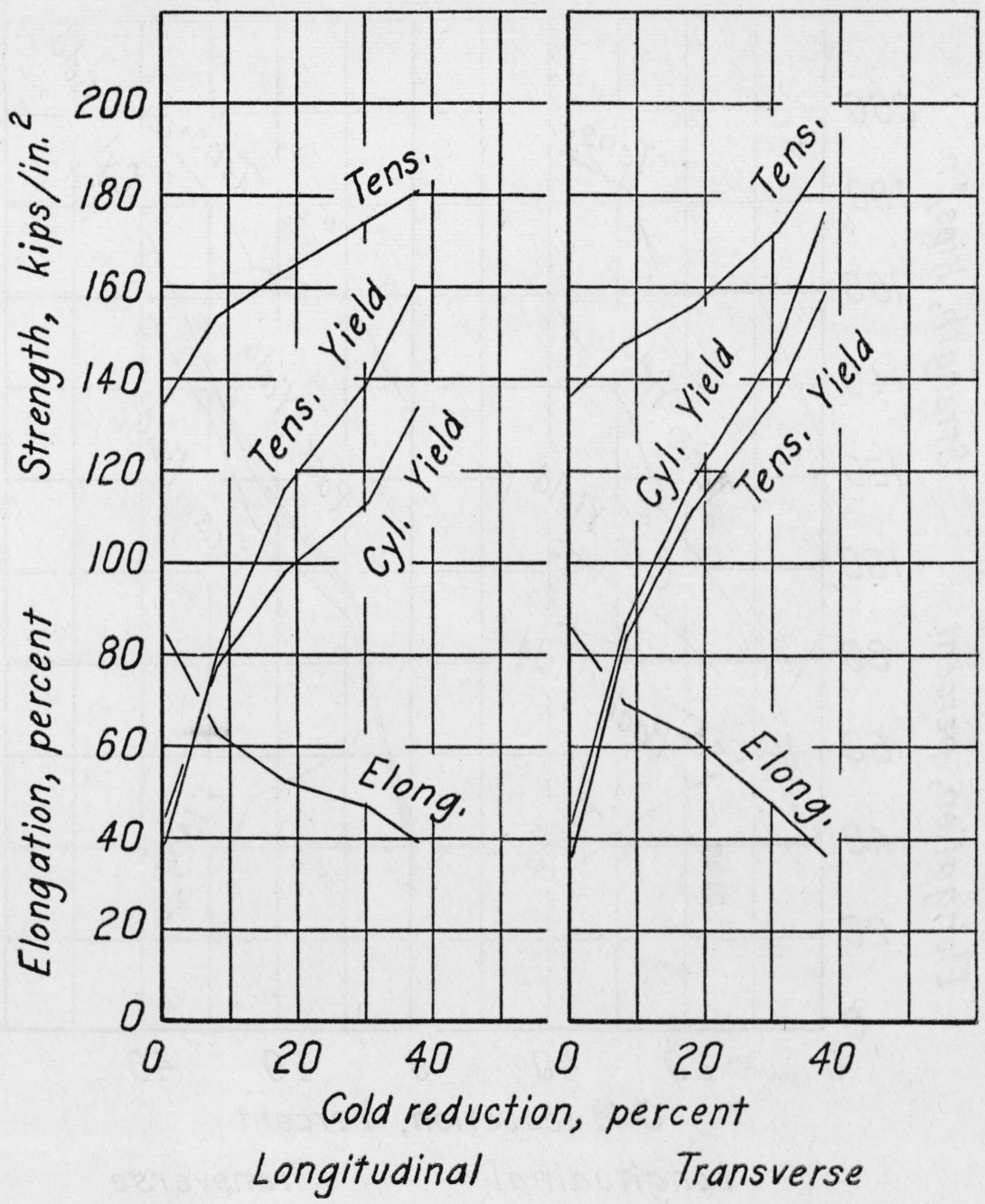

FiguRE 60.-17-7, 0.0\%-inch sheet, stress-relieved. 


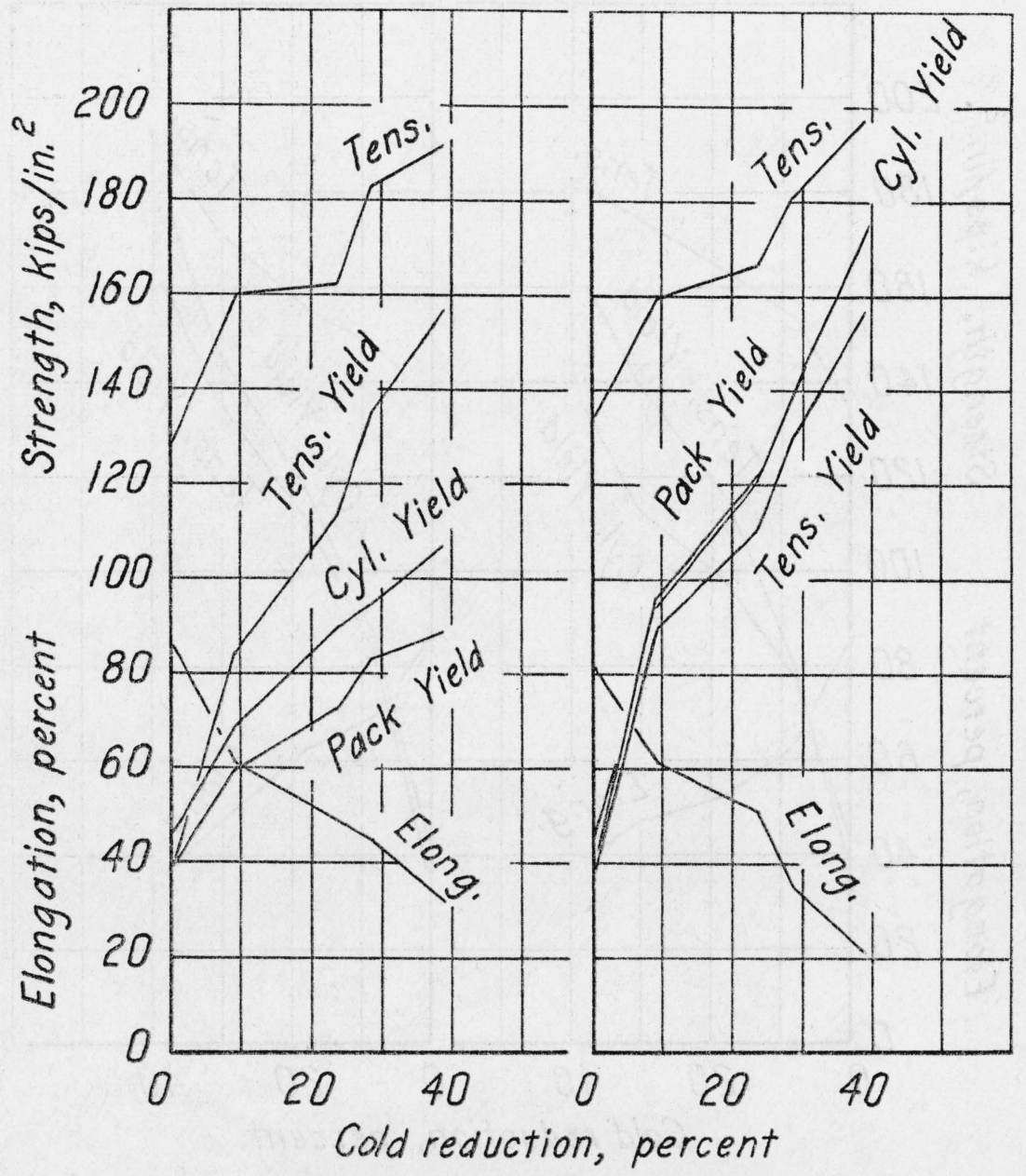
Longitudinal

Transverse

Figure $61 .-17-7,0.04-$ inch sheet. 


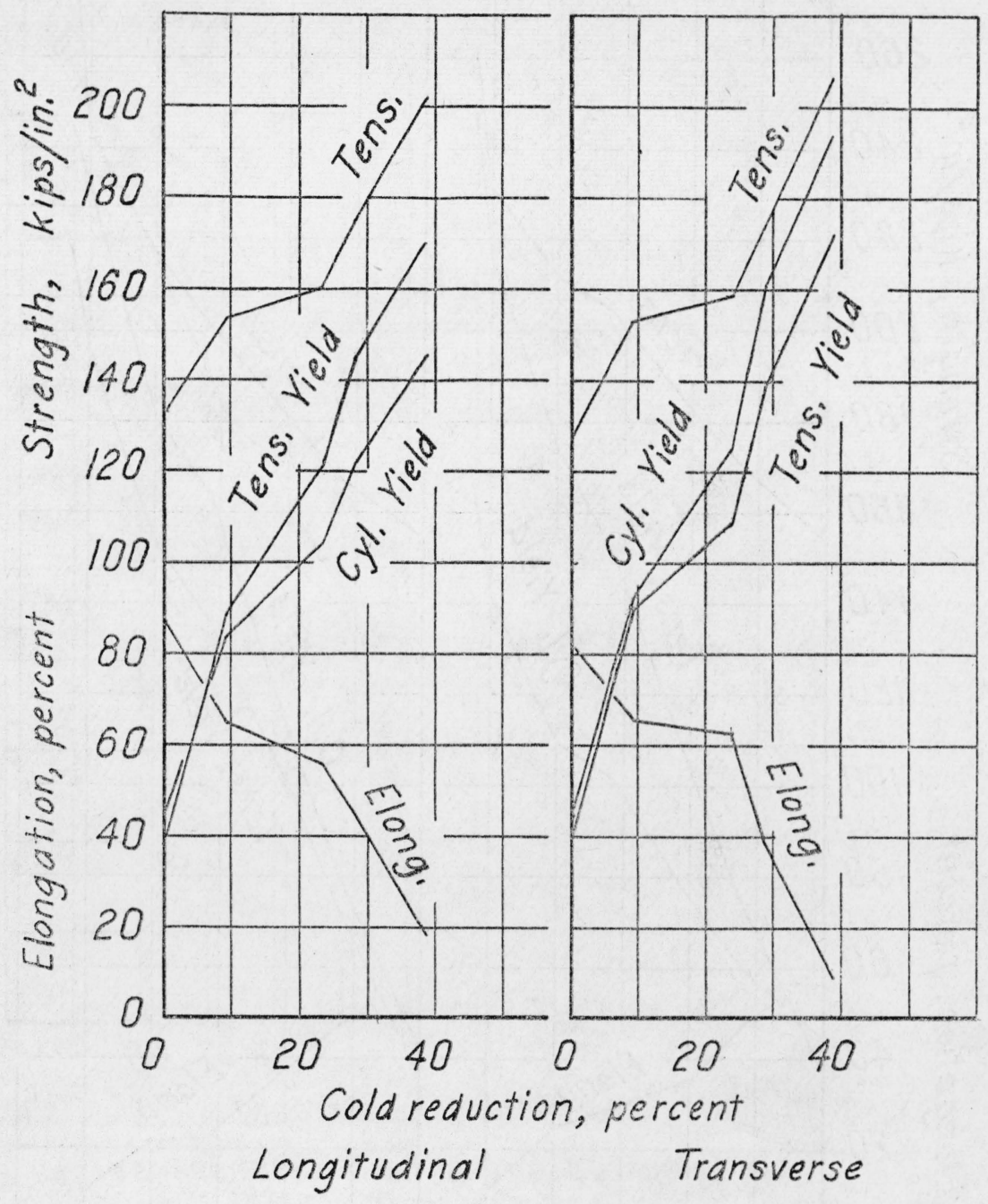

Figure 62.-1 $17-\gamma, 0.04$-inch sheet, stress-relieved. 
564 Journal of Research of the National Bureau of Standards

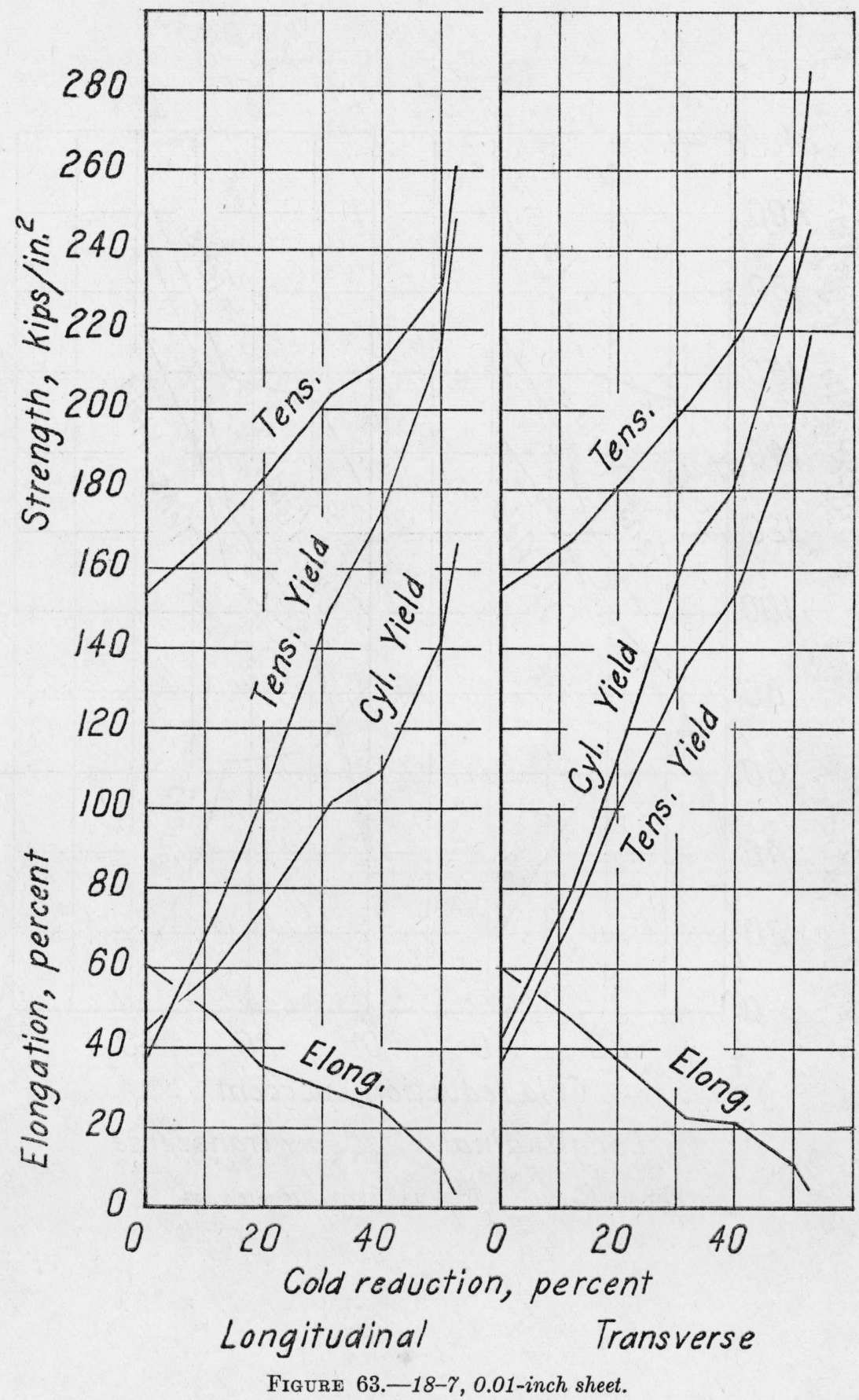




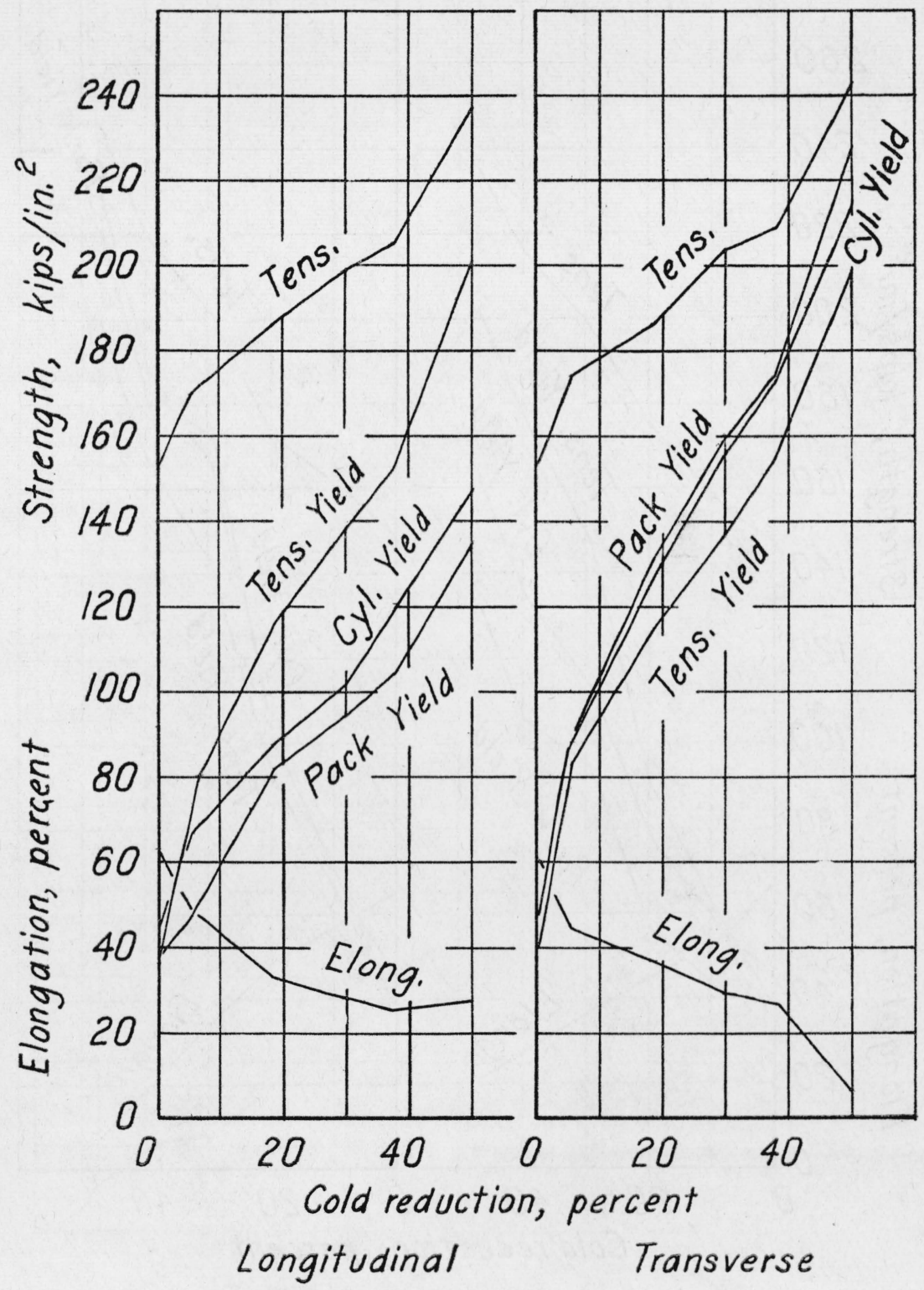

FigURE 64.-18-7, 0.02-inch sheet. 
566 Journal of Research of the National Bureau of Standards

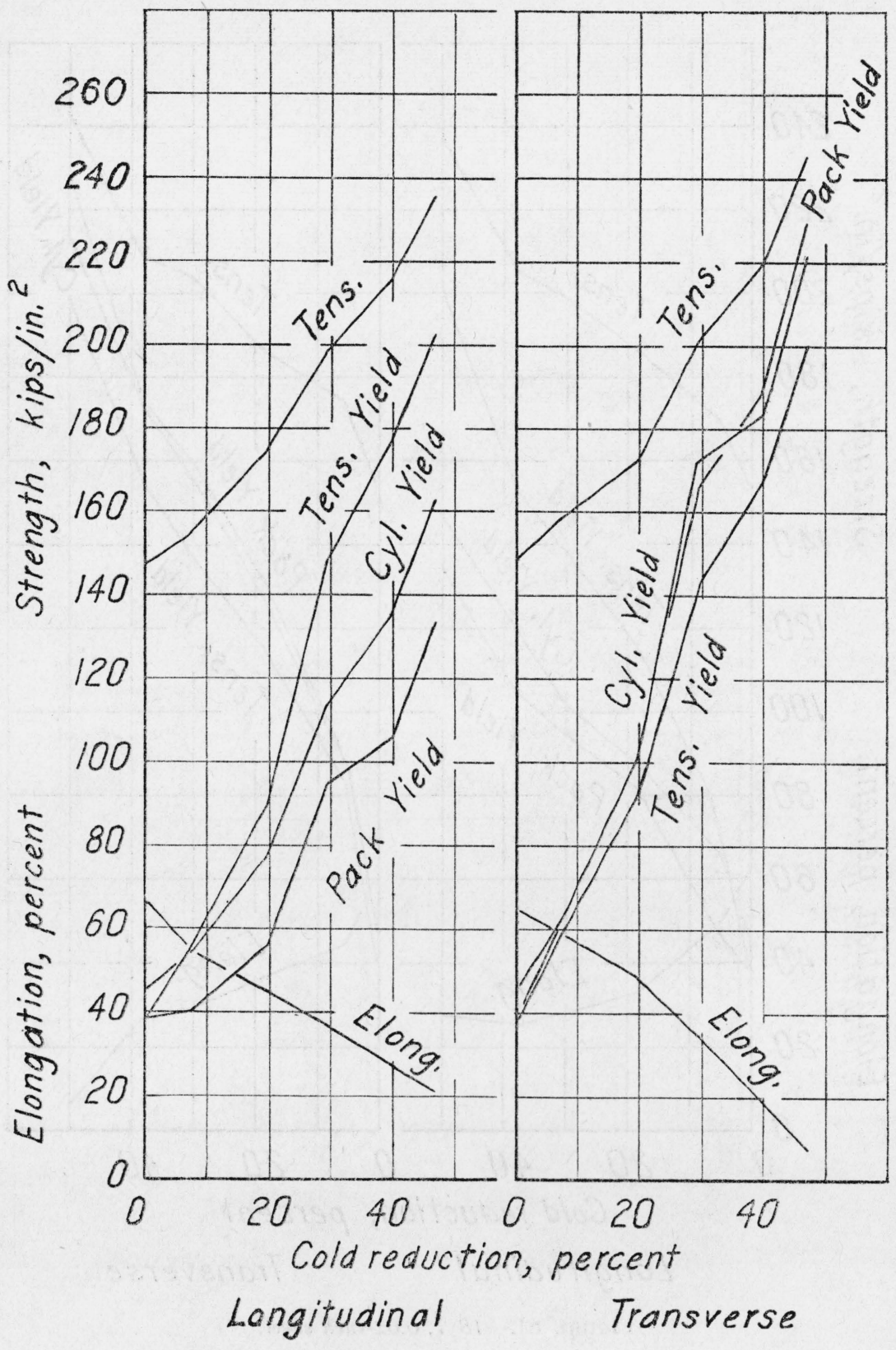

Figure 65.-18- $\gamma, 0.035$-inch sheet. 


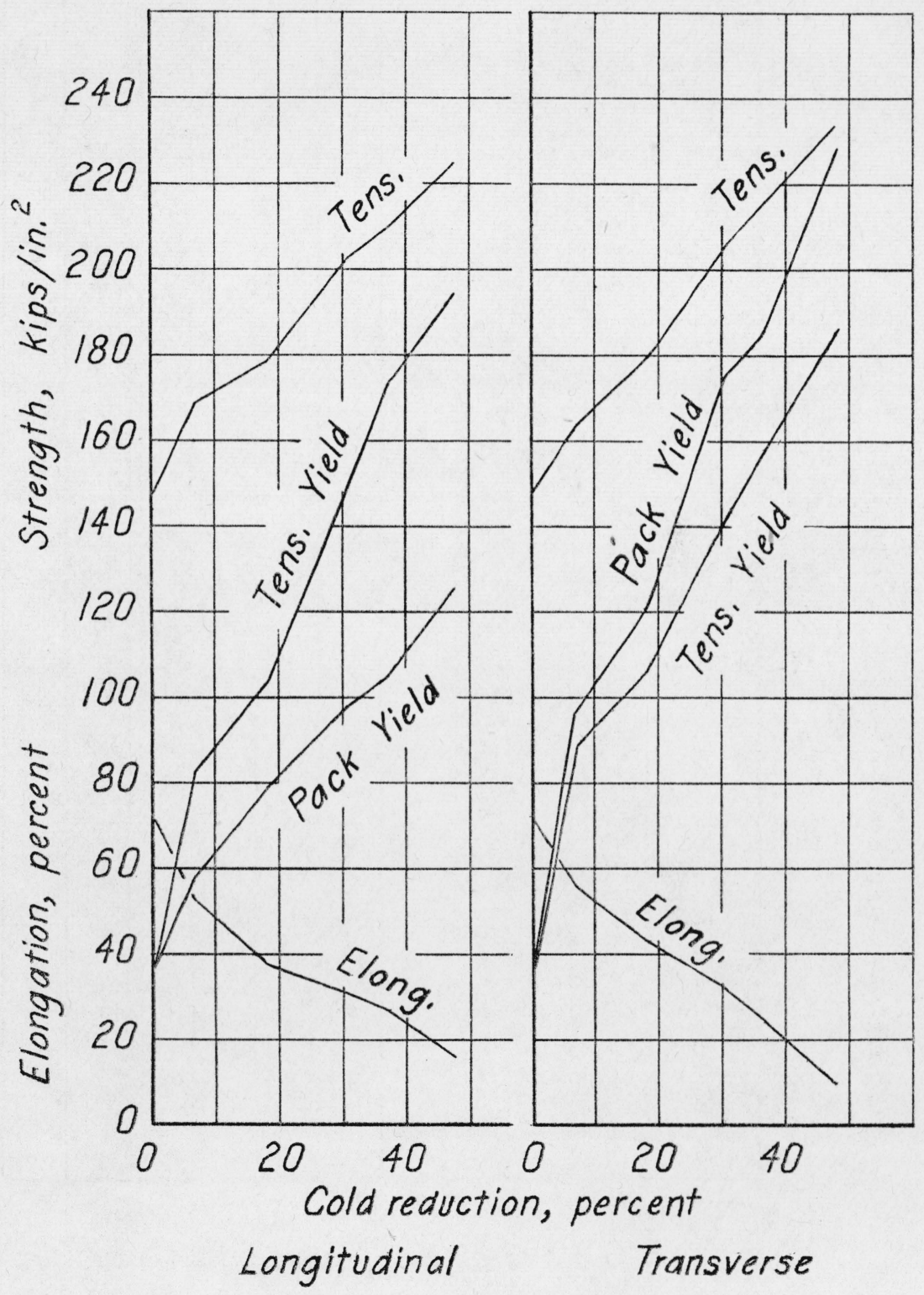

Figure 66.-18-7, 0.06-inch sheet.

Washington, March 2, 1942 


\section{NATIONAL BUREAU OF STANDARDS,}

WASHINGTON, D. C.

Send me the Mathematical Tables marked $\mathrm{X}$ below. I enclose remittance ${ }^{1}$ to cover the cost.

\begin{tabular}{|c|c|c|c|c|}
\hline \multirow[b]{2}{*}{ Mark X } & \multirow[b]{2}{*}{ Title of publication } & \multicolumn{2}{|c|}{ Price } & \multirow[b]{2}{*}{ Amount enclosed } \\
\hline & & $\begin{array}{l}\text { United States and } \\
\text { its p ossessions, } \\
\text { and countries ex- } \\
\text { tending franking } \\
\text { privilege }\end{array}$ & Other countries & \\
\hline $\begin{array}{c} \\
\end{array}$ & $\begin{array}{l}\text { MT1. Table of the first ten powers of the integers from } 1 \text { to } 1000 \\
\text { MT2. Tables of the exponential function } e^{x} \\
\text { MT3. Tables of circular and hyperbolic sines and cosines for radian arguments. } \\
\text { MT4. Tables of sines and cosines for radian arguments } \\
\text { MT5. Tables of sine, cosine, and exponential integrals, volume I } \\
\text { MT6. Tables of sine, cosine, and exponential integrals, volume } \\
\text { MT7. Table of natural logarithms, volume I } \\
\text { MT8. Tables of probability functions, volume I } \\
\text { MT9. Table of natural logarithms, volume II } \\
\text { MT10. Table of natural logarithms, volume III } \\
\text { Total remittance. }\end{array}$ & $\begin{array}{r}\$ 0.50 \\
2.00 \\
2.00 \\
2.00 \\
2.00 \\
2.00 \\
2.00 \\
2.00 \\
2.00 \\
2.00\end{array}$ & $\begin{array}{r}\$ 0.65 \\
2.50 \\
2.50 \\
2.50 \\
2.50 \\
2.50 \\
2.50 \\
2.50 \\
2.50 \\
2.50\end{array}$ & (1- \\
\hline
\end{tabular}

1 Remittance should be in form of post-office money order, or check, and made payable to the order of the "National Bureau of Standards" in United States currency.

Send to

Number and Street

City and State. 


\section{MATHEMATICAL TABLES}

Attention is invited to a series of publications which is being prepared by the Project for the Computation of Mathematical $\tau$ ables conducted by the Federal Works Agency, Work Projects Administration for the City of New York under the sponsorship of the National Bureau of Standards.

To date, ten tables have been made available through the National Bureau of Standards. These are listed below:

MT1. Table or the First Ten Powers of the Intrgers From 1 to 1000:

(1938) VIII+80 pages; heavy paper cover. 50 cents.

MT2. Tables of the Exponential Function $e^{\boldsymbol{x}}$ :

The ranges and intervals of the argument and the number of decimal places in the entries are given below:

$\begin{array}{rcc}\text { Range of } x & \text { Interval of } \boldsymbol{x} & \text { Decimals given } \\ -2.5000 \text { to } 1.0000 & 0.0001 & 18 \\ 1.0000 \text { to } 2.5000 & .0001 & 15 \\ 2.500 \text { to } 5.000 & .001 & 15 \\ 5.00 \text { to } 10.00 & .01 & 12\end{array}$

(1939) XV+535 pages; bound in buckram, $\$ 2.00$.

MT3. Tables of Circular and Hyperbolic Sings and Cosings for Radian Arguments:

Contains 9 decimal place values of $\sin x, \cos x, \sinh x$ and $\cosh x$ for $x$ (in radians) ranging from 0 to 2 at intervals of 0.0001 .

(1939) XVII + 405 pages; bound in buckram, $\$ 2.00$.

MT4. Tables of Sines and Cosines for Radian Arguments:

Contains 8 decimal place values of sines and cosines for radian arguments ranging from 0 to 25 at intervals of 0.001 .

(1940) XXIX + 275 pages; bound in buckram, $\$ 2.00$.

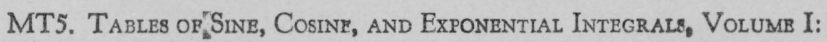

Values of these functions to 9 places of decimals from 0 to 2 at intervals of 0.0001 .

(1940) XXVI+444 pages; bound in buckram, $\$ 2.00$.

MT6. Tables of Sink, Cosine, and Exponential Integrals, Voluma IIs

Values of these functions to 9,10 , or 11 significant figures from 0 to 10 at intervals of 0.001 with auxiliary tables.

(1940) XXXVII+ 225 pages; bound in buckram, $\$ 2.00$.

MT7. Table of Natural Logarithms, Volume I:

Logarithms of the integers from 1 to 50,000 to 16 places of decimale.

(1941) XVIII + 501 pages; bound in buckram, $\$ 2.00$.

MT8 Tables of Probability Functions, Volume I:

Values of these functions to 15 places of decimals from 0 to 1 at intervals of 0.0001 and 1 to 5.6 at intervals of 0.001 .

(1941) XXVIII+302 pages; bound in buckram, $\$ 2.00$.

MT9 Table of Natural Logarithm, Volumb II:

Logarithms of the integers from 50,000 to 100,000 to 16 places of decimals.

(1941) XVIII+501 pages; bound in buckram, $\$ 2.00$.

【Continued on p. 4 of cover】 
MT10. Table of Natural Logarithms, Volume III:

(Logarithms of the decimal numbers from 0.0001 to 5.0000 , to 16 places of decimals.)

(1941) XVIII + 501 pages; bound in buckram, $\$ 2.00$.

Payment is required in advance. Make remittance payable to the "National Bureau of Standards", and send with order, using the blank form facing page 3 of the cover.

The prices are for delivery in the United States and its possessions and in countries extending the franking privilege. To other countries the price of MT1 is 65 cents and that of MT2, MT3, MT4, MT5, MT6, MT7, MT8, $\mathrm{MT}$, and MT10 is $\$ 2.50$ each; remittance to be made payable in United States currency.

Copies of these publications have been sent to various Government depositories throughout the country, such as public libraries in large cities, and colleges and universities, where they may be consulted.

A mailing list is maintained for those who desire to receive announcements regarding new tables as they become available. A list of the tables it is planned to publish will be sent on request. 